\title{
High-Resolution Microscopy with Photoswitchable Organic Markers
}

\author{
Dissertation \\ zur Erlangung des Doktorgrades \\ der Mathematisch-Naturwissenschaftlichen Fakultäten \\ der Georg-August-Universität zu Göttingen
}

\author{
vorgelegt von \\ Jonas Fölling \\ aus Oldenburg (Oldb)
}

Göttingen, den 22.10.2008 
D7

Referent: Prof. Dr. T. Salditt

Koreferent: Prof. Dr. S. W. Hell

Tag der mündlichen Prüfung: 20.11.2008 


\section{High-Resolution Microscopy with Photoswitchable Organic Markers}

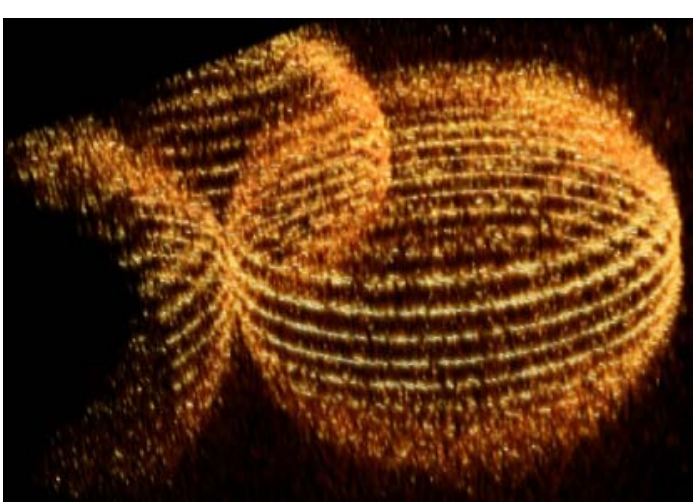





\section{Contents}

Introduction vii

1 Conventional Fluorescence Microscopy and Breaking the Diffraction Barrier 1

1.1 Introduction to Fluorescence $\ldots \ldots \ldots \ldots \ldots \ldots$

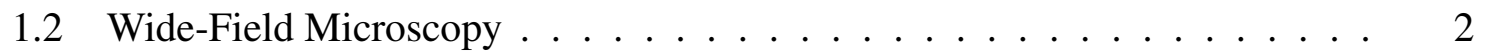

1.2 .1 Typical Microscope Setup . . . . . . . . . . . . . . . 2

1.2 .2 Point Spread Function and Image Formation . . . . . . . . . . 4

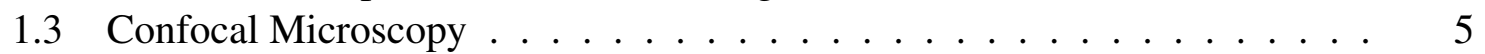

1.3 .1 Typical Microscope Setup and Imaging $\ldots \ldots \ldots \ldots$

1.3.2 Resolution of a Confocal Microscope . . . . . . . . . . . . 6

1.4 Breaking the Diffraction Barrier with RESOLFT-Microscopy . . . . . . . . 7

1.4.1 The General Principle $\ldots \ldots \ldots \ldots \ldots \ldots$

1.4 .2 Creation of Switch-off PSF by Phase Modulation . . . . . . . . 8

1.4.3 The Switch-off Kinetics and Resolution of a RESOLFT-Microscope 10

1.5 Breaking the Diffraction Barrier with SMS-Microscopy $\ldots \ldots \ldots \ldots \ldots$

1.5 .1 The General Principle $\ldots \ldots \ldots \ldots$

1.5 .2 Typical Image Acquisition . . . . . . . . . . . . . . . . . . 15

1.5 .3 Localisation and its Precision $\ldots \ldots \ldots \ldots \ldots$

1.5 .4 Photon Statistics $\ldots \ldots \ldots \ldots$

1.5 .5 Image Construction and Display . . . . . . . . . . . . . . . 19

1.5 .6 Required Marker Properties $\ldots \ldots \ldots \ldots$

1.6 Similarities and Differences of SMS and RESOLFT . . . . . . . . . . . 21

2 RESOLFT-Microscopy with Photoswitchable Organic Dyes 24

2.1 The Switching Mechanism . . . . . . . . . . . . . . . . . 24

2.2 RESOLFT-Microscopy with a Mixture of Photochromic and Fluorescent Dyes 26

2.2.1 Properties and Kinetics of the Photochrome-Fluorophore System. . 26

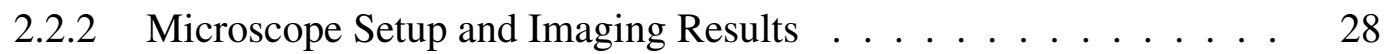

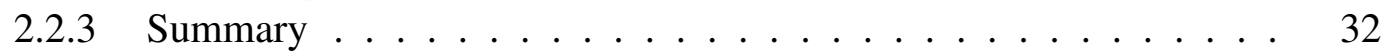

2.3 Photoswitching and Imaging of Organic Fluorescent Switches in Silica Nanopar-

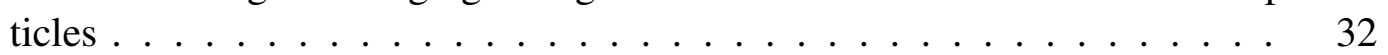

2.3.1 Properties of the Covalently Bound Photochrome-Fluorophore System 32

2.3 .2 Application in RESOLFT-Microscopy $\ldots \ldots \ldots \ldots$. . . . . . . . 35

2.3 .3 Application as Switchable Tracer $\ldots \ldots \ldots$. . . . . . . . . . . . 39

2.3 .4 Summary $\ldots \ldots \ldots \ldots \ldots \ldots \ldots \ldots \ldots \ldots \ldots$ 
$3.1 \quad$ SMS-Microscopy With Optical Sectioning Using Two-Photon Activation . 41

3.1.1 The Novel Family of Photoswitchable Compounds . . . . . . . . . 42

3.1.2 Optical Sectioning with Two-Photon Activation . . . . . . . . . . . 43

3.1.3 The Switchable Rhodamine Amide SRA577 . . . . . . . . . . . . 44

3.1 .4 Experimental Setup . . . . . . . . . . . . . . . . . . . 45

3.1 .5 Experimental Results . . . . . . . . . . . . . . . . . . . . . . . . . . . . . . . . . .

3.1 .6 Two-Photon Activation with cw-lasers . . . . . . . . . . . . . 50

3.1 .7 Summary . . . . . . . . . . . . . . . . 53

3.2 Multicolour SMS-Imaging by Single Molecule Spectroscopy . . . . . . . . 54

3.2.1 Colour Separation Principle . . . . . . . . . . . . . 55

3.2 .2 Markers . . . . . . . . . . . . . . . . . 60

$3.2 .3 \quad$ Imaging Setup, Registration and Type Assignment . . . . . . . . . 61

$3.2 .4 \quad$ Experimental Results . . . . . . . . . . . . . . . . . 63

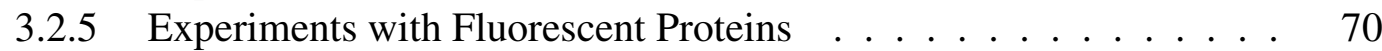

3.2 .6 Summary . . . . . . . . . . . . . . . 73

3.3 SMS-Imaging with Conventional Markers using Ground State Depletion . . 74

3.3.1 Ground State Depletion. . . . . . . . . . . . . . . . . 74

3.3 .2 Staining Density $\ldots \ldots \ldots \ldots$. . . . . . . . . . . . . . . . . . . . . . . . . . . . .

3.3 .3 Imaging and Results $\ldots \ldots \ldots$. . . . . . . . . . . . . . . . . . . . . . . . .

3.3 .4 Summary $\ldots \ldots \ldots \ldots$. . . . . . . . . . . . . . 85

$\begin{array}{lll}4 & \text { Conclusion } & 86\end{array}$

\begin{tabular}{ll}
\hline Bibliography & 89
\end{tabular}

\begin{tabular}{ll}
\hline A Appendix & 97
\end{tabular}

A.1 Standard Cell Preparation . . . . . . . . . . . . . . . . . . . . . . . . . . . 97

A.2 Immunostaining of Cells . . . . . . . . . . . . . . . . . . . . . . . . . . 97

A.3 Staining with Fluorescent Proteins . . . . . . . . . . . . . . . . . . 98

\begin{tabular}{ll}
\hline B List of Publications & 100
\end{tabular}

\begin{tabular}{ll}
\hline Acknowledgement & 101
\end{tabular} 


\section{Introduction}

Light microscopy is one of the most powerful imaging tools in the life sciences and beyond. Its versatility is its major advantage. Manifold staining techniques allow one to tag the areas and structures of interest with fluorescent markers which give a formidable signal to noise ratio. Different structures can be stained with dyes of distinguishable properties, such as different emission spectra. Therefore the co-localisation and interaction of cellular structures or molecules and the temporal development of the involved mechanisms can be easily followed. Markers sensitive to their environment can be used to observe cellular processes such as changes in membrane potentials or ion exchange via the cell membrane. This includes imaging in living cells, since light microscopy is non-invasive and non-destructive and compatible with live-cell environments. In its far-field variants, light microscopy is further capable of imaging in thick media such as whole cells or even larger pieces of tissue. Mechanical slicing of fixed objects is thus not mandatory. These advantages make light microscopy indispensable despite its major drawback: All conventional techniques suffer from the diffraction limit stated by Ernst Abbe in 1873 which represents a boundary for the spatial resolution of the microscope [1]. Any point-like object will not be imaged as a point but as an extended circular spot. A more complex object containing structures smaller than the resolution appears blurred in the image and the structures cannot be revealed. Abbe stated this spatial resolution limit, $\Delta r$, to be

$$
\Delta r=0.5 \frac{\lambda}{n \cdot \sin \alpha}
$$

in the lateral directions. Here, $n$ is the refractive index of the embedding medium of the object, $\alpha$ is the semiaperture angle of the objective lens and $\lambda$ the wavelength of the light. This distance cannot be reduced arbitrarily. Increasing the value of $n \cdot \sin \alpha$, which is the so called 'numerical aperture', is delicate. Typical contemporary objectives feature a value of $n \cdot \sin \alpha \approx 1$.4. Using visible light or near-UV light, the resolution limit is thus reached at about $200 \mathrm{~nm}$. Reducing $\lambda$ further into the UV range is hardly compatible with living cells due to the photodamage induced by the high-energy photons. Furthermore, conventional lens-based optics cannot be applied any more, thus extremely costly objectives have to be applied, using mirrors instead of lenses.

The invention of electron microscopy opened up a successful alternative. The de Broglie wavelength of electrons is much smaller than the wavelength of visible light and resolutions below $1 \mathrm{~nm}$ are possible. Electron microscopy, however, suffers from the fact that it is not live-cell compatible since it requires vacuum conditions and induces sample damage due to the high energy of the electrons. The sample preparation is complex as only thin samples can be imaged, thus (cryo-)slicing is mandatory if the interior of thick samples is to be investigated. Additionally, labelling the areas of interest is by far not as flexible as in light 
microscopy.

Atomic force microscopes [2] can image surfaces with extremely high accuracy, but cannot penetrate into samples. This also applies to near field light microscopy techniques using ultrasharp tips or tiny apertures [3] and the relatively new field of negative refractive index lenses [4, 5, 6], which require close contact of the sample to the imaging device.

For these reasons, much effort has been undertaken to further push the diffraction barrier of far-field light microscopes to its absolute limits with techniques such as 4 Pi microscopy [7], enabling higher resolution in the axial direction, or structured illumination microscopy [8]. However, these methods only lower the barrier but are still subject to Abbe's law.

Up to now the only way around the diffraction barrier is by going beyond using the fluorescence signal for mere detection of the markers [9]. Further inherent properties of the dyes have to be exploited, allowing one to switch them between a bright detectable state and a dark non-detectable state [10, 11]. The first method to make use of this and to fundamentally break the diffraction barrier, enabling in principle unlimited resolution, is STED-microscopy (STimulated Emission Depletion) [12]. It is a member of the family of RESOLFT-techniques (REversible Saturable OpticaL (Fluorescence) Transitions) [13]. Not long ago, another technique evolved, using stochastic single molecule switching, named PALM [14], STORM [15], fPALM [16] or PALMIRA [17, 18]. This concept will be called by the umbrella term '(stochastic) Single Marker Switching'-microscopy (SMS-microscopy) in this thesis. Both concepts, RESOLFT and SMS, are based on the same key principle to break the diffraction barrier. They use a switching transition of the marker molecules between a dark state and a bright state in order to assure that the fluorescence signal stems only from an isolated spot much smaller than the size of the diffraction limit. This way, object patterns can be resolved which are smaller than the diffraction limit. Using sequential readout and the information that the signal stems from a subdiffraction-sized region, an image of the object with a resolution much higher than that of a conventional microscope is obtained [11].

The mandatory transition between a bright and a dark state of fluorescent dyes can be achieved in different ways for the different high-resolution methods. Some RESOLFTapproaches, such as STED and Ground State Depletion (GSD) microscopy [19, 20], utilise the inherent electronic states of standard fluorophores for the switching process [12, 19]. Other RESOLFT-approaches [13] as well as hitherto all realisations of SMS-microscopy use 'really' photoswitchable markers. These are characterised by two different states which for example show different conformations, where only one of these states can fluoresce. The conformational change is induced by the absorption of a photon in order to provide the necessary energy.

Within this thesis, both concepts, RESOLFT- and SMS-microscopy, were explored and further developed using different kinds of novel photoswitchable organic dyes as well as a novel mode of application of standard organic fluorophores.

In the case of RESOLFT-microscopy, photoswitchable dyes have the advantage that low light intensities are sufficient for the switching process and thus for breaking the diffraction barrier [13, 21, 22]. Although STED microscopy is very successful and renders stunning high-resolution images, it necessitates high light intensities which may induce photodamage especially in live-cell experiments. It is therefore worthwhile to explore alternatives to 
STED. It has already been shown experimentally that photoswitchable fluorescent proteins can be used to break the diffraction barrier in the RESOLFT-approach using only low light intensities [13]. In this thesis, synthetic organic dyes are applied, which have the advantage that they are usually brighter than fluorescent proteins and can more easily be altered and adapted to specific needs.

In the case of SMS-microscopy, hitherto all SMS-experiments were based on photoswitchable markers, most of them relied on photoswitchable proteins [14, 16, 17, 18, 23]. For SMS-microscopy, a higher marker brightness directly yields a better resolution. Therefore, it is desirable to utilise the superior brightness of organic dyes. Although bright photoswitchable organic cyanine dye pairs were already applied for SMS-microscopy [15, 24], these dyes only work under special environmental conditions. A novel family of photoswitchable organic dyes is introduced in this thesis. These markers are easy to use and, furthermore, open up new possibilities for SMS-microscopy such as imaging in thick samples using optical sectioning with two-photon-activation, which is demonstrated in this thesis [25, 26].

These dyes are also used to show a novel and conceptually simple way of multicolour SMS-imaging [27, 28], which efficiently exploits the single molecule approach of SMSmicroscopy also for colour distinction. This makes it possible to clearly distinguish even dyes whose fluorescence spectra overlap significantly.

Apart from specialised photoswitchable dyes, however, also the inherent dark states of standard fluorophores can be exploited for the switching. This method is called 'Ground State Depletion' and has already been applied to RESOLFT-microscopy [19, 20]. Ground-statedepletion was successfully introduced to SMS-microscopy in the framework of this thesis [29] and represents a simple method to achieve resolution beyond the diffraction limit with a wide range of standard dyes, even in living cells.

This thesis is structured as follows. Subsequent to this introduction, Chapter 1 gives an overview of the two major conventional light microscopy techniques on which the two superresolution concepts, as they are applied here, are based. These are camera-based wide-field and confocal scanning microscopes. In this context, the resolution problem will be outlined in more detail. Subsequently, the two concepts of breaking the diffraction barrier relevant for this thesis are introduced: RESOLFT-type high resolution scanning microscopy as well as the more recently developed wide-field SMS-microscopy technique. The chapter concludes with a discussion of the major aspects of the two methods.

Chapter 2 describes the novel work on RESOLFT-microscopy performed within the framework of this thesis. The working principle of using organic switchable dyes to break the diffraction barrier, and the results obtained are presented.

Chapter 3 illustrates the research performed on SMS-microscopy. The advances using the new family of markers are presented, including the optical sectioning capabilities, as well as multicolour applications with a novel marker-distinction method. An extension of SMSmicroscopy to standard fluorescent markers is furthermore introduced.

The thesis is concluded in chapter 4 



\section{Conventional Fluorescence Microscopy and Breaking the Diffraction Barrier}

\subsection{Introduction to Fluorescence}

Modern light microscopy relies on fluorescent markers, since they provide an excellent signal to noise ratio and are available with a wide range of properties, enabling differentiated marking of the areas of interest of the object. The two concepts of superresolution microscopy which are the subject of research in this thesis also rely on fluorescent dyes and exploit their inherent properties. Therefore a general short overview of the fluorescence process will be given in this section [30, 31].

The electronic and vibrational states of a fluorescent dye can be sketched nicely in a Jablonski diagram such as in figure 1.1(a), which shows the main states of an average fluorophore. When the molecule is in the electronic ground state $S_{0}$, the electrons are paired, therefore it is a singlet state. The absolute ground state is shown in a black line, the vibrational substates are marked as grey lines. When absorbing a photon of sufficient energy, the molecule is transferred to its first excited electronic state $S_{1}$. The molecules are mainly excited to higher vibrational sub-states of $S_{1}$. They relax to the vibrational ground state of $S_{1}$ within picoseconds and without radiation, which is illustrated by the wiggly line. From here, either radiationless internal conversion (ic) to $S_{0}$ may occur or the molecule emits its energy in

(a)

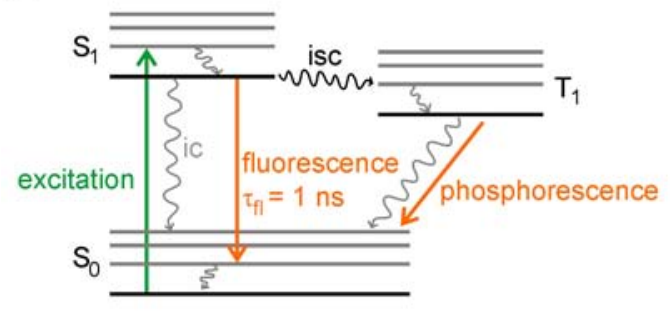

(b)

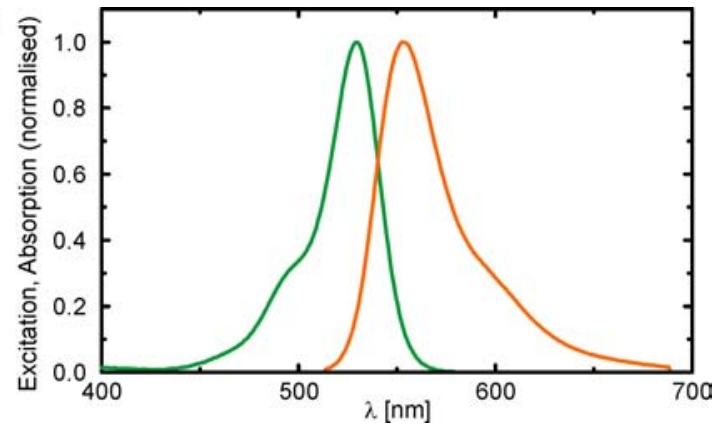

Figure 1.1: (a) Jablonski diagram of the electronic states of a fluorescent dye. $S_{0}$ and $S_{1}$ are the electronic ground state and excited state, respectively. $\mathrm{T}_{1}$ is the first excited triplet state. isc: intersystem crossing, ic: internal conversion, $\tau_{f l}$ : fluorescence lifetime. (b) Normalised excitation (green) and emission (orange) spectra of Rhodamine $6 \mathrm{G}$ in ethanol. 
the form of a photon and thus fluoresces. The lifetime $\tau_{f l}$ of the $\mathrm{S}_{1}$ state is on the order of $1 \mathrm{~ns}$ for conventional fluorophores. Due to the preceding relaxation within $\mathrm{S}_{1}$ and the likely transfer to a vibrationally excited state of $S_{0}$, the emitted photon has a longer wavelength than the exciting photon, thus leading to a redshift of the emission spectrum as compared to the excitation spectrum. This is referred to as 'Stokes-shift' and can be seen in figure 1.1(b) for the exemplary spectrum of the dye Rhodamine 6G. The excitation spectrum is plotted in green and the emission spectrum, which is in the orange spectral range, in orange. The Stokes-shift can be exploited experimentally to separate the emitted light from the excitation light and thus to obtain a detection signal with very low background noise. The excitation and emission spectra are not sharp lines but broader spectral areas, since excitation to the sub-states of $S_{1}$ is possible as well as emission to the sub-states of $S_{0}$.

Apart from the transition to $S_{0}$, however, there is a certain probability for the molecule to undergo intersystem crossing (isc), that is for the transition from the first excited electronic singlet state $S_{1}$ to the lowest excited electronic triplet state $T_{1}$. Triplet states are characterised by two unpaired electron spins showing a non-zero total angular momentum, therefore a spin-flip is necessary for a transition from a singlet to a triplet state, making it formally 'forbidden'. Strictly speaking, however, not the angular momentum $\mathbf{S}$ of the electron spins has to be conserved but the total angular momentum, $\mathbf{J}=\mathbf{L}+\mathbf{S}$, with $\mathbf{L}$ denoting the orbital angular momentum. Due to the weak coupling of the two, one can assume that the singlet wave function also has a small admixture of a triplet wave function and vice versa [30]. Therefore these 'forbidden' processes may still happen but with a very low probability. The direct transition from $S_{0}$ to $T_{1}$ is thus optically suppressed, leaving the main pathway via $S_{1}$ and subsequent intersystem crossing. The direct back-transition from $T_{1}$ to $S_{0}$, however, is also suppressed, making $\mathrm{T}_{1}$ a relatively long lived state, with a lifetime of $\tau=10^{-3}-100 \mathrm{~ms}$ depending on the dye and the environment [19]. This fact is exploited in GSD-microscopy, also in the framework of this thesis in section 3.3 . When the relaxation from $\mathrm{T}_{1}$ to $\mathrm{S}_{0}$ happens, it is most likely non-radiative. Radiative transition is referred to as 'phosphorescence' but mostly only occurs at low temperatures where the vibrational relaxation is suppressed.

\subsection{Wide-Field Microscopy}

\subsubsection{Typical Microscope Setup}

The microscopy method introduced in the following is called 'wide-field microscopy' within this thesis, it is otherwise also referred to as 'epifluorescence microscopy'. A typical microscope setup of that kind for fluorescent marker dyes is sketched in figure 1.2. The image shows a cell as a sample object, which is mounted on a glass cover slip. The object is typically illuminated through the cover slip. The region of interest is placed in the focal plane (FP, black dashed line) of the objective (OBJ) and is homogeneously illuminated by the excitation light, hence the name 'wide-field microscopy'. This can for example be easily achieved by focussing a beam of the excitation light into the back focal plane of the objective lens (BFP, black dashed line). Thus a collimated beam is shining onto the sample. The fluo- 


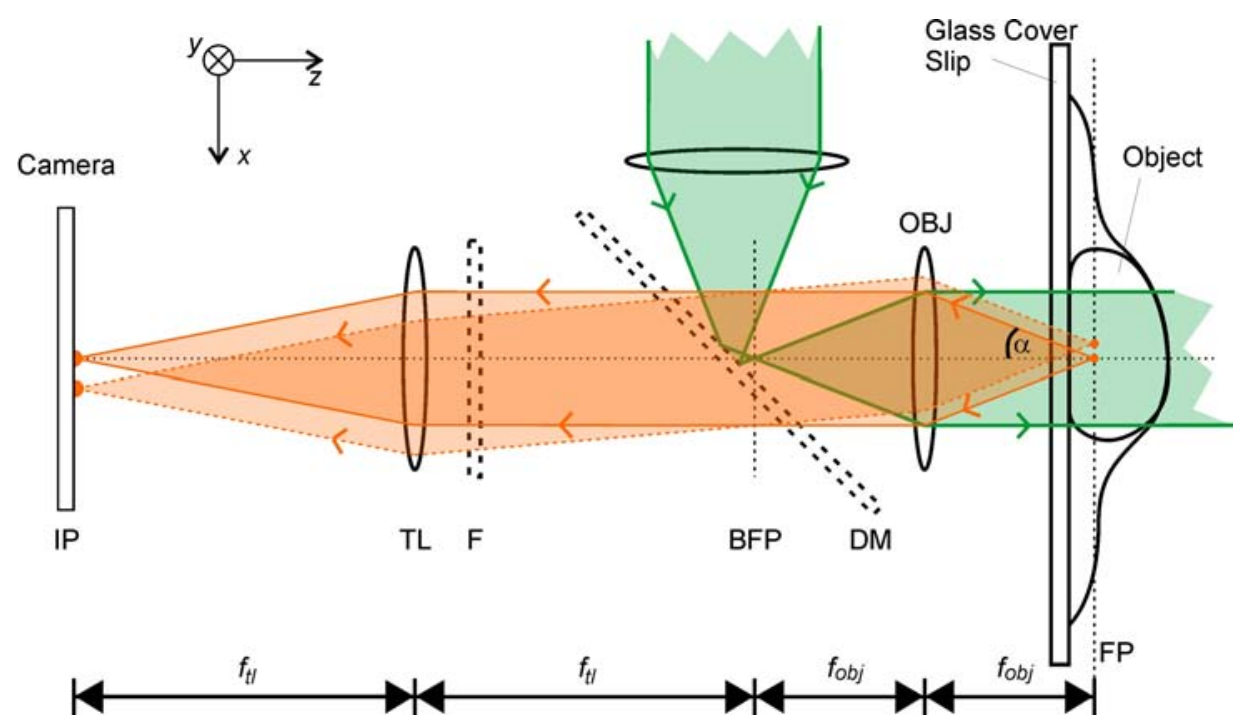

Figure 1.2: Typical wide-field microscope setup. The excitation light is drawn in green the red-shifted fluorescence light in orange. The object is a cell mounted on a glass cover slip. The object is typically illuminated through the cover slip. Oil as immersion medium is usually used between objective and cover slip (not shown). FP: focal plane of the objective lens, OBJ: objective lens with focal distance $f_{o b j}$, here simplified as a single lens, DM: dichroic long-pass mirror, BFP: back focal plane of the objective, F: fluorescence filters, TL: tube lens with focal distance $f_{t l}$, IP: image plane. $\alpha$ : Semiaperture angle of the objective lens. The central horizontal dotted line represents the optical axis. For further details see text.

rescence light coming from the object (orange dashed and full lines for two exemplary points of the object) is collected by the same objective and separated from scattered excitation light by a dichroic long-pass mirror (DM), which reflects the light below a certain threshold wavelength and transmits the light above, thereby exploiting the Stokes-shift of the fluorophore. The object is placed in the focal plane of the objective so that the fluorescence light from there leaves the objective collimated. Typical objectives are optimised for this configuration. A so called 'tube lens' (TL) is needed to form a real image of the fluorescent marker distribution of the object. It is placed at its focal distance $f_{t l}$ apart from the back focal plane of the objective lens. To record the image, a camera is placed directly in the image plane (IP) of TL. Alternatively, the image can be further projected from the image plane onto the retina of the experimentalist's eye by an ocular and the lens of the eye. Usually, additional filters (F) are introduced which transmit only the fluorescence signal and block residual excitation light and other background light. The magnification factor $m$ of the image is given by the ratio of the focal distances of the objective (which in this thesis is always $f_{o b j}=2 \mathrm{~mm}$ ) and the tube lens $f_{t l}$, thus $m=f_{t l} / f_{o b j}$. The magnification is chosen such that a reasonable sampling of the image is given by the pixels of a given detection camera. In the following theoretical considerations, a magnification of $m=1$ will be assumed for simplicity. 
(a)

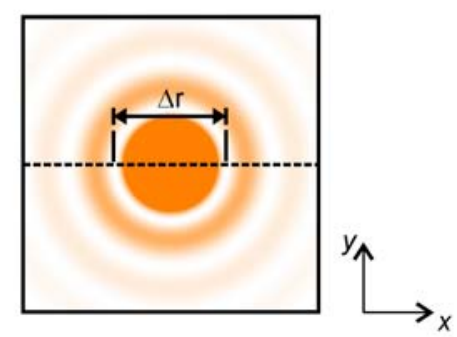

(c)

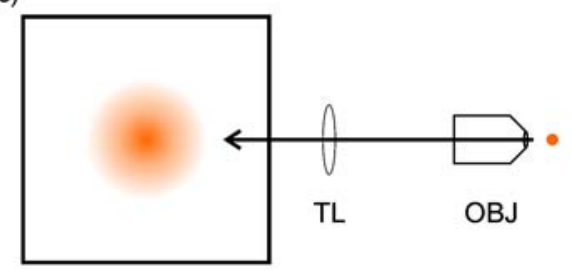

(b)

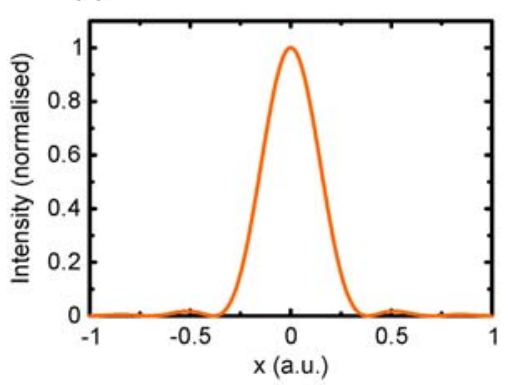

(d)

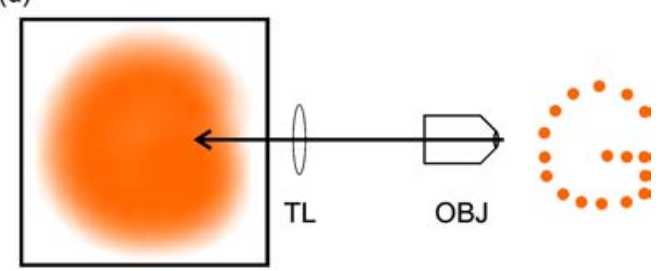

Figure 1.3: Point Spread Function and image formation of a wide-field microscope. (a) Shape of Airy pattern. Note: The central part is saturated to render the weak rings visible. (b) Line profile along the dotted line in (a). The Airy pattern consists of the major central peak and weak rings. (c) Image of a fluorescing pointobject. OBJ: objective lens, TL: tube lens. The point-object is not imaged as a point again but as an extended PSF-shaped spot. (d) Blurred, diffraction limited image of an object consisting of multiple point sources.

\subsubsection{Point Spread Function and Image Formation}

According to Abbe's rule shown in equation 0.1, the resolution of a microscope is limited. The reason is that any point-like object will not be imaged again as a point but as an extended circular spot. This spot is called the point spread function (PSF) of the detection beam path which is unique for each microscope and depends on its optical components. It is governed by the diffraction at the aperture of the objective $[32,33,34,35,36]$ and thus has the shape of the characteristic Airy-pattern known from diffraction at a circular aperture, which is shown in figure 1.3 (a) and in figure 1.3 (b) in a line profile. The shape of this detection PSF can be described by first order Bessel functions [32]. The distance between the first minima is given by

$$
\Delta r=1.22 \frac{\lambda}{\mathrm{NA}}
$$

where NA $=n \cdot \sin (\alpha)$ is the numerical aperture of the objective lens, $n$ denotes the refractive index of the surrounding medium and $\alpha$ is the semiaperture angle of the light entering the lens (shown in figure 1.2] [32]. The distance $\Delta r$ is marked in figure 1.3.a).

According to the so called Rayleigh-criterion, two spots can still be discerned if the maximum of one falls into the first minimum of the second one, that is if they are at least $\Delta r / 2$ apart, leading to the resolution of

$$
\Delta r=0.61 \frac{\lambda}{\mathrm{NA}}
$$


This equation is in accordance with Abbe's formula in equation 0.1 . The different factors 0.5 and 0.61 in the two equations only stem from the fact that Abbe assumed a square aperture as opposed to the circular aperture in this case.

The Airy pattern is often approximated by a Gaussian function, fitting the central part of the Airy disc quite well and omitting only the weak 'rings'. The resolution of the microscope is then given by the full width at half maximum (FWHM) of the Gaussian, which represents the minimum distance where two neighbouring Gaussians can still be separated.

An extended image $I(x, y)$ of an object consisting of a multitude of markers is given as the convolution of the marker distribution $O(x, y)$ of the object with the detection PSF, $h_{\text {det }}(x, y)$ :

$$
I(x, y)=h_{\text {det }}(x, y) \otimes O(x, y)
$$

Therefore, features smaller than the size of the PSF will be blurred and cannot be resolved. This is sketched in figure 1.3(c) and (d). Figure 1.3 (c) shows the image of a single point source and figure $1.3(\mathrm{~d})$ the resulting blurred image for a simple sample object with subdiffraction sized features. A sharp image would only be obtained, if $h_{d e t}(x, y)$ was the deltafunction. Since $\Delta r \rightarrow 0$ is not possible, this is not the case.

In the preceding consideration, only imaging of objects directly in the focal plane was treated. For objects out of the focal plane, the width of the PSF increases and their images will appear even more blurred. The thickness $\Delta z_{\text {dof }}$ of the layer from where the images are still acceptable is called 'depth of focus' or 'depth of field'. A typical definition of its thickness is [37]:

$$
\Delta z_{\text {dof }}=2 \frac{\lambda}{\mathrm{NA}^{2}}
$$

A detailed description is given for example by Meschede [37].

\subsection{Confocal Microscopy}

\subsubsection{Typical Microscope Setup and Imaging}

The wide-field microscopy concept as described above suffers from the fact that light from out-of-focus layers is not filtered out. Due to the homogeneous excitation of the whole sample laterally but also in all axial layers, the signal from the markers in the focal plane is thus overlayed by the signal from out-of-focus planes. Imaging thick samples, that is samples thicker than the depth of field, can therefore represent a problem.

The invention of confocal scanning microscopy reduced this problem [38]. Figure 1.4 shows a typical setup. The object is not illuminated homogeneously but the excitation light (green) is focussed to a small spot. For this, the light is sent collimated into the objective. The region of interest to be imaged is situated in the focal plane of the objective. The fluorescent light (orange lines) is collected by the objective lens and decoupled from the excitation light by a dichroic mirror. The light stemming from the focal plane of the objective (solid orange line) is collimated behind it and is focussed into the image plane by the tube lens. A detector such as an avalanche photo diode (APD) is placed on the beam axis behind a small pinhole to record the fluorescence light from the markers in the focal spot. The pinhole on the beam 


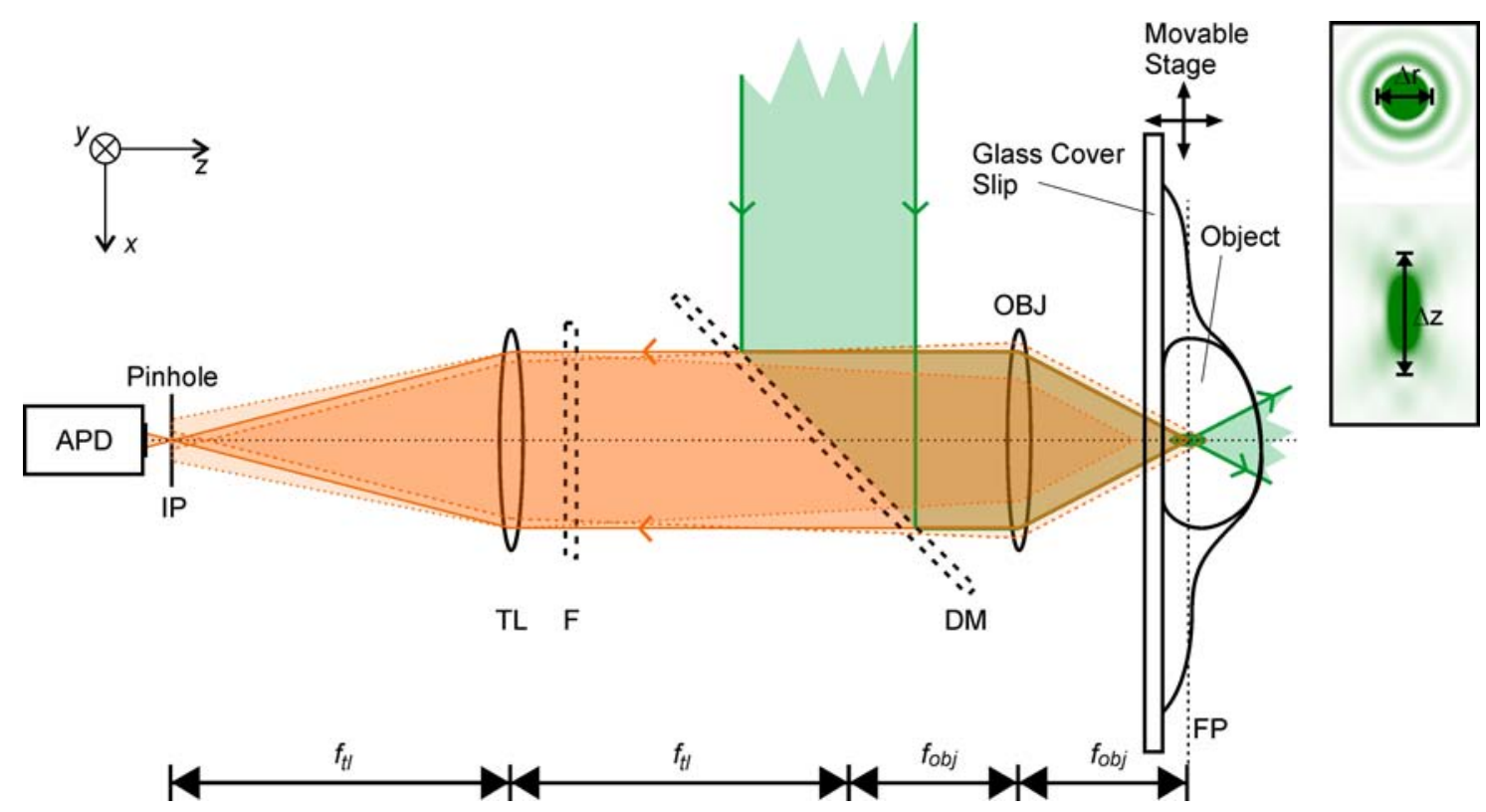

Figure 1.4: Typical confocal microscopy setup. The excitation light is drawn in green, the fluorescent light in orange. The object (a cell) is mounted on a glass cover slip which in turn is mounted on a movable stage. FP: focal plane of the objective lens, OBJ: objective lens with focal distance $f_{o b j}$, here simplified as a single lens, DM: dichroic long-pass mirror, F: fluorescence filters, TL: tube lens with focal distance $f_{t l}$, IP: image plane, APD: avalanche photo diode as detector. The box on the right side shows the shape of the excitation PSF in the lateral direction ( $x y$-plane) on top and in the axial direction on the bottom. Note that the latter plot is rotated by $90^{\circ}$ with respect to the main image. $\Delta r$ and $\Delta z$ are the distances between the first two minima. Note that the intensity distribution in the central parts of both PSFs is saturated in order to also visualise the weak sidelobes. For further details see text.

axis is located exactly in the focal plane of the tube lens and has approximately the size of the detection PSF in that plane. Thus only light stemming from the focal layer of the objective lens fully passes the pinhole. Light from out of-plane-layers (dashed and dotted orange lines) is not focussed in the plane of the pinhole and thus effectively filtered out.

In order to record the whole region of interest, the spot can be scanned over it by changing the angle of the incident beam in the back focal plane of the objective, for example by means of scanning mirrors. More simply, the object can be moved step by step through the spot using a piezo-actuated stage on which the object is mounted. For each scanned pixel, whose location is known, the recorded intensity is saved by a computer and the image can be reconstructed according to this data, usually showing the recorded intensity per pixel in a colour coding to illustrate the density distribution of the structures.

\subsubsection{Resolution of a Confocal Microscope}

One can regard the focussed excitation spot as a probe, which scans the sample non-invasively in all spatial directions. In analogy to the PSF of the detection beam path discussed in section 1.2.2, also this focussed spot, the excitation PSF, is subject to the diffraction limit. Thus its 
lateral size is also given by

$$
\Delta r=1.22 \frac{\lambda}{n \sin \alpha}
$$

The axial extent amounts to [35]

$$
\Delta z=4.00 \frac{\lambda}{n \sin ^{2} \alpha} .
$$

Both values are defined as the distances between the first two minima in the corresponding direction, as shown in the projections of the PSF in the box on the right side of figure 1.4 . Effectively, the resolution of a confocal microscope is determined by the combination of the normalised excitation PSF $h_{e x c}$, the normalised detection PSF $h_{d e t}$ and the detection pinhole of diameter $p$. The resulting confocal PSF $h_{\text {conf }}$ is thus:

$$
h_{\text {conf }}=h_{e x c} \cdot\left(h_{\text {det }} \otimes p\right)
$$

The image $I(x, y)$ of an extended object in the $x y$-plane with the marker distribution $O$ is then

$$
I=h_{\text {conf }} \otimes O=\left(h_{\text {exc }} \cdot\left(h_{\text {det }} \otimes p\right)\right) \otimes O .
$$

Since the pinhole is in practice of finite size roughly on the order of the size of the Airy disk of the detection PSF in the image plane, $h_{\text {det }} \otimes p$ is a rather wide distribution so that one can approximate

$$
h_{\text {conf }}(x, y) \approx h_{\text {exc }}(x, y)
$$

in lateral direction. In axial direction however, the pinhole serves as a very efficient filter for light from out-of-plane regions. Here, $h_{\text {conf }}(z) \approx h_{\text {exc }}(z) \cdot h_{\text {det }}(z)$. Due to the almost Gaussian shape of $h_{\text {exc }}(z)$ and $h_{\text {det }}(z)$, the axial extent of $h_{\text {conf }}$ is thus smaller than the one of $h_{\text {exc }}$, leading to a resolution of $\approx 500 \mathrm{~nm}$ in axial direction for visible light. Equation 1.8 shows that also in the confocal case any structures smaller than the diffraction limit will appear to be blurred in the image since the relatively large probing excitation spot averages the signal from all points within.

Although the axial resolution is still worse than the lateral resolution, confocal scanning microscopy represents an improvement over the wide-field-configuration, which shows no signal filtering for fluorescence light from out-of-focus regions.

\subsection{Breaking the Diffraction Barrier with RESOLFT-Microscopy}

\subsubsection{The General Principle}

Typical RESOLFT-microscopes follow the same imaging principle as confocal scanning microscopes. Although the RESOLFT-concept is more general and camera-based wide-field configurations are also possible [39, 40], in most experimental realisations the image is 
formed by scanning one or multiple focussed light spots over a sample step by step in a defined way and recording the signal at each point [12, 13, 41]. The central idea of RESOLFT, however, is to use a switching process between two discernable states of a given marker in order to reduce the effective extent of the probing focal spot to subdiffraction size. This way, a resolution beyond the diffraction barrier is achieved. The confocal pinhole is not necessarily needed for the superresolution concept but it does in practice reduce background light from out-of-focus areas and is therefore typically implemented in actual experimental setups.

The RESOLFT-concept is not limited to fluorescent markers. Scattering processes for example might also be exploited [11]. Up to now, however, only fluorescent markers were used due to their excellent signal to noise ratio. The following introduction to RESOLFTmicroscopy will therefore refer to this specific case for simplicity. The concept is strikingly simple and leads to the breaking of Abbe's diffraction barrier by purely physical means, without the need for computational image reconstruction.

Let us assume that it is possible to switch the fluorescent marker between a fluorescent 'on'state and a discernable 'off'-state. The latter state can be a completely non-fluorescent state or just a state with a different fluorescence spectrum, which can be filtered from the signal in the 'on'-state. One can now reduce the size of the diffraction limited excitation spot by switching all markers in the excitation spot to the off-state except for those in its central region. This leads to an effectively much smaller probing spot which thus can resolve smaller details of the sample. This switching process is performed with light in order to preserve the non-invasiveness of the imaging system on the one hand and to be able to control the switching process spatially on the other by applying appropriate light patterns. The switch-off light is typically applied in such a way that it has zero intensity in the centre of the excitation spot, but as high intensity as possible in the other regions of the excitation spot to ensure effective switching. These two requirements, that is switching of the marker between a bright and a dark state using light and a way to create a switch-off light distribution with a perfect zero intensity node are the key features of a RESOLFT-microscope. In the following, first a method of how to create this light distribution will be introduced and then in section 1.4 .3 the general mechanism and kinetics of the switching process will be explained.

\subsubsection{Creation of Switch-off PSF by Phase Modulation}

The switch-off PSF with the mandatory zero intensity node can be created by proper manipulation of the phase of the switch-off light before it falls onto the objective lens [42, 43].

If the incident light has a circular polarisation and a perfect planar wavefront, the light will be focussed to a spot circular in the $x y$-plane and elliptical in the perpendicular planes, yielding the standard excitation PSF. The projections in the respective planes are shown in figure $1.5(a)$.

A central zero intensity node can be created if the phase of one half of the beam is retarded by the factor of $\pi$ relatively to the other. In that case, each part of the beam experiences destructive interference with its counterpart from the opposite side of the beam in the centre of the focal spot. This is shown in figure 1.5 (b) for light linearly polarised in the $y$-direction. 


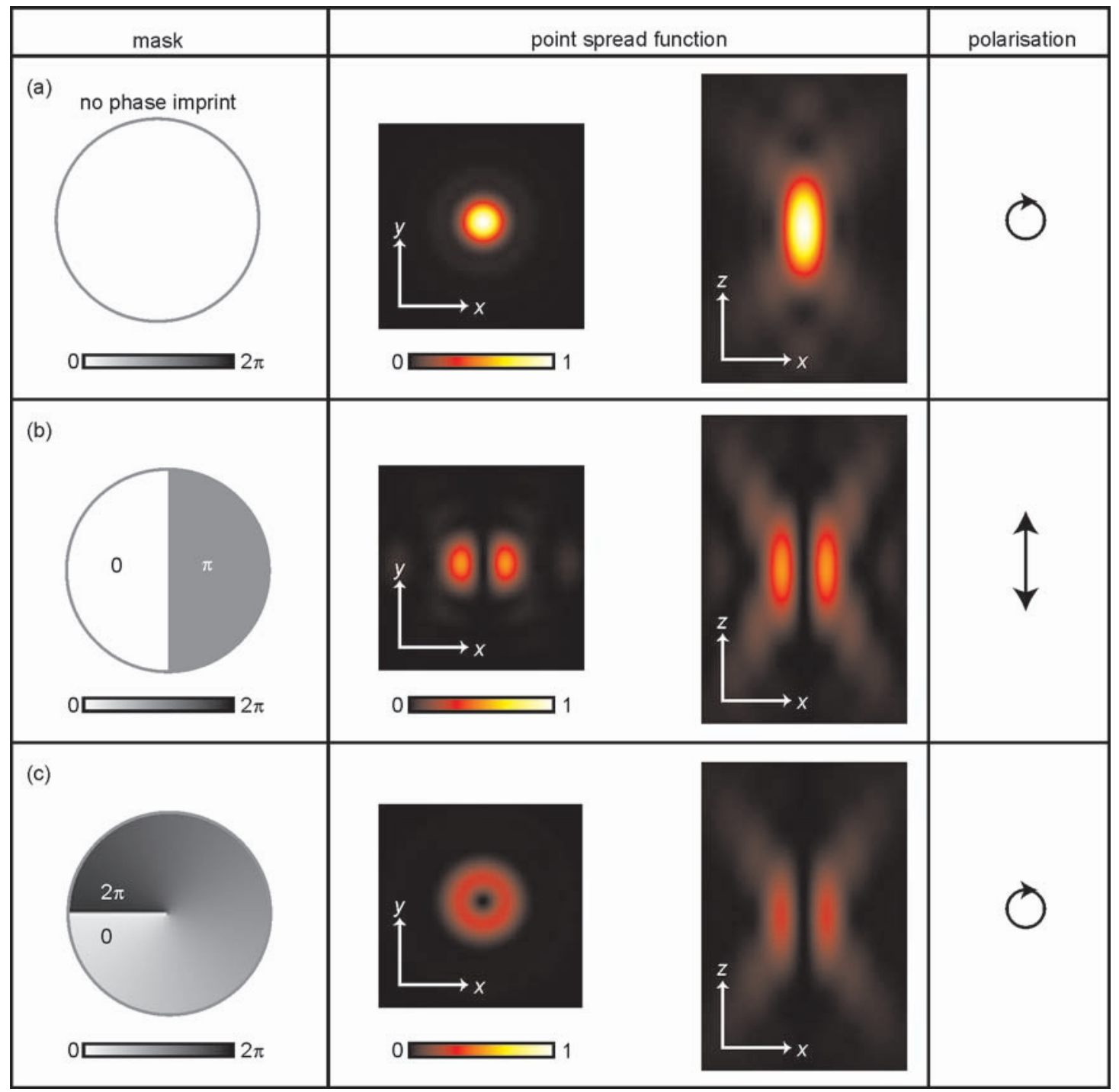

Figure 1.5: Effect of phase manipulation on the focal spot. The intensity distribution is drawn in colour coding from black to white via red and yellow for increasing intensity. Panel (a) shows the unaltered focal spot in the lateral $x y$-plane and in the perpendicular $x z$-plane for circularly polarised light. Panel (b) shows the focal spot for linearly polarised light if the phase of one half of the incident beam on the objective lens is retarded by $\pi$ by means of a phase manipulation plate shown on the left. The PSF features a central line with zero intensity in $y$-direction. Panel (c) displays the effect of a helical phase retardation from 0 to $2 \pi$ on circularly polarised light in the focal spot. The lateral projection of this PSF clearly shows the central zero intensity node. Image adapted from [44] with friendly permission of B. Harke. 
The left panel shows the applied phase imprint. Destructive interference leads to a line in the $y$-direction with zero intensity as shown in the middle panel. This resulting switch-off PSF can be used for one-dimensional resolution enhancement.

A laterally radially symmetric switch-off PSF applicable for two-dimensional resolution enhancement can be gained by using circularly polarised light and applying a helical phase imprint from 0 to $2 \pi$ onto the wave front as shown in 1.5 (c). The resulting focal spot features a central zero. Again, those parts of the incident beam which are opposite to each other with the same distance from the centre always have a relative phase of $\pi$ and thus experience destructive interference. In the lateral projection, the resulting intensity distribution has a characteristic toroidal shape and is therefore often referred to as 'doughnut'-PSF. When centrally aligned with the excitation PSF, this switch-off light distribution thus leads to the desired lateral constriction of the excitation spot.

The required phase retardation patterns can be created simply by coating a glass substrate with a substance whose diffraction index $n>1$, for example with a polymer or $\mathrm{MgF}_{2}$. The thickness of the coating then defines the phase shift. A more general way is to use a spatial light modulator (SLM), which is a programmable device based on liquid crystals which can produce arbitrary phase patterns. An advantage is here that not only the phase pattern can be imprinted but also undesired disturbances of the wavefront can be corrected at the same time. Whereas the first method works in 'transmission mode', that is the light passes through the substrate, the SLM that was used in the later experiments works in 'reflection mode', that is the light is reflected by a built-in mirror and passes the phase retarding liquid crystal layer twice.

\subsubsection{The Switch-off Kinetics and Resolution of a RESOLFT-Microscope}

In the following, the kinetics of the switch-off process will be elucidated and the effective PSF of the RESOLFT-Microscope will be calculated [39, 45, 46, 47, 48]. Let us imagine the simple case of a fluorescent molecule which has a fluorescent on-state $A$ and a nonfluorescent off-state $B$. The transition $A \rightarrow B$ shall be driven by light with the rate $k_{A B}=$ $\sigma_{A B} I_{A B}$, where $\sigma_{A B}$ is the cross-section for the process and $I_{A B}$ the intensity of the light which is used to induce this 'off'-transition [45]. The rate for the reverse transition is $k_{B A}$. It may also be driven by light but can as well be induced by chemical, thermal or any other suitable processes. The kinetics of the molecular states A and B are then given by

$$
d N_{A} / d t=-d N_{B} / d t=k_{B A} N_{B}-k_{A B} N_{A},
$$

where $N_{A, B}$ are the normalised population probabilities for the two states, that is $N_{A}+N_{B}=$ 1. With the initial condition that $N_{A}(t=0)=1$, where $t$ denotes the time, the solution of this differential equation is

$$
N_{A}(t)=\frac{k_{B A}}{k_{B A}+k_{A B}}+\frac{k_{A B}}{k_{B A}+k_{A B}} \exp \left[-\left(k_{B A}+k_{A B}\right) \cdot t\right] .
$$

Since the reverse reaction may also be driven by light, there is in practice usually a certain cross-talk from the switch-off light to also induce the 'on'-transition. We therefore get $k_{B A}=$ 
$\tilde{k}_{B A}+k_{B A}^{\lambda}=\tilde{k}_{B A}+\sigma_{B A} \cdot I_{A B}$, where $\tilde{k}_{B A}$ is the rate associated to all non-light-driven processes and $k_{B A}^{\lambda}=\sigma_{B A} \cdot I_{A B}$ the rate for the light driven on-transition, with $\sigma_{B A}$ being the cross-section for this undesired cross-talk. The general form of $N_{A}$ is thus:

$$
N_{A}=\frac{\tilde{k}_{B A}+k_{B A}^{\lambda}}{\tilde{k}_{B A}+k_{B A}^{\lambda}+k_{A B}}+\frac{k_{A B}}{\tilde{k}_{B A}+k_{B A}^{\lambda}+k_{A B}} \exp \left[-\left(\tilde{k}_{B A}+k_{B A}^{\lambda}+k_{A B}\right) \cdot t\right] .
$$

For different molecular switching mechanisms, different approximations can be made to simplify this equation. Here, only the case relevant for the later RESOLFT experiments with photoswitchable organic dyes will be treated, where the two states A and B are long-lived. Thus $\tilde{k}_{B A} \ll k_{B A}^{\lambda}, k_{A B}$. For sufficiently high $I_{A B}, \tilde{k}_{B A}$ may therefore be neglected. We obtain the solution

$$
N_{A}=\frac{\sigma_{B A}}{\sigma_{B A}+\sigma_{A B}}+\frac{\sigma_{A B}}{\sigma_{B A}+\sigma_{A B}} \exp \left[-\left(\sigma_{B A}+\sigma_{A B}\right) I_{A B} \cdot t\right] .
$$

One can read off equation 1.13 that for an applied light energy $I_{A B} \cdot t \gg\left(\sigma_{B A}+\sigma_{A B}\right)^{-1}$ the system reaches a purely photostationary state $N_{A}^{\infty}=\sigma_{B A} \cdot\left(\sigma_{B A}+\sigma_{A B}\right)^{-1}$. The absolute switch-off efficiency is thus only dependent on the ratio of $\sigma_{B A}$ and $\sigma_{A B}$. These values are usually dependent on the wavelength of the applied light. In the ideal case this ratio can be tuned to $\sigma_{B A} \ll \sigma_{A B}$ by choosing an appropriate wavelength, so that the residual fluorescence of the photostationary state is negligible in the experiment. We then get the simple dependency

$$
N_{A}=\exp \left[-\sigma_{A B} \cdot I_{A B} \cdot t\right],
$$

showing that virtually all molecules can be driven to their dark state by light. In a typical RESOLFT-imaging experiment as performed in this thesis, the illumination time $t$ for each pixel is fixed and we can therefore assume it to be a constant $t_{0}$. The residual fraction of markers in the 'on'-state then just depends on the applied light intensity $I_{A B}$.

It is useful to define a characteristic switch-off light intensity $I_{\text {sat }}$, where $N_{A}\left(I_{\text {sat }}\right)=1 / 2$. Equation 1.14 then leads to

$$
I_{\text {sat }}=\frac{\ln (2)}{t_{0} \cdot \sigma_{A B}} .
$$

Figure 1.6(a) shows the $x y$-intensity distribution of the excitation PSF on the left side. The right side displays the standard doughnut-shaped switch-off PSF. In practice, both beams are centrally aligned. Figure 1.6(b) displays a line profile of the aligned beams with full lines, green corresponds to the excitation beam, black to the switch-off beam. In the RESOLFTexperiments described in this thesis, the excitation of the residual markers in the on-state is performed after the switch-off process. The lateral effective PSF $h_{e f f}$ of the RESOLFT microscope can then be calculated as follows, where we can reduce the lateral spatial variables to one, due to the rotational symmetry of the two PSFs:

$$
h_{e f f}(x, I)=h_{e x c}(x) \cdot \eta\left(h_{o f f}(x) \cdot I\right)
$$

Here, $h_{e x c}(x)$ denotes the normalised excitation PSF, $h_{o f f}(x)=I_{A B}(x) / I$ the normalised switch-off PSF with $I$ being the maximum of the intensity distribution and $\eta=\eta\left(I_{A B}(x)\right)=$ 

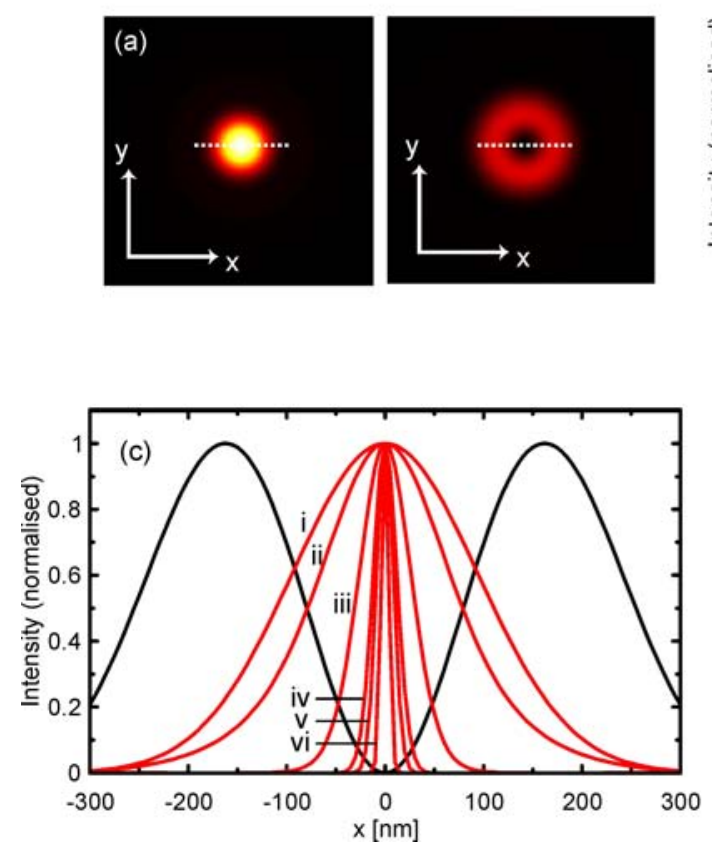
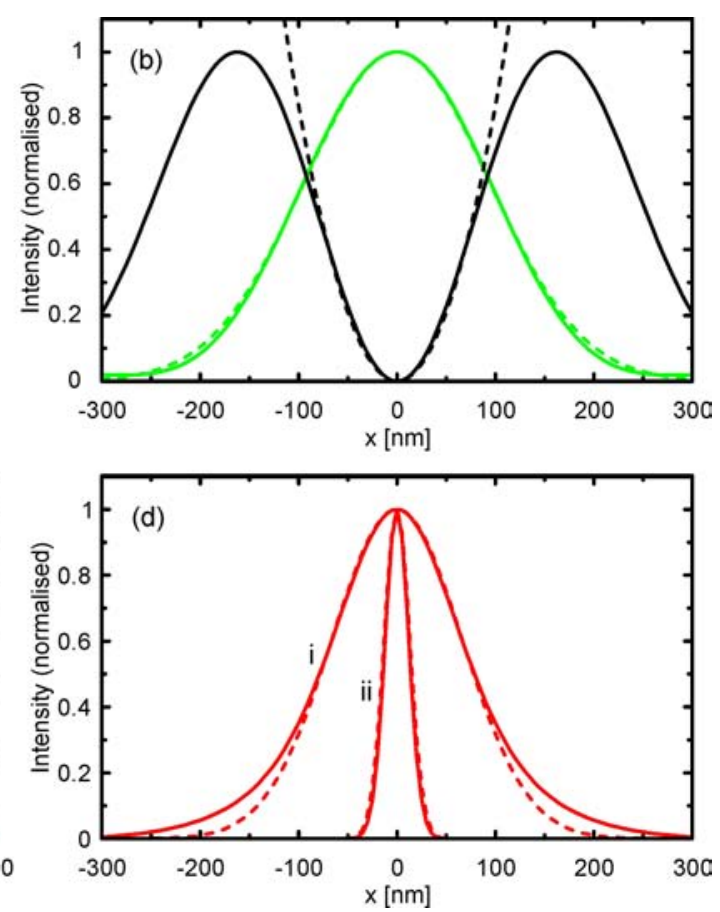

Figure 1.6: Resolution enhancement by switching off the markers which are not in the centre of the excitation spot. (a) Lateral projection of the excitation PSF (left) and the lateral projection of a switch-off PSF featuring the desired nodal intensity zero (right). Both PSFs are calculated. (b) Intensity profile along the $x$-axis as shown with the dotted lines in (a). The excitation spot is shown with a green solid line and its Gaussian approximation with a dashed green line. The switch-off beam profile $h_{o f f}(x)$ is drawn in black, the solid line showing the actual PSF and the dashed one the paraboloid approximation of its centre. (c) Effective PSF $h_{\text {eff }}$ for $\zeta=0,1$, $10,50,100,500$ with the respective red plots (i), (ii), (iii), (iv), (v), (vi). $h_{o f f}$ is again plotted with a black line. (d) Illustration of the validity of the paraboloid approximation of $h_{\text {off }}(x)$ especially for higher values of $\zeta$. (i) and (ii) represent $h_{\text {eff }}(x)$ for $\zeta=1$ and $\zeta=50$, respectively, for the actual switch-off PSF in a solid line and the paraboloid approximation in a dashed line.

$\eta\left(h_{o f f}(x) \cdot I\right)$ the switch-off function which corresponds to the relative number of molecules still in the fluorescent state $A$, here

$$
\eta\left(h_{o f f}(x) \cdot I\right)=N_{A}\left(h_{o f f}(x) \cdot I \cdot t_{0}\right) .
$$

The resolution $d$ of the RESOLFT-microscope can be expressed as the FWHM of $h_{\text {eff }}(x)$. To derive a simple expression for $d$, we can very well approximate the excitation PSF $h_{\text {exc }}$ by a Gaussian with the same FWHM $d_{c}$ as the excitation PSF:

$$
h_{e x c}(x)=\exp \left[-\frac{4 \ln (2) x^{2}}{d_{c}^{2}}\right]
$$

According to equation 1.9, the confocal PSF can be approximated by the excitation PSF in lateral directions. Therefore $d_{c}$ denotes the resolution of a standard confocal microscope. The function $h_{\text {exc }}(x)$ of equation 1.18 is shown in figure 1.6 (b) with the dashed green line. 
The switch-off PSF can be approximated by a parabola in the relevant region close to $x=0$, that is $h_{o f f}(x)=4 a^{2} x^{2}$. Figure 1.6(b) shows this approximation as a dashed black line. Inserting these two functions into equation 1.16 and using equations 1.14 and 1.15 yields:

$$
\begin{aligned}
h_{e f f}(x, I) & =\exp \left[-\frac{4 \ln (2) x^{2}}{d_{c}^{2}}\right] \cdot \exp \left[-4 a^{2} \sigma_{A B} I t_{0} x^{2}\right] \\
& =\exp \left[-4 \ln (2)\left(\frac{1}{d_{c}^{2}}+a^{2} \frac{I}{I_{\text {sat }}}\right) x^{2}\right]
\end{aligned}
$$

This Gaussian approximates the effective PSF of a RESOLFT-type high resolution microscope. The FWHM $d$ of the Gaussian in equation 1.19 is

$$
d=\left(\frac{1}{d_{c}^{2}}+a^{2} \frac{I}{I_{s a t}}\right)^{-1 / 2}=\frac{d_{c}}{\sqrt{1+d_{c}^{2} \cdot a^{2} \cdot \zeta}},
$$

where the saturation factor $\zeta=I / I_{\text {sat }}$ has been introduced [48]. $d$ can be directly seen as the resolution of the microscope. The resolution is not limited by any physical principles, it just depends on the value of $\zeta$ and thus on $I$.

Figure 1.6(c) shows the effective PSF according to this model for several values of $\zeta$. These approximations are particularly good for high values of $\zeta$ as shown in figure 1.6(d). The figure shows the effective PSF $h_{e f f}(x)$ for values of $\zeta=1$ (i) and $\zeta=50$ (ii) in solid black lines. The dashed lines correspond to the effective PSF calculated using the paraboloid approximation of $h_{o f f}$.

For imaging in practice, the constriction process has to be applied for every pixel and therefore needs to be reversible. In the case of the photoswitchable dyes used in this work, the reactivation is also induced by light but of a different wavelength than the excitation and switch-off light.

We can specify the two basic requirements for RESOLFT-microscopy as:

- A marker dye which is reversibly photoswitchable between a detectable on-state and a non-detectable off-state with a high signal contrast between the two.

- A light distribution featuring an intensity zero which induces the switch-off process.

\subsection{Breaking the Diffraction Barrier with SMS-Microscopy}

\subsubsection{The General Principle}

Analogous to RESOLFT, the key to resolution enhancement in SMS-type microscopy is the exploitation of the switchability of appropriate markers between two distinguishable states, which will also be called on- and off-state, respectively [11]. So far, in the experimental realisations, the bright and dark states of photoswitchable fluorescent markers were used as the on- and off-states, respectively. Therefore, fluorescent markers will be assumed in the following. However, in analogy to RESOLFT-microscopy, the SMS-concept is in principle 

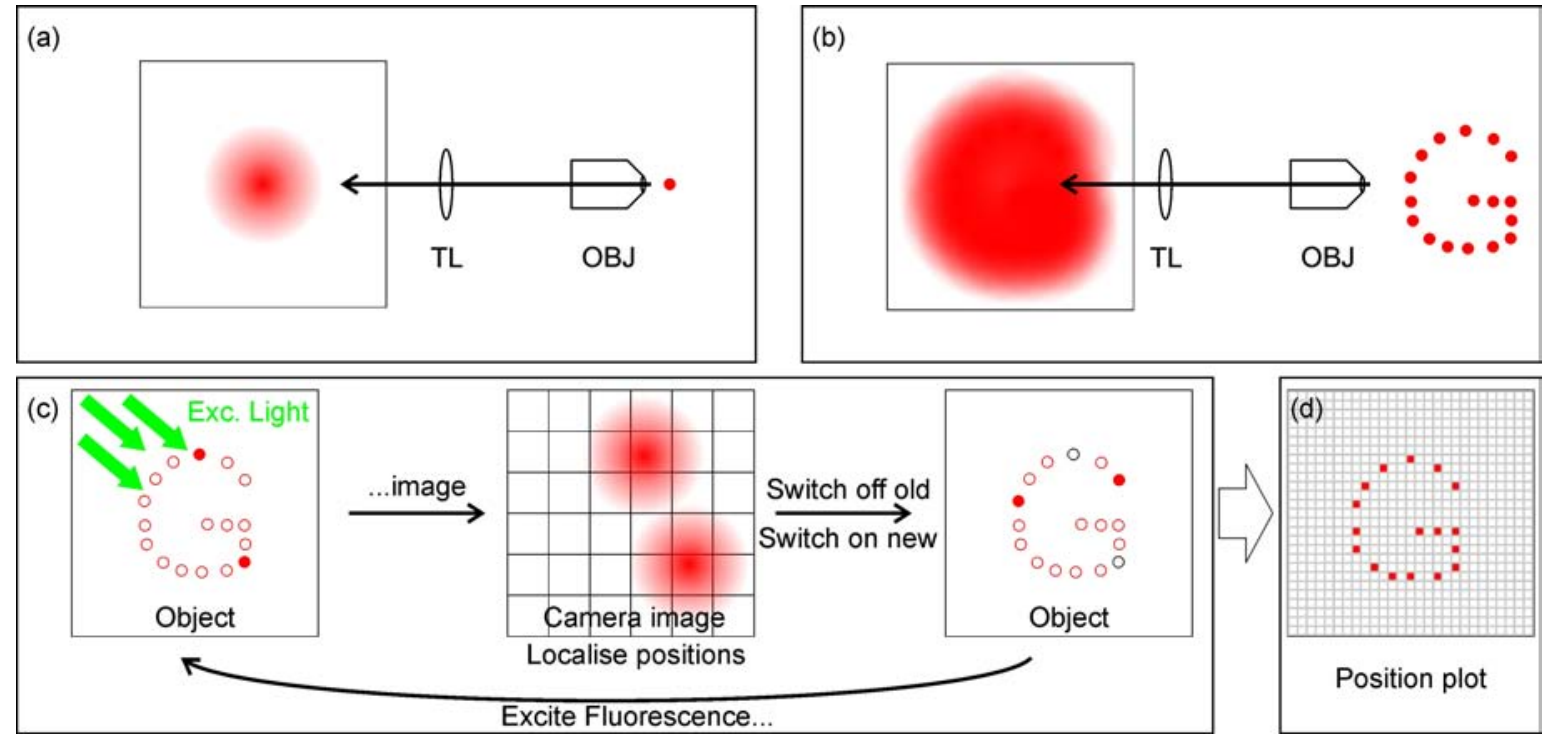

Figure 1.7: (a) Image of a point-like object, such as a single fluorescent dye, on a screen. (b) Complex objects comprising multiple markers with features smaller than the PSF appear blurred and their features cannot be resolved. (c) Left: Sparse subset of activated dyes (filled circles) which fluoresces when excited by light as opposed to the majority of deactivated dyes (hollow circles). Middle: The activated markers are distributed so sparsely that their image PSFs are spatially separated. The grid denotes the camera pixel. An image is recorded and the position of the markers is determined and saved. Right: After imaging, the dyes have to be switched off (black hollow circles) and a new sparse subset is activated, which again can be imaged. This process is repeated until the image is complete. (d) Illustration of the image reconstruction by plotting the registered dye positions in a two-dimensional histogram. The grey grid serves to highlight the histogram bins.

also not limited to fluorescent markers [11].

Typical SMS-microscopes are based on standard wide-field microscopy. It was discussed in section 1.2.2 how the image is formed as a convolution of the PSF and the marker distribution and how subdiffraction sized features are thus blurred. This effect is again illustrated in figure 1.7(a) and (b). SMS-microscopy circumvents this problem by using the switchability of the markers to isolate adjacent ones and image them individually [14, 15, 16]. This process is displayed in figure 1.7(c). Let us assume that initially all markers but a small subfraction can be driven into their off-state (open circles). The markers in the on-state (filled circles) are randomly distributed over the sample. This subfraction of markers has to be so small that the probability of two markers in the on-state being closer than the size of the PSF is negligible. We can now excite the markers in the on-state and record an image of them, yielding the corresponding distribution of clearly separated diffraction limited spots. Given a good signal, that is a sufficient number of photons emitted by the marker, the centre position of these spots can be determined with an accuracy corresponding to only fractions of the actual size of the PSF [49, 50, 51]. Using the information that each of these spots is generated by just a single marker, their position is thus obtained with sub-diffraction localisation precision. After switching off these markers (black open circles), a new sparse subset can be activated, imaged and localised. This process can be repeated multiple times until sufficient spatial information about the object is collected. The resulting high-resolution image is obtained 


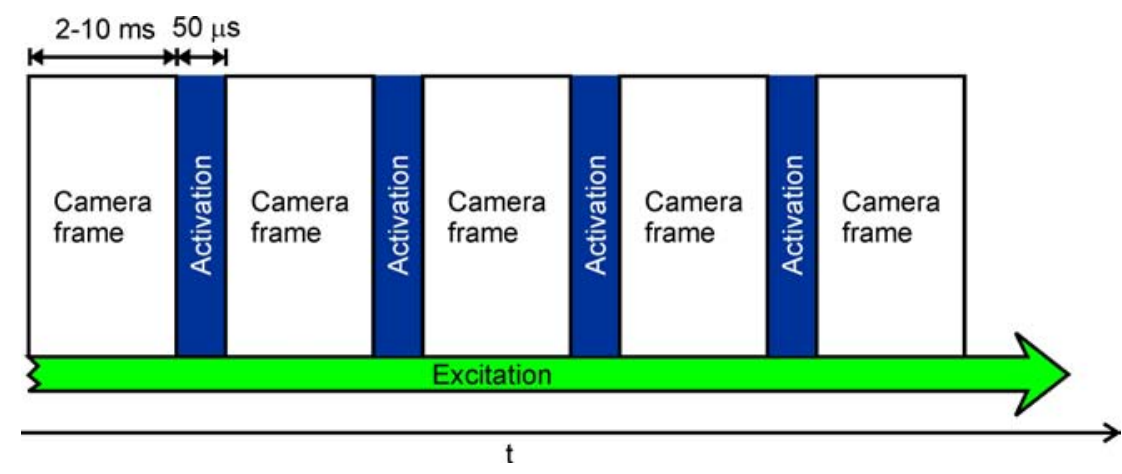

Figure 1.8: IRA imaging scheme as applied in this thesis. Time $t$ is plotted on the abscissa.

simply by plotting the positions of all the markers such as in 1.7 (d).

Activation and deactivation do not necessarily have to be controlled by light. Since spatial control of the switching process is not mandatory with this method, it may also be induced by other means such as temperature changes or changes in the chemical environment, as long as an adequate density of markers is in the on-state.

\subsubsection{Typical Image Acquisition}

The actual image acquisition used in this work is non-triggered, which means that it does not use fixed 'switch-on image switch-off'-cycles as indicated above. It is instead adapted to the peculiarities of the markers used. These cannot be switched off in an independent, controlled way. When excited after activation, which is either spontaneous or induced by light, they emit a burst of $\bar{N}$ photons on average before they are photobleached or go back to their long lived dark state.

With the applied image acquisition scheme, the object is continuously illuminated by the excitation light while the camera is running independently. Excitation intensity and the frametime of the camera $t_{\text {frame }}$ are optimised to the 'natural' on-off-cycles of the dyes used, so that the highest possible number of photons $N$ per molecular photon burst is recorded on the same frame but the total frame time remains as low as possible in order to achieve a low total image acquisition time. This scheme is displayed in figure 1.8 and is termed 'Independent Running Acquisition' (IRA) [17, 18]. In case activation of the dyes is needed, the light is favourably sent in during the camera dark time between the frames to reduce background induced by the activation light and to assure that the molecules are activated in the beginning of each frame. The frame transfer EMCCD camera used (IXON-Plus DU-860, Andor Technology) has a dark time of only $50 \mu$ s between the frames at typical $t_{\text {frame }}$ of $2-10 \mathrm{~ms}$. A typical camera frame is shown in figure 1.9. Unlike in the simple sketch shown in figure 1.7, a colour coding from black to white via red and yellow is used to visualise the signal intensity, that is the number of photons registered by each camera pixel, in finer nuances.

The varying intensities of the single molecule images is due to the fact that the photon distribution per burst follows a geometrical distribution, as will be discussed later on. 


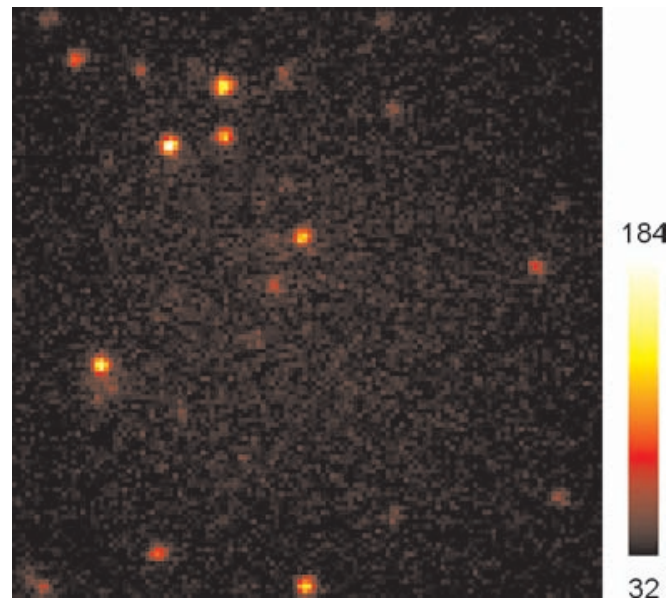

Figure 1.9: Typical single camera frame (raw data) of $128 \times 128$ pixels showing the signal of several single molecules (marker type SRA577, see section 3.1.1) in colour-coding. The colour bar denotes the number of photons per pixel. The frame is taken from the measurement for figure 3.5 (c).

\subsubsection{Localisation and its Precision}

The $N$ detected photons of a single molecule burst, also called 'event' within this thesis, are stochastically distributed on the detector in the image plane according to the probability distribution given by the normalised detection PSF of the microscope $h_{\text {det }}(x, y)$. In the example frame of figure 1.9 the single molecule PSFs can be easily made out. Even by eye, the localisation precision the centre of the spots is better than the size of the spots. In the following, the theoretical localisation precision and the resulting resolution in the final image as well as the computational localisation algorithm will be discussed.

Due to the rotational symmetry of the PSF, one spatial coordinate, which we call $r$, is sufficient for the following considerations on the localisation precision.

Each collected photon gives a measure for the position $r_{0}$ of a given marker with an error given by the standard deviation $s_{\text {det }}$ of the detection PSF $h_{\text {det }}(r)$, whose Gaussian approximation is

$$
h_{\text {det }}(r)=A \cdot \exp \left[-\frac{\left(r-r_{0}\right)^{2}}{2 \cdot s_{\text {det }}^{2}}\right],
$$

with $A$ denoting the amplitude. The actual centre of the measured PSF of an event can be estimated by the average of the positions of all the recorded photons with an error of [49, 50, 51]:

$$
\Delta r^{\prime}=\frac{s_{d e t}}{\sqrt{N}}
$$

To obtain a formulation for the actual resolution of an SMS-microscope it is necessary to consider the meaning of this localisation precision in the final image. We assume that we image and localise the same marker at position $r_{0}$ multiple times with $N$ photons for each event. If the localised positions are now plotted in the final image, their distribution will approximately form a Gaussian around $r_{0}$ with the standard deviation $\Delta r^{\prime}$ of equation 1.22 [52]. 


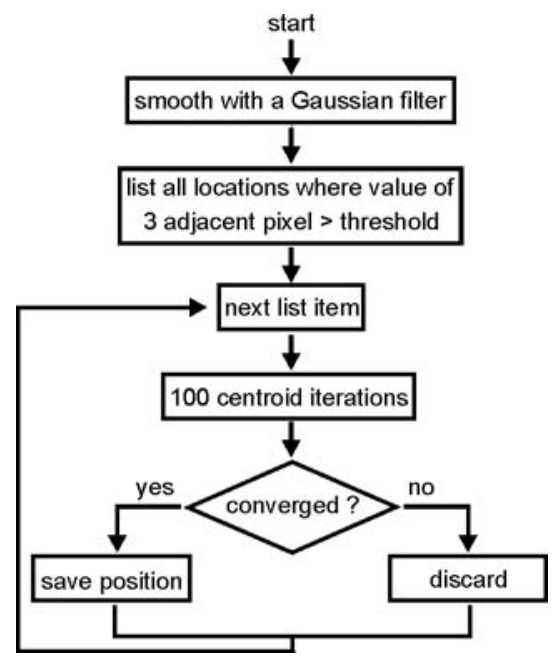

Figure 1.10: Flow diagram of the localisation analysis.

The standard deviation $\Delta r^{\prime}$ is related to the FWHM $\Delta r$ of this image Gaussian as $\Delta r=$ $2 \sqrt{2 \ln (2)} \cdot \Delta r^{\prime}$. Two adjacent markers can still be discerned in the image, if they are separated at least by a distance equal to $\Delta r$. Therefore, the relation

$$
\Delta r=\frac{d_{d e t}}{\sqrt{N}}
$$

gives a measure for the theoretically achievable resolution of an SMS-microscope, where $d_{d e t}=2 \sqrt{2 \ln (2)} \cdot s_{\text {det }}$ is the FWHM of the Gaussian approximation of the detection PSF. This estimation neglects all noise from background, dark counts, camera readout or any other sources. The camera readout noise can be reduced by applying an electron-multiplying CCD camera (EMCCD), which multiplies the signal before readout, thus enhancing the signal to readout noise ratio. However, due to this gain process, an additional excess noise factor of $\approx 2$ is introduced, leading to a theoretical resolution of

$$
\Delta \tilde{r} \approx \frac{d_{\text {det }}}{\sqrt{N / 2}}
$$

for the camera and the high gain values used in the experiments [53].

A scheme of the actual position determination algorithm is given in figure 1.10. First, the background of each camera frame is eliminated by substracting the same image frame after applying a Gaussian filter removing the high frequencies. In order to distinguish single molecule events from noise, all regions are then identified where more than typically three adjacent pixels are above a threshold of typically 10 photons. Each of these incidents is considered to be one single molecule event. A square with a side length of $\approx 5.5 \cdot d_{\text {det }}$ around each of the events is cut out and the Gaussian mask fit is applied on each of them [18, 50]. The starting point $\mathbf{r}_{0}$ of the fitting iteration is set to the highest pixel value. In each iteration, the centre of mass position $\mathbf{r}_{n}$ is determined after multiplying the data with a Gaussian with 
(a)

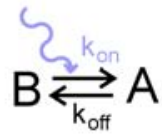

(b)

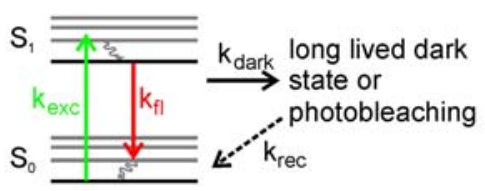

(c)

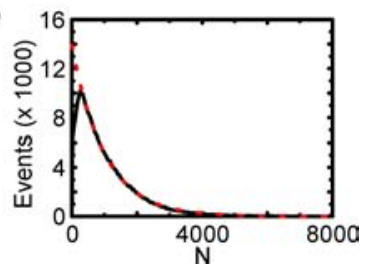

Figure 1.11: (a) Switching mechanism of typical photoswitchable SMS-dye as used in this thesis. A denotes a fluorescent on-state and B a non-fluorescent off-state. Initially, all dyes are assumed to be in state B. Transition $\mathrm{B} \rightarrow \mathrm{A}$ can be induced by light, the reverse transition is assumed to be of spontaneous nature. (b) Jablonski diagram of a dye in A. Switching it off again is practically done by photobleaching it or transferring the dye into a long lived dark state. (c) Geometric distribution of photon numbers $N$ per molecular burst shown with the black line. 320260 events were detected, the histogram bin size is 80 photons. Events with low $N$ are not considered due to the detection threshold of the event registration algorithm. The red dotted line shows an exponential fit on the data $300 \leq N \leq 2000$ which was extrapolated for $0 \leq N<300$, approximating the geometrical distribution well for this high number of data points.

a FWHM of $\approx 1.37 \cdot d_{d e t}$ centred at $\mathbf{r}_{n-1}$. The algorithm is stopped if either $\mathbf{r}_{n}-\mathbf{r}_{n-1}<\epsilon$, where $\epsilon=3.6 \cdot 10^{-6} d_{d e t}$, or after 100 iterations. In the first case the position and number of photons $N$ are saved, in the latter case the corresponding event is discarded.

The localisation algorithm described above is called 'Gaussian mask fit' [50]. On the one hand it represents a computationally faster alternative to a full least squares fit of the PSF. On the other hand, it yields more accurate results than a simple centroid determination in the case of low signal to noise $(\mathrm{S} / \mathrm{N})$ ratios [51] and minimises undesired influence of adjacent spots on the localisation precision [50].

The finite pixelation of the camera represents a further loss of information in addition to the noise mentioned above, since the exact position where a photon hits the respective detector pixel is lost. Finer pixelation, however, leads to more noise due to readout and dark counts of the increased number of pixels per PSF. The optimum pixel size is about $s_{\text {det }}$ [50].

\subsubsection{Photon Statistics}

While the spatial distribution of the photons stemming from one molecule can be approximated by a Gaussian distribution, the number of photons $N$ per burst follows a geometrical distribution as will be explained in the following.

Let us assume a photoswitchable dye with an on-state A and an off-state B, where the ontransition $\mathrm{B} \rightarrow \mathrm{A}$ can be induced by light with a rate $k_{o n}\left(I_{o n}\right)$ depending on the activation light intensity $I_{o n}$, whereas the reverse reaction is of spontaneous nature with a rate $k_{o f f}$, as shown in figure 1.11(a). The dye is switched off either by spontaneous decay to state $B$, by irreversible photo-bleaching or by transferring it to another dark state such as a long-lived triplet state, from which it decays again to the ground state. For the switchable organic dyes used within this thesis it can be assumed that $k_{o f f}$ is negligibly small compared to the latter two processes under normal imaging conditions. A Jablonski diagram of the states is shown in figure 1.11 (b). For each excitation-emission cycle there exists a certain probability $p_{\text {dark }}=k_{\text {dark }} /\left(k_{\text {dark }}+k_{f l}\right)$ for the fluorophore to be destroyed or to go into a dark state 
with a lifetime $\tau_{\text {dark }}=1 / k_{\text {rec }} \gg t_{\text {frame }}$, where $t_{\text {frame }}$ is the typical camera frame time and $k_{r e c}$ the rate for the recovery to the ground state. The probability to extract $N$ photons from a molecular burst is thus $P(N)=p_{\text {dark }} \cdot\left(1-p_{\text {dark }}\right)^{N}$. The number of photons for a large number of bursts thus follows a geometrical distribution with the average photon number $\bar{N}=1 / p_{\text {dark }}$. An example of that is shown in figure 1.11 (c) for 320260 events. The differing brightness of the events can also nicely be seen in figure 1.9 , which shows the exemplary camera frame.

\subsubsection{Image Construction and Display}

SMS-microscopy relies on computational analysis in the data acquisition process and the subsequent image construction. It is therefore worthwhile to consider the influence of acquisition and data post-selection parameters on the final image. Furthermore, the actual display of the data leaves room for different approaches with their respective advantages and disadvantages.

A central aspect of SMS-microscopy is that it has to be ensured that each event stems from one marker only. Otherwise, the centre of the resulting multimarker event will be localised, leading to a wrong localisation and a loss of object information. This can be controlled during the measurement for example by ensuring that all events on a camera frame are spatially well separated. If a critical number of overlapping, that is non-circular, signal patterns is observed, the activation is too high and has to be reduced. The non-circular patterns can be discarded to reduce false localisation. For spatially extended objects, this criterion is normally sufficient. When imaging samples consisting of spatially well separated objects of subdiffraction size, such as protein clusters on a membrane, this criterion is not sufficient. If many markers within one of these objects are activated simultaneously, the signal will still be circular due to their proximity [14]. Special care has thus to be taken.

In that case, two other methods to avoid multimarker events can be applied: If an event is registered on the same spot for a consecutive number of camera frames whose added acquisition time exceeds the average on-time of the molecule under the given illumination conditions by a certain degree, it is likely to stem in fact from multiple markers and has to be discarded. Furthermore, if the brightness of the event exceeds a certain value above the average photon number per molecular burst, it is also likely to stem from multiple markers and has to be discarded as well. These two parameters, that is, maximum tolerable on-time of an event and the maximum intensity threshold, have to be determined empirically according to the properties of the marker in its environment.

Another important parameter concerning the properties and quality of the subsequent image is the lower signal threshold $T$. Increasing $T$, that is displaying only events with a high photon number, leads to a higher average photon number of the registered events and thus to a higher localisation accuracy and a better resolution according to equation 1.23 . However, this leads to a massive loss of registered events, since due to the geometric distribution of $N$, the frequency of events decreases drastically for increasing $N$, as seen in figure 
1.11(c). Therefore the image might appear spotty and details of the object might be missed if $T$ is set too high. If the staining of the sample is good enough, this can be compensated by increasing the measurement time, assuming that the brightness of the dyes is independent of spatial variables, for example due to inhomogeneities in the environment. In that case, regions favourable for the dye concerning its brightness would be overestimated.

These examples show that the parameters for the data processing have to chosen carefully.

At the present stage, two different methods of image display are established. The first one [14], called the 'Gaussian view' according to Middendorff [52] presents each registered event by a Gaussian spot with the FWHM equal to the theoretical resolution $\Delta r$ given by equation 1.23, which in turn depends on the event's photon number. The Gaussian is normalised such that the integral over the area is unity. A picture displayed in this mode can for example be seen in figure 3.9 (a) in section 3.1.6. The second method [17], named 'histogram view' [52] simply bins the events according to their position and displays the resulting twodimensional histogram in a colour coding. All events are weighted equally. An SMS-image in this mode of display can be seen for example in figure 3.5 (a) and (c). The size of the bins depends on the average lateral resolution $\Delta r$ and is typically chosen to be slightly below half that value in order to sample the image well enough and fulfill the Nyquist criterion, while still retain enough counts per bin for a statistically significant object density representation [14].

The first method attempts to merge position and accuracy information in the display of each event. That way, however, it is not obvious if an image value at a certain position originates from an actual event at or very close to that location or from an outer part of a broad 'credibility' Gaussian. It also features a broader effective imaging PSF as elucidated in detail by Middendorff [52]. The histogram method on the other hand often shows more pixelated images, especially if the image exhibits a low dynamic range.

\subsubsection{Required Marker Properties}

Summarising the preceding sections, the following requirements for a fluorescent SMSmarker can be stated:

- A high average number of photons $\bar{N}$ per burst has to be extracted in a short time to provide a high resolution at high imaging speeds.

- A good control of the activation has to be ensured, most practically by light.

Note that if initially all markers are switched off, it is sufficient to switch them on once in a controlled way, the off-transition can always be performed via photobleaching

- The ratio $N_{o n} / N_{o f f}$ of the number of molecules in the on- and off-state, respectively, has to be low. Only that way the sample can be stained densely, enabling to mark all features of the samples with a sufficient amount of markers, while still only having a sufficiently low density of activated markers, that is only one within each PSF-sized area. 
- A good signal contrast between the on- and off-state is required to ensure that no disturbing signal is registered from the high number of molecules in the dark state.

\subsection{Similarities and Differences of SMS and RESOLFT}

Throughout the preceding sections, fundamental and practical similarities and differences between the two high-resolution methods became clear. Each method has its own peculiarities which might make it more suitable for a particular application than the other. Ten important aspects will be revisited in the following.

- Same basic concept: Abbe's resolution barrier does not prevent to locate single subdiffraction-sized objects with high resolution. The challenge is to resolve subdiffraction patterns which are densely labelled with an arbitrary number of markers within the area of the PSF [11], which can be seen as a characteristic area, $A_{c}$. The key principle of both methods for breaking Abbe's diffraction barrier is to switch markers between two discernable states [11]. Both methods use the switchability to transfer a certain subset of markers to a 'dark'-state where it does not interfere with the signal coming from a region much smaller then $A_{c}$. That way, regions of subdiffraction size can be imaged individually. By sequential readout, the whole sample can be imaged.

- Inverse application of concept: The practical difference in the application of the signal switching is that in RESOLFT-microscopy the position of the signal is predefined by the scanning position and the signal of the markers in the surrounding area is switched off in a defined way to make the fluorescent volume as small as possible. On the contrary, in SMS-microscopy, the uncontrolled, stochastic switching leads to the situation that the signal already stems from the smallest possible volume, that is a single marker, but its position is unknown and has to be determined from its signal [11].

- Image acquisition: The previous point implicates that in the RESOLFT-concept the whole sample is scanned in a predefined way to obtain the signal, whereas in SMSmicroscopy the signal is collected from random positions until the image is complete. It is therefore necessary for SMS-microscopy to define a proper end-of-measurement criterion.

- Computation: Whereas RESOLFT-microscopy leads to high-resolution images by purely physical means, SMS-imaging requires computational data processing and postselection of events. This requires a careful choice of parameters in the processing in order to avoid biased images and artifacts.

- Switching fatigue: In the case of RESOLFT-microscopy the markers at each position undergo a multitude of switching processes before and after the signal from that position is collected. This is due to the controlled scanning procedure, which has to 
cover the whole sample. This number can be estimated as $\approx A_{d e p} / A_{\text {pixel }}$ times, where $A_{d e p}$ is the area of the switch-off PSF with $I(\mathbf{r}) \geq I_{\text {sat }}$ and $A_{\text {pixel }}$ the area of a scan pixel. It can be on the order of 1000 for high resolution images. This fact represents a bottleneck for some RESOLFT-microscopy applications at the present stage, since switching concepts such as Ground State Depletion (GSD), photoswitchable proteins and also the photoswitchable organic compounds used in this thesis show a considerable switching fatigue. SMS avoids this problem by the random activation scheme, where it is sufficient to switch on each marker only once [11].

- Acquisition time: For typical RESOLFT-microscopes using a single scanning focus and assuming a square region of interest (ROI) the total image acquisition time is $t_{t o t}=t_{0} \cdot\left(a_{R O I} / a_{\text {pix }}\right)^{2}$. Here, $t_{0}$ is the lowest possible pixel dwell time where the collected signal is still sufficient and $a_{R O I}$ and $a_{p i x}$ are the side lengths of the ROI and the pixel, respectively. Small regions can be imaged with high speed, even video rate and beyond [54, 55]. Large ROIs, however, will take a rather long time, due to the quadratic time scaling. In the SMS case, interestingly, the measurement time depends on the marker density distribution. Only one event per $A_{c}$ can be measured per frame. This highest possible activation density is reached for that $A_{c}$-sized region which shows the highest marker density. It therefore defines the maximum applicable activation light intensity for the whole field of view and thus the measurement time.

- Resolution: In the case of RESOLFT the maximum applicable switch-off light intensity defines the actual resolution [45]. For SMS-microscopy, the average photon number $\bar{N}$ of all registered events is the critical variable [49, 50, 51]. For a given marker, $\bar{N}$ can be increased by increasing the threshold $T$ for the minimum photon number. This reduces the number of registered events, but it can be compensated by increasing the measurement time [52], assuming a sample where differences in the environment do not have any influence on the marker brightness. Thus, for sufficiently high marker density, the resolution of SMS can be increased by increasing the measurement time.

- Influence of background light: Since SMS-microscopy is based on single molecule localisation, a low background signal is mandatory for imaging. The ensemble-based RESOLFT-method, which is additionally usually combined with confocal detection, should be more adequate for imaging objects which bring about higher background signal [11].

- Influence of noise: Residual background- and readout-noise noise is efficiently filtered in SMS-microscopy, since it applies an event detection threshold. Areas with no dye yield no signal at all in the final image. Images therefore appear usually very 'clean'. Background- and readout-noise only lead to reduced localisation precision, not to overall background signal. In RESOLFT-microscopy, every registered photon and the readout noise are displayed in the image. Therefore, these images do show background signal also in unstained regions.

- Parallelisation: Most RESOLFT-applications up to now use only single beam setups. Although parallelisation using multiple beam configurations or line scanning is possi- 
ble [39, 40, 8, 56], it also represents a higher experimental effort. Due to the use of a camera, SMS-microscopy features per se an efficient parallelisation. Using a detector chip with a larger number of pixels increases the field of view without influencing the data acquisition time, as long as the chip readout-time is not the limiting factor. 


\section{RESOLFT-Microscopy with Photoswitchable Organic Dyes}

This chapter covers the main results of the work concerning RESOLFT-type high resolution microscopy which was performed in the context of this thesis.

As has already been discussed in section 1.4, RESOLFT high-resolution microscopy makes use of a mechanism to repeatedly switch off and reactivate fluorescence in certain regions of the sample in order to gain an increase in resolution beyond the diffraction limit. In the case of its hitherto most successful principle, STED-microscopy, the switching-off is performed by stimulated emission of the excited dye molecules [12, 41]. The lifetime $\tau$ of the excited state of a typical organic dye is $\approx 1 \mathrm{~ns}$, and the typical cross section $\sigma$ for stimulated emission is $\approx 10^{-16} \mathrm{~cm}^{2}$ as for the commercial marker Atto647N in $\mathrm{H}_{2} \mathrm{O}$ at $750 \mathrm{~nm}$ [57]. We can calculate $I_{\text {sat }}$ using equation (1.15), where in this special case $t_{0}=\tau$, since the de-excitation has to be performed within the lifetime of the fluorophore, and $\sigma_{A B}=\sigma$. Equation (1.15) then yields almost $7 \cdot 10^{25}$ photons $/\left(\mathrm{s} \cdot \mathrm{cm}^{2}\right)$ or in other words $I_{s a t} \approx 2 \mathrm{MW} / \mathrm{cm}^{2}$. The values for actual imaging may easily exceed $I_{\text {sat }}$ by a hundredfold in order to obtain sufficient fluorescence switch-off also in those regions where the switch-off laser beam does not have very high relative intensity [44].

These high laser intensities may imply problems such as photobleaching of the dyes and harming cellular objects in case of live cell imaging. Additionally, at least at the time of the planning and execution of the experiments presented here, STED-microscopy was performed with expensive, pulsed high-power lasers to obtain a sufficiently high intensity in the switch-off beam at the right wavelengths. Although with the recent introduction of CWSTED, using continuous wave (cw) lasers [58], and the use of super continuous light sources [59], STED-setups have become much more convenient and also cheaper, the problems such as photodamage still remain. Therefore it is worthwhile to look for other switching mechanisms.

\subsection{The Switching Mechanism}

Promising candidates for RESOLFT-microscopy with low light intensities are photoswitchable fluorescent proteins and organic compounds [39, 13]. Using light of different wavelengths, these substances can be switched between two discernable states and back, where at least one of these states is fluorescent. The underlying process is a photoinduced rearrangement of the atoms (isomerism). The 'on' and 'off' isomeric states have typical lifetimes of $\tau \gg 1 \mathrm{~ms}$ instead of the nanosecond range in case of STED. This optical bistability is very valuable, since the light-induced switch-off reaction does not have to compete with the 
spontaneous decay to the off-state. In other words, the intensity of the switch-off light is no longer governed by the fact that the switch-off has to take place during the extremely short lifetime of the fluorescent state but by the maximum tolerable pixel dwell time of the measurement, which is usually in the range of $10 \mu \mathrm{s}-1 \mathrm{~ms}$. Therefore, it leads to the fact that $I_{\text {sat }}$ can be more than $3-4$ orders of magnitude lower as compared to STED.

Previous experiments by Hofmann et. al. [13] using photoswitchable proteins showed that it is indeed possible to break the diffraction barrier with only a few $\mathrm{W} / \mathrm{cm}^{2}$ of illumination intensity. In this work, organic photoswitchable compounds were used in a similar approach [21].

Unfortunately, organic dyes which are both reversibly photoswitchable and fluorescent are very rare since the relocation of atomic groups is antagonistic to efficient fluorescence emission. The relocation processes typically occur on a picosecond time scale [31] and are thus predominant. Most photoswitchable dyes just show a different absorption spectrum for each isomer, an effect called 'photochromism', but do not fluoresce in either isomeric state. Fluorescence, however, is desired for efficient signal detection, as discussed in section 1.1. This problem was circumvented by using a non-fluorescent photochromic compound as an acceptor for Förster Resonance Energy Transfer (FRET or RET) [60, 61] and a non-photochromic fluorescent dye as a donor [62, 63].

RET is a non-radiative dipole-dipole energy transfer between two chromophores. It strongly depends on the distance $r$ between the two dyes. Its efficiency, defined as the fraction of photons absorbed by the donor that are transferred to the acceptor, is given as $E_{R E T}=$ $R_{0}^{6} /\left(R_{0}^{6}+r^{6}\right) . R_{0}$ is the characteristic Förster distance, at which $E_{R E T}=0.5$.

The fluorescent donor dye has been chosen such that its emission spectrum overlaps with the absorption spectrum of only one of the isomers of the acceptor. In this 'off'-state, RET will take place between the excited donor and the acceptor, if they are sufficiently close. The excitation energy of the donor is thus transferred to the photochromic acceptor which cannot fluoresce but undergoes de-excitation by radiationless decay. Therefore, the fluorescence of the donor is effectively switched off. If the acceptor is in the other isomeric state, the 'on'state, no RET will occur, since now the acceptor does not absorb any more in the spectral region of the donor emission. The donor can now fluoresce. The combination of these dyes therefore forms a photoswitchable, fluorescent system.

In a first experiment, which is described in the following section 2.2.1, a mixture of the two dyes embedded in a polymer was used to demonstrate the functionality of this concept [64]. The most general solution to obtain such a photoswitchable dye pair, however, is by covalently linking the two molecules [62, 63]. In that state, this dyad can be used to conveniently stain samples. Section 2.2.1 shows the results of a study towards that direction. 


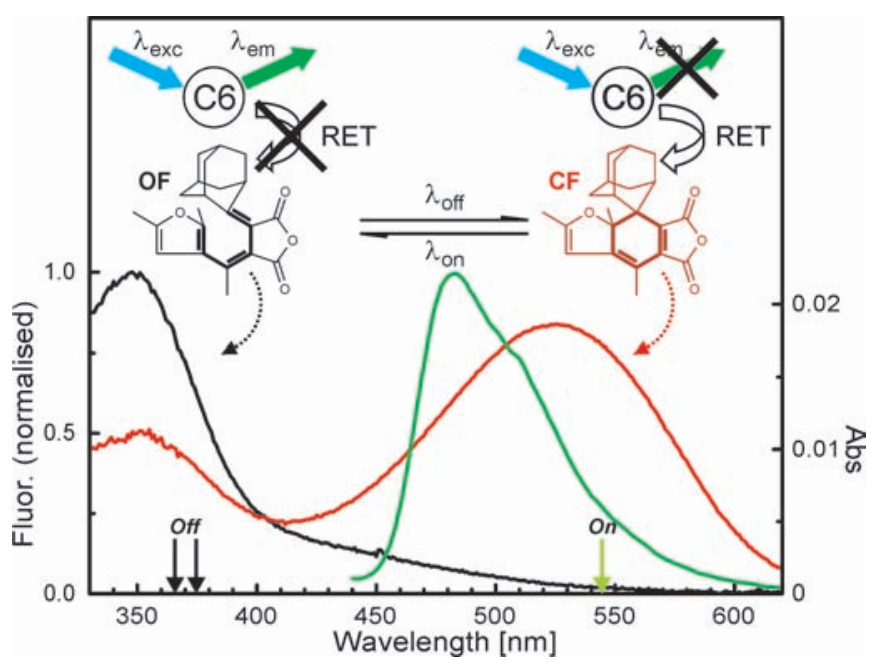

Figure 2.1: Principles of the fluorescence switching mechanism. The upper part of the figure shows the structures of the open form (OF) and closed form (CF), that is the two isomers, of Ab670 in black and red, respectively. The lower part shows the absorption spectra of the OF in black and of the photostationary state of Ab670 after irradiation with light of $\lambda_{\text {off }}=366 \mathrm{~nm}$ with a red line. In this case the ratio $\mathrm{OF}: \mathrm{CF}=25: 75$. The emission spectrum of $\mathrm{C} 6$ is shown with the green line. The sketch in the upper part visualises that fluorescence resonance energy transfer (RET) does only take place when Ab670 is in the CF. Then C6 does not fluoresce. If Ab670 is in the OF, no RET takes place and C6 can emit light. The Ab670 can be switched from OF to CF with near-UV light, for example $\lambda_{o f f}=366 \mathrm{~nm}$ or $375 \mathrm{~nm}$ as in the later experiments, and back with green light, here with $\lambda_{o n}=543 \mathrm{~nm}$.

\subsection{RESOLFT-Microscopy with a Mixture of Photochromic and Fluorescent Dyes}

\subsubsection{Properties and Kinetics of the Photochrome-Fluorophore System}

In this study [21], the photochromic compound Aberchrome 670 (Ab670) which belongs to the furyl-fulgides family, was used as the acceptor. The fluorescent donor dye was Coumarine 6 (C6). The furyl-fulgides are not affected by thermal isomerisation processes, so that this photochromic dye is bistable, leading to a dark state lifetime $\tau$ much larger than the timescales of a typical imaging procedure. The forward and back reaction between the two isomers is only induced by light, which makes it very suitable as a switch for RESOLFTmicroscopy. The working principle of the switching via RET is shown in Figure 2.1. The upper part of the figure shows the structure of Ab670 in its open form (OF, black structure) and the closed form, (CF, red structure). As depicted in the scheme, RET is not possible in the OF and the C6 can thus fluoresce when excited with $\lambda_{e x c}=415 \mathrm{~nm}$ in our case. In the CF of Ab670, RET can take place and the fluorescence of a nearby C6 is switched off. The lower part of the figure shows the absorption spectra of the acceptor Ab670 embedded in a poly(methyl methacrylate) (PMMA) polymer-film. The absorption spectrum of the OF is shown with a black line and the absorption spectrum of Ab670 in its photostationary state 


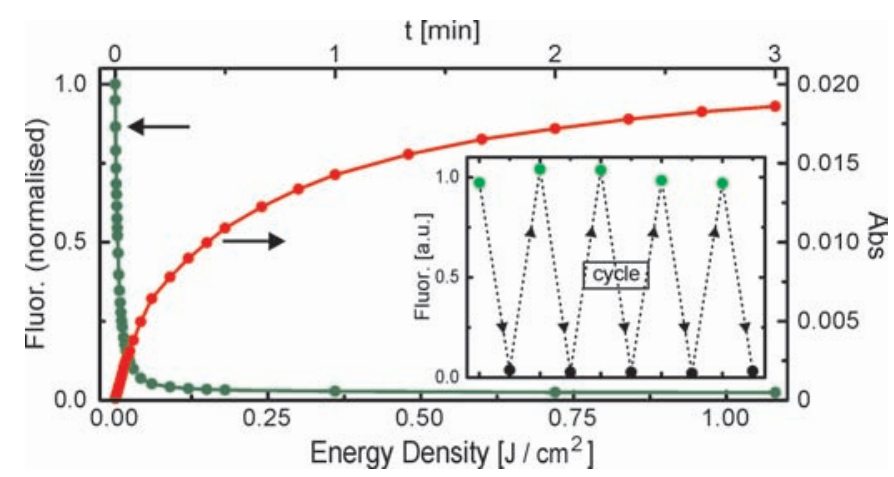

Figure 2.2: Fluorescence and Absorption of a PMMA film doped with C6 and Ab670 under irradiation with $366 \mathrm{~nm}$ light. The green graph (left axis) shows the normalised emission of C6 at its maximum at $485 \mathrm{~nm}$, the red one (right axis) the absorption of the CF of Ab670 at $522 \mathrm{~nm}$. The inset shows the fluorescence signal for five consecutive irradiation cycles after applying near-UV light (violet symbols) and green light (green symbols).

after irradiation with $366 \mathrm{~nm}$ with a red line. In the latter case, the conversion from the initial OF to $\mathrm{CF}$ was calculated to be $\approx 75 \%$ from absorption measurements and high performance liquid chromatography (HPLC). One can easily see that now, with a substantial part of the Ab670 molecules being in the $\mathrm{CF}$, the emission spectrum of C6 (green line) overlaps significantly with the absorption spectrum of Ab670, thus leading to RET. The absorption spectrum of the OF of Ab670 on the other hand shows a much smaller overlap with the emission spectrum of $\mathrm{C} 6$, which can then fluoresce efficiently. While the switching reaction from $\mathrm{OF}$ to $\mathrm{CF}$, the 'off' reaction, is induced by near-UV light with a wavelength $\lambda_{\text {off }}<380 \mathrm{~nm}$, the back reaction ('on') is driven by green light around $550 \mathrm{~nm}$. At these wavelength ranges, the difference between the two spectra is largest, leading to the best switching ratios. In order to minimise the disturbance of the ratio of $\mathrm{OF} / \mathrm{CF}$ while reading out the fluorescence of C6, the fluorophore was always excited at the isosbestic point of the photochromic system ( $405-415 \mathrm{~nm}$ ), that is the wavelength range where the absorption of the OF and the CF is the same.

The performance of this photochromic-fluorophore system concerning switch-off efficiency and reversibility was tested with PMMA films containing a mixture with 0.33 and $33 \mathrm{mmol} / \mathrm{kg}$ of polymer of C6 and Ab670, respectively. The massive excess of Ab670 was necessary to provide a sufficient amount of RET acceptors in the CF within the Förster radius of each C6 molecule even after photobleaching of Ab670 in the course of the measurement. The Förster radius for this system is $36 \AA$ [21]. The films were irradiated with near-UV and visible light from a mercury lamp. Figure 2.2 shows the kinetics of the fluorescence switchoff process. In the large plot the fluorescence of C6 at its emission maximum at $485 \mathrm{~nm}$ is plotted with a green line against the energy density of the previously applied switch-off irradiation at $366 \mathrm{~nm}$. The signal is normalised to the fluorescence of the fully activated photochromic-fluorophore system. The absorption of the system at $522 \mathrm{~nm}$ is shown with a red line. The latter directly represents the concentration of the CF. 
The increase of the concentration of Ab670 molecules in the CF is much slower than the decay of the fluorescence signal, which shows a decrease much faster than the mono-exponential decay of the model in equation 1.14 . This is due to the fact that each donor has multiple acceptors within the Förster distance. Furthermore, it can be seen from the plot that the fluorescence switch-off is almost complete.

The reversibility of the system has been tested by repeated irradiation with $366 \mathrm{~nm}$ switchoff light and $543 \mathrm{~nm}$ activation light and intermediate probing of the fluorescence with the $415 \mathrm{~nm}$ excitation light. The inset in figure 2.2 shows the result of this measurement. The green circles denote the fluorescence signal after irradiation with the activating light and the black circles the signal after irradiation with the switch-off light. The fluorescence signal was normalised to the average of the signal in the activated state. Several consecutive cycles are shown with no sign of switching fatigue.

These measurements show that this system has a very good switch-off efficiency and is reversibly photoswitchable, therefore it is indeed a potential candidate for RESOLFT-type subdiffraction imaging.

\subsubsection{Microscope Setup and Imaging Results}

The microscope used to demonstrate the applicability of this dye system for RESOLFTsubdiffraction imaging was a custom built setup based on the scanning microscopy principles discussed in section 1.3. A scheme of the microscope is shown in figure 2.3 (a). In this case, only a one dimensional resolution enhancement was applied, the direction is defined as $x$ in our coordinate system. Three lasers were used for the imaging. The light for excitation of C6, $\lambda_{e x c}=415 \mathrm{~nm}$ was provided by a laser diode (DL100, Toptica, Gräfelfing, Germany). A He-Ne laser (25LGP193-230, Melles Griot, Carlsbad, CA) was used to obtain the reactivation light of $\lambda_{\text {on }}=543 \mathrm{~nm}$. The photochromic-fluorophore system was switched off with light of $\lambda_{o f f}=375 \mathrm{~nm}$ from a laser diode (iPulse-375, Toptica). All beams were linearly polarised perpendicularly to the direction of resolution enhancement, that is in $y$-direction, and mode cleaned by a pinhole (not shown in the figure) to obtain a $\mathrm{TEM}_{00}$ mode. The beams were expanded such that their waists were larger than the aperture of the objective (PL APO 100x, 1.4-0.7, Leica Microsystems, Wetzlar, Germany), collimated and coupled into the same beam path using dichroic mirrors DM1, DM2 and DM3 in figure 2.3 (a). A spatial light modulator (SLM, X8267-3558, Hamamatsu Photonics K K, Hamamatsu City, Japan) was additionally placed in the path of the $375 \mathrm{~nm}$ beam. This device allows one to impose a variable phase shift onto the wave front of the beam. Here, a $\pi$-phase shift was applied between the two halves of the beam so that it features a node in the $x y$-plane of the focus, as discussed in section 1.4.2. Figure 2.3 (b) shows the central plane of the PSF of the excitation beam measured by scanning a reflective silver bead through the excitation spot (top) and of the switch-off beam (bottom, calculated).

The fluorescence light was collected by the same objective, decoupled from the excitation beam path by DM3 and further cleaned by a suitable fluorescence filter F. To obtain confocalisation, it was then focussed into an optical fibre of $62.5 \mu \mathrm{m}$ core diameter, serving as a pinhole (fibre not shown in figure 2.3 (a)). The diameter corresponds to 1.4 times the size of the backprojected Airy disk (defined as the distance between the first two minima). The 

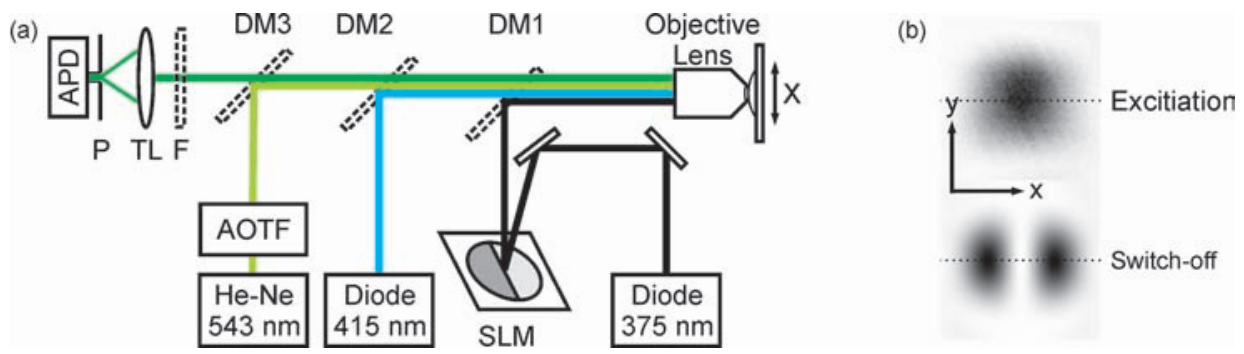

Figure 2.3: (a) Schematic sketch of the microscope setup. For a clarity, only the beam pathes are shown, not the actual beam size and shape. DM1-3: dichroic mirrors, SLM: spatial light modulator, AOTF: acousto optic tunable filter, F: fluorescence filters, TL: tube lens, P: pinhole, APD: avalanche photo diode. (b) Central $x y$-plane of excitation PSF (top) and switch-off PSF (bottom).

fibre transferred the light to an avalanche photo diode (APD) which was used as detector. The APD was gated simultaneously with the excitation laser. The light intensity from the laser diodes could be directly controlled, the excitation light was modulated with an acousto optic tunable filter (AOTF, AA.AOTF.nC-TN1003, AA Optoelectronic, St. Remy, France). A piezo scanning stage was used to move the sample.

An image is acquired by scanning the sample pixel by pixel and reading out the fluorescence signal. A superresolution image and a confocal image for comparison are recorded simultaneously. The temporal succession of the three laser pulses was controlled by a programmable pulse generator (9500 Series, Quantum Composers, Bozeman, MT, USA). A typical laser pulse sequence for each pixel of the experiment is as follows. First, a pulse is sent in with $\lambda_{\text {on }}$ to fully activate the focal region. The second pulse is an excitation pulse with $\lambda_{\text {exc }}$, which gives the signal for the confocal image. Thirdly, a switch-off pulse with $\lambda_{o f f}$ is applied. Now, RET is possible for almost all C6 molecules in the focal region except for those in the nodal plane of the depleting PSF. Therefore, the signal collected with the fourth pulse, which is again with $\lambda_{e x c}$, stems only from the effective PSF $h_{e f f}(x, y, z)$, which is a plane perpendicular to the $x y$-plane and parallel to $y$ which is much thinner than the excitation PSF $h_{\text {exc }}$.

In order to estimate the resolution of the microscope, grooves etched into a glass cover slip by ion beam etching (Fraunhofer Institute IISB, Erlangen, Germany) were used which were $\approx 8 \mu \mathrm{m}$ long, $600 \mathrm{~nm}$ deep and $90 \mathrm{~nm}$ wide. The dye-doped PMMA film was spincoated onto the sample so that primarily the bottom part of the grooves was covered. Figure 2.4 (a) shows the intensity profile of a line scan of a single groove filled with the doped PMMA with the full red line. For this measurement, the pulse energy density for the switchoff PSF $h_{\text {off }}$ was $0.05 \mathrm{~J} / \mathrm{cm}^{2}$ on average in the focal plane. The FWHM reads $112 \mathrm{~nm}$ as compared to the FWHM of the confocal excitation PSF $h_{\text {exc }}$ of $230 \mathrm{~nm}$, which represents the resolution of the conventional confocal microscope and which is shown with the blue line. A significant resolution increase is thus obvious.

The width of the line scan of the single groove, however, does not represent the full resolution of the microscope, since it is a convolution of $h_{e f f}$ and the object, as stated by equation 1.8 . The influence of the PSF of the detection beam path can be ignored in our case due to the 

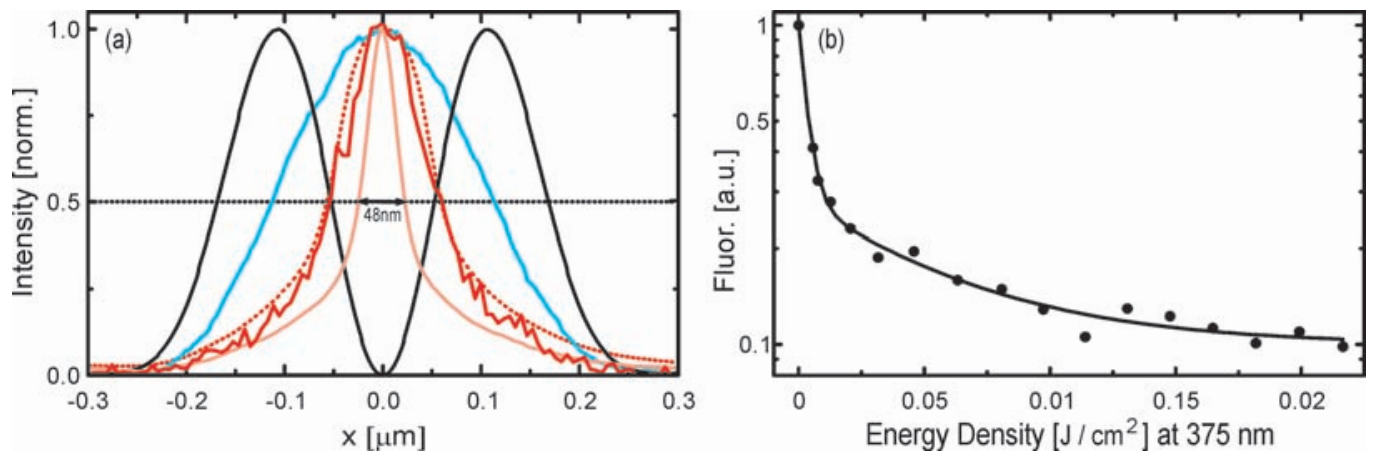

Figure 2.4: (a) Central line profile of $h_{\text {eff }}$ (light red), $h_{\text {exc }}$ (blue) and $h_{o f f}$ (black). The full red line shows a line scan of a single groove in high resolution mode. The dotted red line shows the expected shape of the line scan calculated by a convolution of $h_{e f f}$ and the known object shape. Both profiles show good agreement. (b) Switch-off of the fluorescence with increasing light intensity $I_{o f f}$. irradiation. The measured data is shown with black circles, the black line is a multi-exponential fit to the data.

relatively large pinhole that was used.

The actual effective PSF $h_{e f f}$ for a switch-off irradiation of pulse length $t_{i r r}$ and intensity $I_{\text {off }}$ can be calculated as

$$
h_{e f f}(x, y)=h_{e x c}(x, y) \cdot \eta\left(h_{o f f}(x, y) \cdot I_{o f f} \cdot t_{i r r}\right),
$$

according to equation 1.16 , where $\eta$ is the switch-off function denoting the residual fluorescence, which depends on the energy density of the pulse, that is $I_{\text {off }} \cdot t_{i r r}$. Figure 2.4(b) shows the switch-off of the fluorescence signal in a single groove with increasing irradiation energy density of the switch-off pulse with black circles. The fitted black line can serve as a calibration for $\eta$. This data has been measured by overlapping $h_{e x c}$ with the switch-off PSF without the phase imprint being applied. One can see that the switch-off efficiency is not as good as in the case of a thin film (figure 2.2). This is probably due to the fact that residual fluorescence is collected which stems from regions outside the central plane since the switch-off PSF is smaller than $h_{e x c}$ due to the shorter wavelength. Decreasing the pinhole size slightly reduced this effect but on the cost of the signal to noise ratio. Figure 2.4(a) shows the central line profile of the calculated effective PSF $h_{e f f}(x, 0)$ in $x$-direction with the light red solid line for a switch-off light pulse with an average energy density of $0.05 \mathrm{~J} / \mathrm{cm}^{2}$, as in the measurement. For the calculation, the central beam profiles of $h_{e x c}$ and $h_{o f f}$ have been used (dotted lines in figure 2.4(b)) which are plotted in figure 2.4(b) with the blue solid line for $h_{e x c}(x, 0)$ and the black line for $h_{o f f}(x, 0)$. It can be read off from figure 2.4(b), that the expected resolution is $\approx 50 \mathrm{~nm}$.

The red dotted line in figure 2.4 (a) shows the convolution of the object, known from electron microscopy, and the calculated $h_{e f f}$. Good agreement can be observed between the measured and the calculated data, showing that the resolution of this system was $\approx 50 \mathrm{~nm}$ in $x$-direction. Note that the broad shoulders of $h_{\text {eff }}$ are a result of the imperfect switch-off of the fluorescence (only to about $20 \%$ at the applied $I_{\text {off }}$ per pulse) due to the above mentioned residual fluorescence. Although a higher $I_{o f f}$ would further decrease this value and enhance the resolution, it also leads to more photodamage of Ab670 and thus to decreased switch-off 

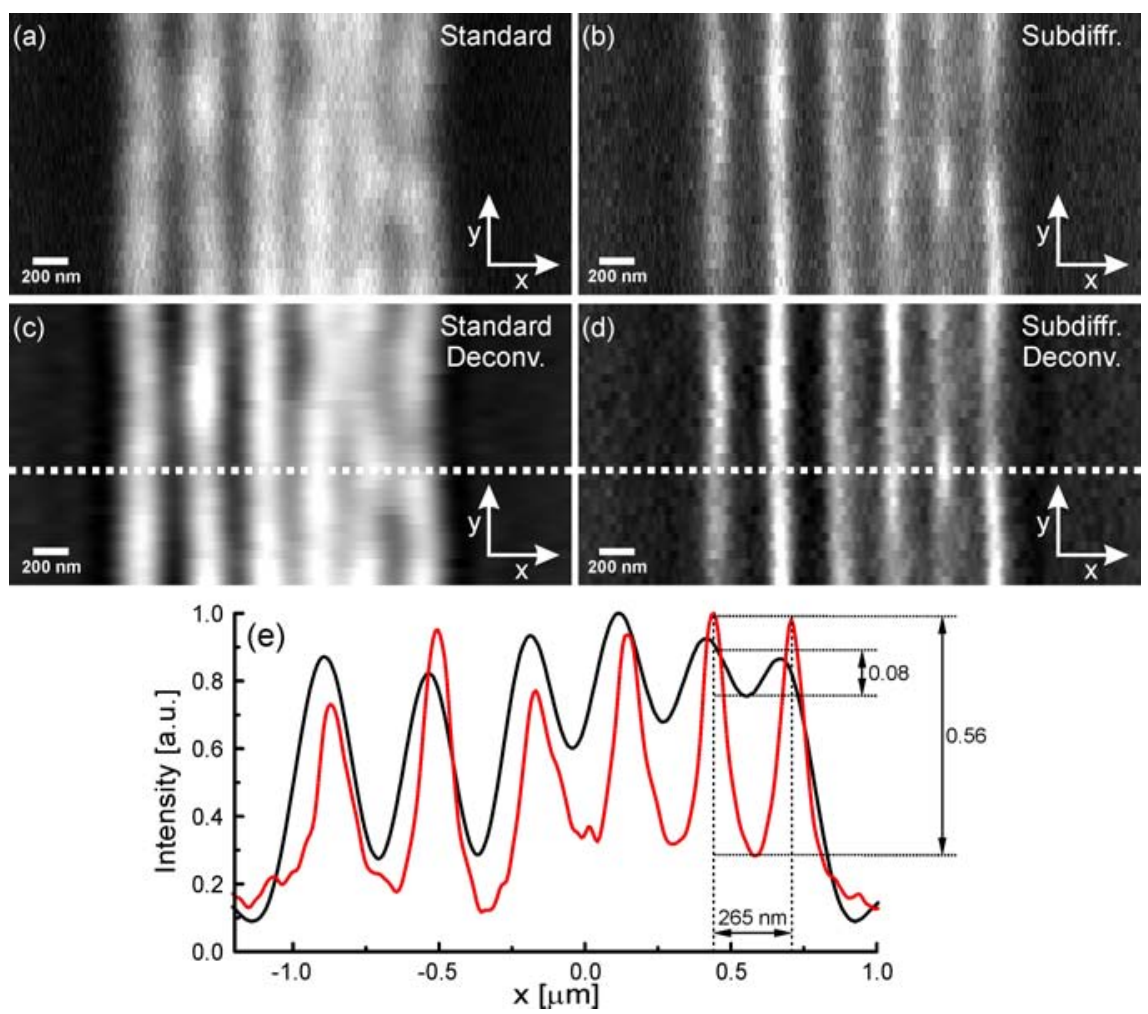

Figure 2.5: Standard confocal (a) and high resolution (b) image of set of grooves with a width of $90 \mathrm{~nm}$ and decreasing separation distance from left to right. (c) and (d) show the corresponding linearly deconvolved images. (e) depicts the line profile along the dotted lines in (c) and (d) in black and red, respectively.

efficiency with increasing number of switching cycles, as more and more Ab670 molecules are destroyed.

Figure 2.5 shows a two-dimensional image of a set of grooves with decreasing groove separation from left to right. Comparing figure 2.5(a), showing the standard confocal image and the subdiffraction image in figure 2.5(b), the difference in resolution becomes very obvious. After applying a linear deconvolution with the calculated $h_{e f f}$, which removes the effect of the broad shoulders of $h_{e f f}$, the difference becomes even more obvious, as can be seen in figure 2.5(d). For fair comparison, the classical confocal image of figure 2.5(a) was also deconvolved with $h_{e x c}$ and is shown in figure 2.5(c). A line profile along the dotted lines in figure 2.5(c) and (d) is displayed in figure 2.5(e). The increase in contrast, defined as $\left(I_{\text {peak }}-I_{\text {valley }}\right) /\left(I_{\text {peak }}+I_{\text {valley }}\right)$, where $I_{\text {peak }}$ is the average of two adjacent peaks and $I_{\text {valley }}$ is the signal of the valley in between, is obvious. For the confocal case it amounts to only 0.08 in comparison to 0.56 for the superresolved image. The power of the switch-off laser beam was $75 \mathrm{nW}$, which amounts to an intensity of $\approx 185 \mathrm{~W} / \mathrm{cm}^{2}$ in the focal plane. The powers of excitation laser and reactivating laser were $8 \mathrm{nW}$ and $16 \mu \mathrm{W}$, respectively. The pixel dwell time was set to $1 \mathrm{~ms}$ to keep the total measurement time in the range of a minute. The illumination sequence described above was thus performed within that dwell time. This 
time can, however, be easily sped up to the microsecond range by scaling up the laser powers correspondingly, which still would require only a few $\mathrm{kW} / \mathrm{cm}^{2}$ of intensity.

As a control experiment, the phase-shift pattern on the SLM was removed, transforming the $h_{\text {off }}$ into the normal focal spot. As expected, almost all fluorescence was quenched now and no resolution increase could be observed. A further control was made by turning the sample by $90^{\circ}$ so that $h_{\text {eff }}$ was squeezed in the direction parallel to the grooves. Again no resolution increase could be observed.

\subsubsection{Summary}

This experiment demonstrates that the diffraction barrier can be broken using organic photochromic dyes as the switching elements for RESOLFT-type high resolution microscopy. An advantage of this system as compared to STED is the very low laser intensities needed. This also makes the parallelization of image acquisition more easy, for example by applying arrays of holes or lines for simultaneous readout of many parts of the image.

Two major disadvantages still remain for this system. First, the number of switching cycles of the photochromic compound is limited. As already mentioned in section 1.4, twodimensional high resolution images would require on the order of 1000 cycles, which is not possible under the applied measurement conditions.

Second, for a more general application, it is necessary to covalently link the RET donor and acceptor. Only in that way a convenient staining of samples is possible. In the framework of this thesis, this problem was tackled using an alternative choice of dyes. This project and its results are described in the following section.

\subsection{Photoswitching and Imaging of Organic Fluorescent Switches in Silica Nanoparticles}

\subsubsection{Properties of the Covalently Bound Photochrome-Fluorophore System}

Since a loose mixture of dyes is impractical for staining, for example, cellular objects, the use of photoswitchable organic dye compounds was studied in this thesis where the photochromic unit and the fluorescing part are covalently linked [22]. Prior work by Bossi et. al. [65] already showed that it is indeed possible to synthesise such compounds based on Rhodamine-101 (Rh101) as the fluorophore and a photochromic diheteroarylethene (DAE). DAEs are known to suffer only little switching fatigue [62] and are also bistable. A similar compound was used in this study. However, in contrast to the one presented by Bossi et. al. [65], it has an additional amino reactive NHS-ester to enable coupling to specific structures. Figure 2.6 shows the structure of the two isomers of this compound, which is called Rh-AADAE. The fluorescent dye is Rh101 (red shaded area) which is covalently bound to the photochromic unit via a link containing an NHS-ester. The fluorophore is excited by green light, $\lambda_{e x c}=543 \mathrm{~nm}$ in this case, and emits with an emission maximum at $\lambda_{e m}=603-610 \mathrm{~nm}$, 


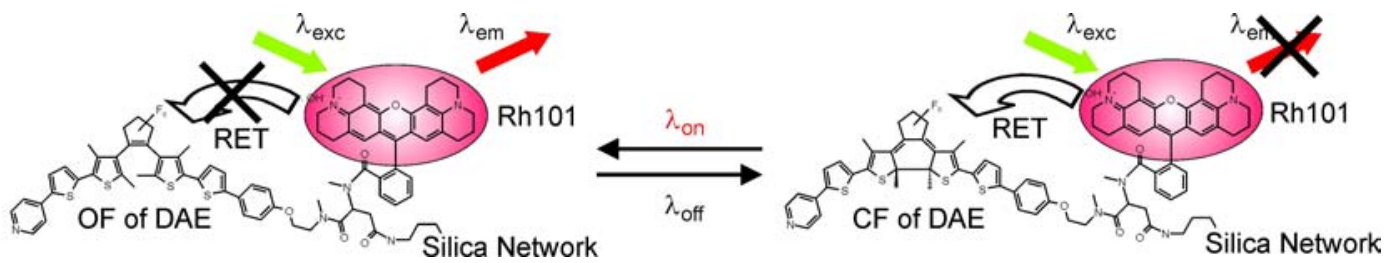

Figure 2.6: Photoswitching of Rh-AA-DAE. The open form (OF) of the DAE does not allow RET with Rh101 (marked red), therefore the compound is fluorescent, as depicted in the upper sketch. The closed form (CF) in the lower scheme shows an absorption spectrum which allows RET, therefore the compound is non fluorescent. The 'off' reaction is initiated with $\lambda_{o f f}$ in the near-UV range, the 'on' reaction with $\lambda_{o n}$ in the red spectral range. The compound can be excited with $\lambda_{e x c}$ in the green spectral region (see text).

\begin{tabular}{|cccccccc|}
\hline Compound & \multicolumn{2}{c}{$\lambda_{\max }[\mathrm{nm}]$} & $\phi_{\text {fluo }}$ & $a_{P S}$ & FM & \multicolumn{2}{c|}{$E_{R E T}$} \\
& Abs. & Em. & & & & on-state $(\mathrm{OF})$ & off-state $(\mathrm{CF})$ \\
\hline Rh-AA-DAE & 585 & 604 & 0.52 & $0.98^{[a]}$ & $94 \%$ & 0.46 & 0.98 \\
Nanoparticles & 590 & 610 & 0.52 & $0.59^{[b]}$ & $94 \%$ & - & - \\
\hline
\end{tabular}

Table 2.1: Properties of Rh-AA-DAE in ethanolic solution (first row) and embedded in silica nanoparticles (second row) in ethanolic suspension. The table shows the maxima of emission and absorption spectrum $\lambda_{\max }$ Abs. and Em., respectively, the fluorescence quantum yield $\phi_{\text {fluo }}$, conversion to CF in photostationary state $a_{P S}$, fluorescence modulation FM (see text) and RET-efficiencies $E_{R E T}$ of both isomers in the respective environment. ${ }^{[a]}$ From HPLC measurements, ${ }^{[b]}$ from absorption measurements, assuming the same absorption coefficients in silica and in solution.

depending on the environment. Again, the two isomers of the DAE show different absorption spectra, where only one of them overlaps with the emission spectrum of Rh101. If the DAE is in its OF, the spectra do not overlap, no RET can occur and the excited Rh101 can release its energy by fluorescence, as sketched in figure 2.6. In the CF, however, RET is possible and the Rh101 remains dark. The switching between the two states is performed with light in the red spectral range for the opening on-reaction and near-UV light for the closing off-reaction in the experiments.

The properties of Rh-AA-DAE in ethanolic solution are listed in table 2.1 in the first row. The calculated Förster radius for this dye pair is $50 \AA$. The RET efficiency of the linked dye is $E_{R E T}=0.98$ in the off-state. The RET efficiency in the on-state is highly reduced and only amounts to 0.46 . The conversion to the CF in the photostationary state $a_{P S}=0.98$ as measured by HPLC and is thus almost complete. This leads to a fluorescence modulation (FM) between on- and off-state of $94 \%$, where FM is defined as FM $=100 \cdot\left(1-I_{\text {fluo,off }} / I_{\text {fluo }, \text { on }}\right)$ with $I_{\text {fluo,off }}$ and $I_{f l u o, o n}$ being the fluorescence intensity of the compound in the off- and on-state, respectively, after illumination with excitation light of the same intensity in both cases. This compound therefore appears to be a very promising candidate for RESOLFTmicroscopy and other applications requiring high fluorescence modulation.

A disadvantage of this compound, however, is its insolubility in water. Direct labelling of biological samples in natural aqueous conditions therefore requires further improvement of the compound. This problem was circumvented in this work by incorporating the compound into silica nanoparticles, which are themselves hydrophilic and fully biocompatible [66, 67, 68]. 

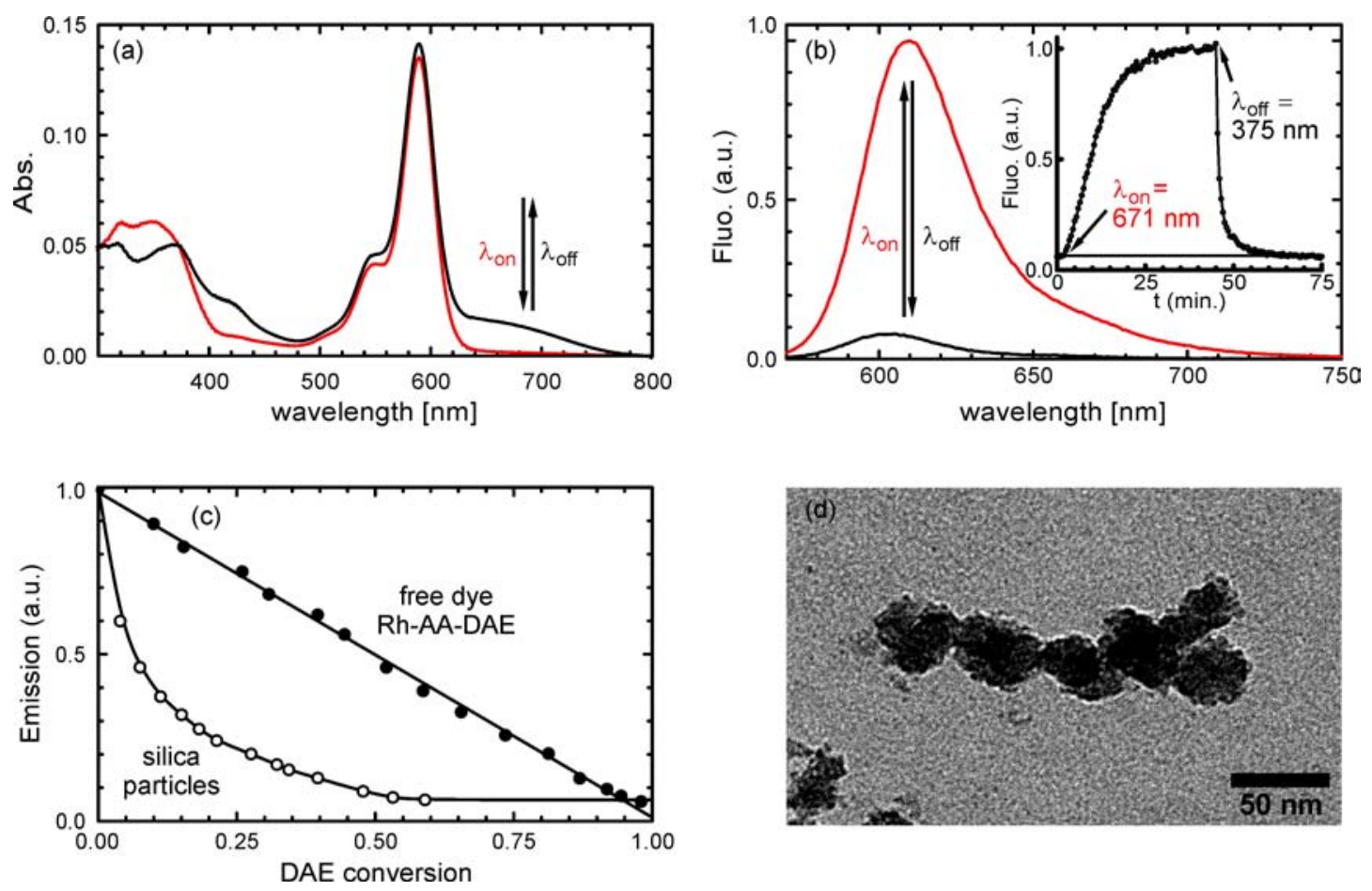

Figure 2.7: (a) Absorption spectra of the OF (red line) and the CF (black line) of Rh-AA-DAE incorporated in silica nanoparticles in ethanolic suspension. (b) Emission spectra of the OF (red line) and the CF (black line) of Rh-AA-DAE in silica nanoparticles in ethanolic suspension. The inset shows the switching kinetics of the nanoparticles in ethanolic suspension upon irradiation with $\lambda_{o n}$ and $\lambda_{o f f}$. (c) The solid black circles show the normalised fluorescence emission plotted against the conversion of the DAE for the free Rh-AA-DAE in solution. The open circles show the same for the doped nanoparticles in ethanolic suspension. (d) Electron microscope image of exemplary doped nanoparticles.

Furthermore, this strategy yields the advantage of stronger signal, the possibility of incorporating multiple markers for multiplexed encoding [69, 70] and of bestowing the nanoparticle surface with different molecular functionalities [69, 71].

The incorporation into the silica has been performed by coupling the NHS functionalised Rh-AA-DAE to (3-aminopropyl)triethoxysilane and then including the compound into the synthesis of the silica particles as described in detail in [22, 72, 73]. This way the dye is bonded to the silica network and cannot leak out. The properties of the doped nanoparticles are displayed in the second row of table 2.1. The absorption and emission maxima are redshifted by about $5 \mathrm{~nm}$ as compared to the free dye, the fluorescence quantum yield $\phi_{\text {fluo }}$ remains unchanged.

The actual absorption spectra of the two states of the doped nanoparticles can be seen in figure 2.7(a), the emission spectra in figure 2.7(b). The properties of the 'on'-state are shown with a solid red line and the ones of the 'off'-state with a solid black line. The increase of the absorption in the range between $590 \mathrm{~nm}$ and $750 \mathrm{~nm}$, which corresponds to the emission range of the Rh101-moiety, is clearly visible in figure 2.7 (a), leading to the enhanced RET efficiency and thus to a drastic decrease of the fluorescence as seen in figure 2.7(b). 
The wavelength of the activation light was chosen to be $\lambda_{\text {on }}=671 \mathrm{~nm}$ since the difference of the absorption between the off-state and the on-state is largest at this wavelength, enabling the most efficient on-transformation. The wavelength of the switch-off light was chosen to be $\lambda_{\text {off }}=375 \mathrm{~nm}$ for the same reason: at this wavelength the absorption of the on-state is much larger than the absorption of the off-state, therefore efficient conversion to the off-state is possible. At the isosbestic point of the system, at around $390 \mathrm{~nm}$, fluorescence excitation of the Rhodamine is very inefficient. In the spectral range where efficient fluorescence excitation is possible, however $(\approx 510 \mathrm{~nm}$ to $610 \mathrm{~nm})$, the absorption of the off-state is slightly higher than the one of the on-state. A certain re-activation can therefore not be avoided when probing the fluorescence. In this case an excitation wavelength of $\lambda_{\text {exc }}=543 \mathrm{~nm}$ was chosen due to the availability of a compact He-Ne laser featuring this spectral line.

The inset in figure 2.7(b) depicts the switching dynamics of the nanobeads, measured in an ethanolic suspension. The modulation of the fluorescence signal is FM $=94 \%$ as in the case of the free dye in solution. However, the actual conversion in the photostationary off-state of the nanoparticles is only $a_{P S}=0.59$, due to spatial constraints in the silica environment. The reason for the much higher fluorescence modulation is that the concentration of the compound in the beads is high enough so that intermolecular RET can occur. In other words, the distance between the molecules is small enough so that one DAE can also accept the energy from neighbouring fluorophores and not only from its own. This fact can be deduced from 2.7(c), where the emission at the emission maximum is plotted against the DAE conversion, induced by illumination with $375 \mathrm{~nm}$, for the free Rh-AA-DAE (filled circles) and the nanoparticles (open circles) in ethanolic solution and suspension, respectively. In the case of the free dye, a linear dependence is obvious. This is expected because each donor has one RET acceptor within the Förster radius, therefore the decrease of the fluorescence should be proportional to the conversion of the acceptor to the CF. In the case of the nanoparticles, a nonlinear, steep decrease is observed due to the intermolecular RET effect mentioned above. All bead measurements of table 2.1 and figure 2.7 were performed with nanoparticles of $\approx 30 \mathrm{~nm}$ diameter. An electron microscope picture of some exemplary particles is shown in figure 2.7(d).

\subsubsection{Application in RESOLFT-Microscopy}

The main goal of application of these compounds is to use them as markers for RESOLFTmicroscopy. Apart from a good switch-off efficiency, the reversibility of the switching process is of major importance. Figure 2.8 (a) shows the result of a cycling measurement on a single $30 \mathrm{~nm}$ silica particle. 30 complete cycles down to a relative intensity of $20 \%$ in the off-state could be performed by successive illumination with $375 \mathrm{~nm}$ and $671 \mathrm{~nm}$ light without any sign of fatigue. The fluorescence modulation FM of $6 \%$, which is listed in table 2.1 and shown in the inset of figure 2.7 (b), could not be reached here. The reason for that is the re-activation effect of the excitation light. The preceding measurements were taken in bead ensembles, therefore less excitation light was necessary to yield sufficient signal, leading to less re-activation.

Figure 2.8(b) shows the result of the continuation of the cycling measurement for 100 cycles, including the 30 of figure 2.8(a). Apart from photobleaching of the rhodamine fluorophore, 

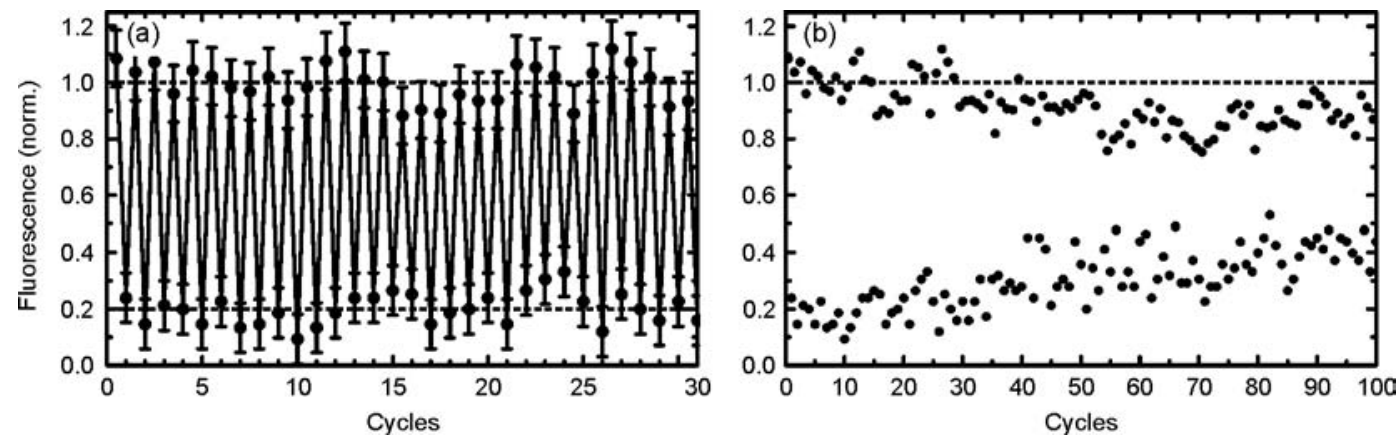

Figure 2.8: (a) Switching reversibility of Rh-AA-DAE in a single silica bead for 30 cycles. The error bars correspond to the standard deviation of the first 30 data points. (b) Switching reversibility for 100 switching cycles, including those from (a). The data is normalised to the mean value of the first 30 'on' data points in both cases.

now also the switching fatigue becomes eminent by an increase of the signal of the off-state, showing that the DAE part undergoes irreversible photodamage. The light pulse sequence for the measurement consisted of an on-pulse for $10 \mathrm{~ms}$ with an intensity of $70 \mathrm{~W} / \mathrm{cm}^{2}$, followed by a probe pulse of the excitation light with an intensity of $60 \mathrm{~W} / \mathrm{cm}^{2}$ for $2 \mathrm{~ms}$, then the off-pulse with $375 \mathrm{~nm}$ at an intensity of $210 \mathrm{~W} / \mathrm{cm}^{2}$ for $15 \mathrm{~ms}$ and finally the second probe pulse for $16 \mathrm{~ms}$ at the same intensity as the first one. The longer integration time for the second pulse was used to minimise noise when measuring the low residual fluorescence of the off-state. The sample was prepared by letting a drop of the ethanolic bead suspension evaporate on a glass coverslip and embedding the sample in standard immersion oil.

Although the relatively small number of 30 reversible cycles is by far not enough for high resolution two-dimensional images, the system was still tested using one-dimensional resolution enhancement similar to the one in section 2.2.2. The microscope setup used here resembled the setup described in that section. The same source was used for the $375 \mathrm{~nm}$ switch-off light and the $543 \mathrm{~nm}$ light was provided by the same He-Ne laser as before, only this time for excitation of the compound. For the reactivation light of $\lambda_{o n}=671 \mathrm{~nm}$, a diode pumped solid state laser (DPSSL, Monolas-671-300MM, Alphalas, Göttingen, Germany) was applied. The modulation of the phase in the off-beam was performed in the same way and using the same SLM as before. This led to a switch-off PSF $h_{\text {off }}$ with the shape shown in figure 2.9 (a) (top). The microscope objective used (ACS, 60x, Leica Microsystems, Wetzlar, Germany) had a numerical aperture of 1.3 and featured a special correction for chromatic aberration in a large spectral range.

The direction of resolution increase is again the $x$-direction. Also the pulse sequence was produced analogous to the experiments in section 2.2.2. First, an activation pulse was sent in for $2.5 \mathrm{~ms}$ with an intensity of $380 \mathrm{~kW} / \mathrm{cm}^{2}$, followed by a probe pulse of the excitation light for the conventional confocal image for $50 \mu \mathrm{s}$ at an intensity of $40 \mathrm{~W} / \mathrm{cm}^{2}$. Third the switchoff pulse was sent for $100 \mu$ s with an intensity of $500 \mathrm{~W} / \mathrm{cm}^{2}$, corresponding to $0.05 \mathrm{~J} / \mathrm{cm}^{2}$, followed by the probe pulse for the RESOLFT-image with the same settings as the one for the confocal image. 

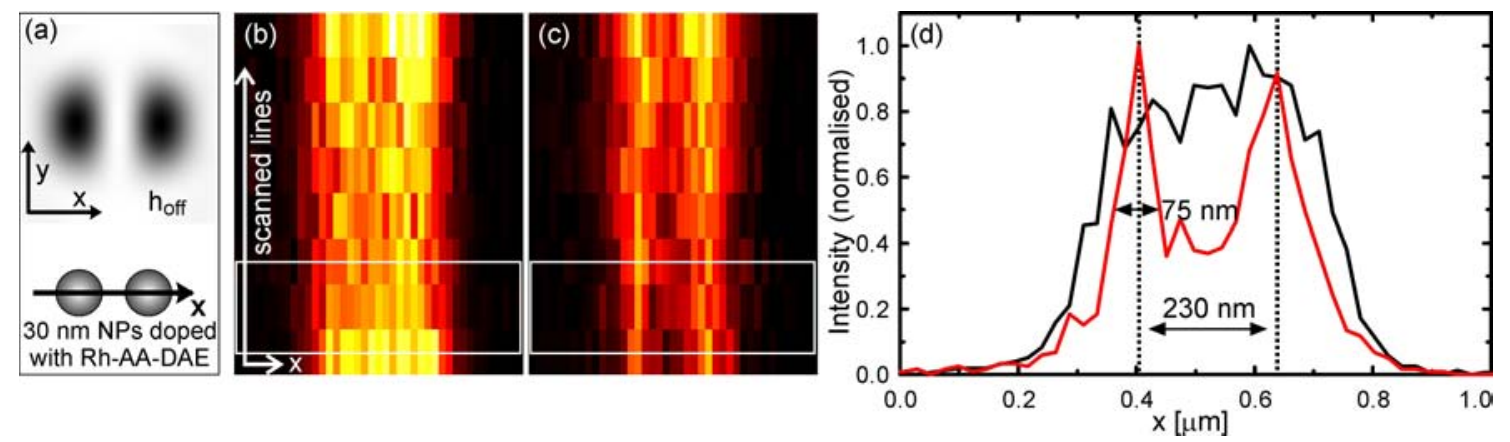

Figure 2.9: (a) Sketch of the spatial alignment of the switch-off PSF (top) and the arrangement of the sample (bottom) consisting of two adjacent beads. (b) Confocal image of eight line scans of the two adjacent beads taken in the order from bottom to top. (c) RESOLFT image of the corresponding line scans of (b). The intensity of (b) and (c) is displayed in colour-coding from black to white via red and yellow and normalised to the brightest pixel in (b). (d) Profile of the average of the first three line scans as denoted by the white boxes in (b) and (c) for the confocal image (black line) and the RESOLFT image (red line), respectively.

As a sample object, two adjacent $30 \mathrm{~nm}$ nanoparticles (NP) were chosen as shown in figure 2.9 (a) (bottom). They were scanned eight times in a line scan in $x$-direction, as shown in the figure. The resulting intensity profiles of the scans of the confocal image and the RESOLFTimage are shown colour-coded in figure 2.9 (b) and (c), respectively. The first scan is the bottom pixel line, the last scan the top line. The scanning was performed with 64 steps in $x$-direction along a distance of $1.5 \mu \mathrm{m}$, one pixel in the figure thus corresponds to $\approx 23.4 \mathrm{~nm}$ in $x$-direction. Whereas in the confocal image no specific features can be observed, the RESOLFT-image shows two clear signal peaks in the first line scans, which become less pronounced with an increasing number of scans. This double peak can be made out more clearly when averaging over the first three lines as indicated by the box in figure 2.9(c) and plotting the intensity profile in a graph, which is shown in figure 2.9 (d) with the red line. For comparison, also the averaged intensity profile of figure 2.9(b) is plotted in figure 2.9(d) with the black line. Here, no peak separation can be observed. The distance between the two beads can be read off from the plot as $230 \mathrm{~nm}$. The reason why the double peak 'fades out' with increasing number of line scans is the switching fatigue of the DAE. As more and more of the photochromic units are irreversibly damaged, the fluorescence switch-off becomes inefficient, the resolution thus becomes worse and the image more and more resembles the classical confocal image.

The resolution of the system can be determined by the size of the beads' image in figure 2.9(d). The FWHM of the left one is $\approx 75 \mathrm{~nm}$. This value compares well to measurements on other single beads and to the theoretically expected resolution. The latter has been estimated in analogy to the procedure described in section 2.2.2. The fluorescence switch-off vs. the energy density $I_{\text {off }} \cdot t_{\text {irr }}$ of the switch-off light has been determined with a single bead in the focus and is shown in figure 2.10 (a) with the black circles. The black line is an exponential fit and can be seen as the fluorescence switch-off function $\eta\left(I_{o f f} \cdot t_{i r r}\right)$. The fluorescence in a single bead can be depleted down to $\approx 15 \%$ in the actual microscope setup. Although not as good as in the ensemble measurements of figure 2.7, this value is sufficient to obtain 

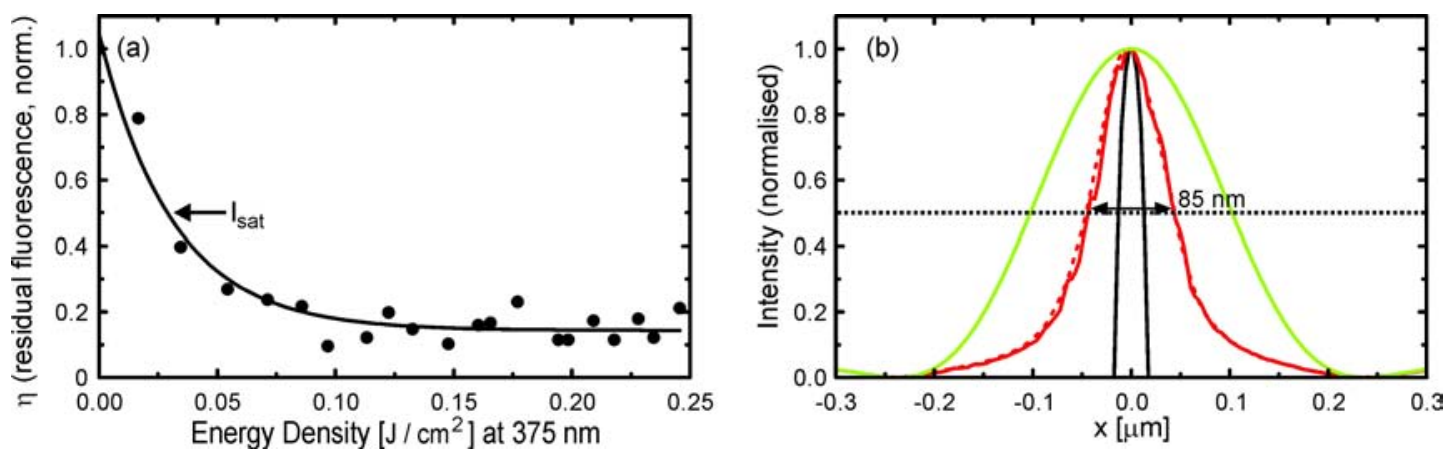

Figure 2.10: (a) Residual fluorescence $\eta$ plotted against $I_{o f f} \cdot t_{i r r}$. For the measurement, the intensity of the switch-off pulse was kept constant at $100 \mathrm{~W} / \mathrm{cm}^{2}$ and the pulse length was increased in 20 steps. Before each switch-off pulse, the bead was switched on again by an activation pulse of $2.5 \mathrm{~ms}$ with an intensity of $380 \mathrm{~kW} / \mathrm{cm}^{2}$. The probe pulse with the excitation light was shone in for $2 \mathrm{~ms}$ with $60 \mathrm{~W} / \mathrm{cm}^{2} . I_{\text {sat }}$ denotes the intensity, where the signal dropped to $50 \%$ of its original value. (b) Line profile of the calculated excitation PSF $h_{\text {exc }}$ (green line), a calculated sample object of $30 \mathrm{~nm}$ diameter (black line), the calculated effective PSF $h_{e f f}$ (red solid line) and the convolution of the object with $h_{\text {eff }}$ (red dotted line).
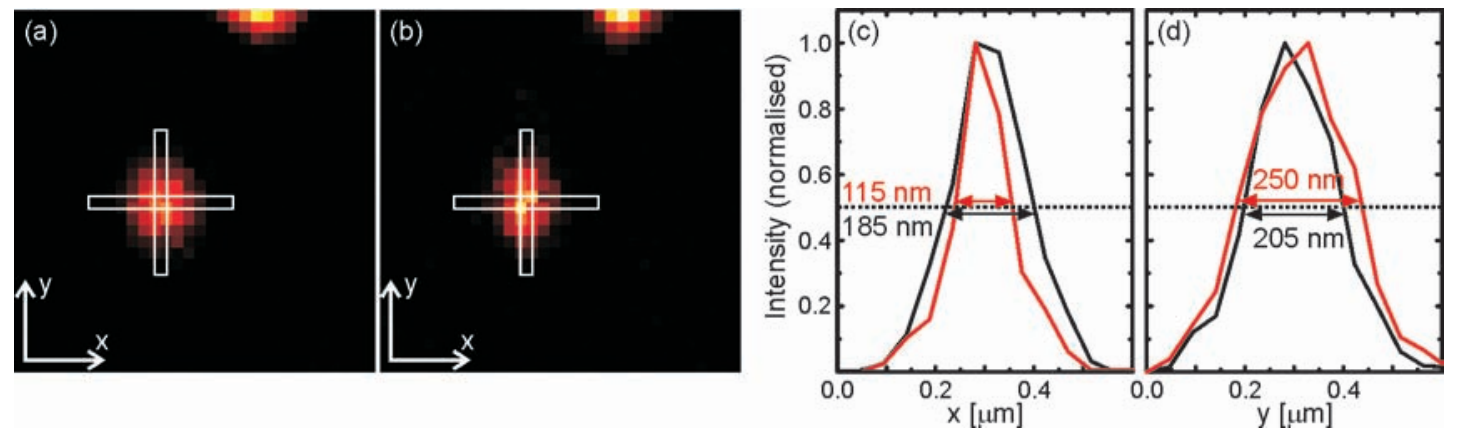

Figure 2.11: (a) Two dimensional confocal image of a single $30 \mathrm{~nm}$ bead. (b) RESOLFT-image of the same bead as in (a), resolution enhancement in $x$-direction. (c) Line profile of the average of the two central pixel rows ( $x$-direction), as indicated by the horizontal boxes in (a) and (b) for the confocal image (black line) and the RESOLFT-image (red line), respectively. (d) Line profile of the average of the two central pixel columns ( $y$-direction), as indicated by the vertical boxes in (a) and (b) for the confocal image (black line) and the RESOLFT-image (red line), respectively.

$h_{e f f} \ll h_{e x c}$. The expected $h_{\text {eff }}$ for the $I_{\text {off }}$ power setting of the measurement for figure 2.9 is calculated according to equation 2.1, using the fitted function $\eta$ of figure 2.10(a), the measured $h_{\text {off }}$ and a calculated $h_{\text {exc }}$. The results are shown in figure 2.10(b). The green line denotes the central profile in $x$-direction of $h_{e x c}$, the solid red line the one of the resulting $h_{\text {eff }}$. A convolution of our object of $30 \mathrm{~nm}$ in size, shown as black line, with $h_{\text {eff }}$ yields the red dotted line, that is the expected image of a single bead. The FWHM reads $85 \mathrm{~nm}$.

In order to investigate the feasibility of true two-dimensional images using this switchable marker, $30 \mathrm{~nm}$ nanoparticles were measured in confocal- and RESOLFT-mode. The scanning was performed with 32 steps for $1.5 \mu \mathrm{m}$ in both $x$ - and $y$-direction, resulting in a pixel side length of $\approx 50 \mathrm{~nm}$. Again, only resolution enhancement in $x$-direction was applied. The 
result is shown in figure 2.11(a) and (b), displaying the confocal and the RESOLFT image, respectively. Comparing the two, a narrowing of the bead image in $x$-direction can be observed in figure 2.11(b). This becomes even more obvious when looking at the line profiles in $x$-direction, which were taken by averaging the two central pixel rows as indicated by the horizontal white box and are plotted in figure 2.11(c). The RESOLFT image (red line) shows a FWHM of $115 \mathrm{~nm}$, whereas the confocal image shows a FWHM of $185 \mathrm{~nm}$. In the $y$-direction, however, no resolution increase can be observed, as can be seen in the profiles in 2.11(d), which show the average of the central two pixel columns in $y$-direction. The reason, why also the confocal image shows an aspect ratio of the $x$ - and $y$-profile $\neq 1$ is the linearly polarised excitation light, leading to an asymmetric $h_{e x c}$, which is enlarged in the direction of the polarisation. The intensities and lengths of the light pulses were $2.5 \mathrm{~ms}$ with an intensity of $380 \mathrm{~kW} / \mathrm{cm}^{2}$ for the activation light, $1 \mathrm{~ms}$ at $400 \mathrm{~W} / \mathrm{cm}^{2}$ for the switch-off light and $2 \mathrm{~ms}$ at $60 \mathrm{~W} / \mathrm{cm}^{2}$ for the excitation pulses.

Although an effect in resolution enhancement is clearly visible, the high resolution of the line-scans is not achievable in this two-dimensional measurement due to the limited number of switching cycles. Further research work concerning photostability of the DAE-unit is thus necessary.

\subsubsection{Application as Switchable Tracer}

A promising application for this kind of photoswitchable nanoparticles apart from high resolution imaging is to trace objects such as organelles or biomolecular agglomerations in a cellular environment. One can, for example, mark the beads from a certain region of interest by performing a switching reaction and then trace their motion within the cell. In order to do this, however, it is necessary to be able to detect the beads also in their non-fluorescent off-state. Therefore, $120 \mathrm{~nm}$ core-shell beads have been prepared, where a second dye, the non-switchable fluorophore Atto647N (Atto-Tech), is incorporated in the core of the beads and the Rh-AA-DAE is functionalised in the outer shell. A layer of $20 \mathrm{~nm}$ of pure silica separates the core from the shell, as shown in figure 2.12 (a). An electron microscope image of the actual beads can be seen in figure 2.12 (b). These beads can now be observed using two independent detection channels, one for the Rh-AA-DAE ('green') and one for Atto647N ('red'), which has its emission maximum at $670 \mathrm{~nm}$. Figures 2.12(c-f) show confocal images of these dual-stained beads. Figures 2.12(c) and (d) show the signal in the Rh-AADAE-channel before and after illumination with $\lambda_{o f f}=375 \mathrm{~nm}$. Typically, a contrast of 5:1 could be observed, depending on the irradiation times and intensities of the lasers. Figures 2.12(e) and (f) show the images in the Atto647N-channel. Here, the fluorescence signal remains unchanged, therefore this channel can be used for particle tracing while the signal in the green channel can be used for particle recognition. The size difference of the image of the beads between the green and the red channel stems from the size difference of the focal spot due to the different wavelengths of the excitation beams of $543 \mathrm{~nm}$ (green channel) and $671 \mathrm{~nm}$ (red channel). The on- and off-switching reactions of the particles can be performed at arbitrary locations by illumination with light of the corresponding wavelengths. 
(a)

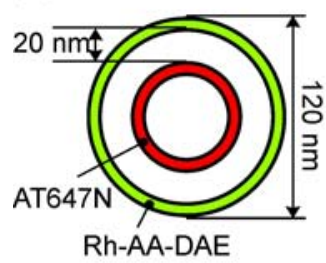

Rh-AA-DAE

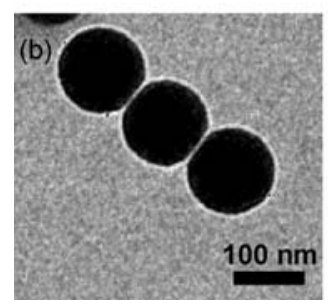

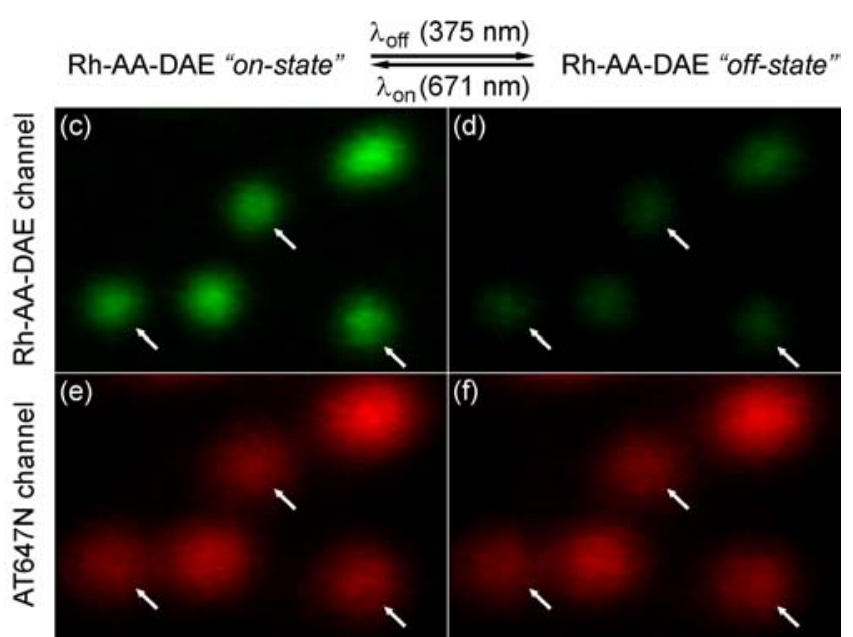

Figure 2.12: (a) Sketch of a dual-stained core-shell-bead. The inner core is doped with non-switchable Atto647N, the outer shell is doped with the Rh-AA-DAE, separated from the core with a $20 \mathrm{~nm}$ thick layer of pure silica. (b) Electron microscope image of the beads. (c) Signal of the Rh-AA-DAE channel with the beads in the on-state. (d) Signal of the Rh-AA-DAE channel with the beads in the off-state. (e) and (f) Signal of the Atto647N channel with the Rh-AA-DAE in the on- and off-state, respectively.

\subsubsection{Summary}

In this study the major problem of the photochromic system described in section 2.2 was tackled. For general use, the RET acceptor and the donor have to be covalently linked and equipped with a binding site. The applicability of a dyad, consisting of a photochromic diheteroarylethene as a RET acceptor and a covalently linked Rh101 as a donor, as a photoswitchable fluorescent compound was shown in this study. The fluorescent switch features a very good extinction ratio due to a high conversion to the dark state and highly efficient RET. Although this compound by itself is not hydrophilic enough to be directly used in aqueous environments, as for example in biological samples, it can be incorporated in silica nanoparticles, which are hydrophilic and furthermore fully biocompatible. Further, this approach leads to a signal increase.

These particles can for example be used as switchable tracers in biological experiments. Furthermore, the applicability of this compound for RESOLFT-type high resolution imaging was investigated. A clear resolution increase in the RESOLFT images is obvious. Measurements on $30 \mathrm{~nm}$ silica beads show a resolution of $75 \mathrm{~nm}$. Apart from its insolubility in water, the Rh-AA-DAE compound further suffers, however, from a switching fatigue of the photochromic DAE-unit which makes routine two-dimensional high resolution imaging on a level comparable to the performance of actual STED microscopes impossible at the present stage. Only 30 complete switching cycles can be performed in the given environment, before irreversible photodamage becomes significant, leading to less efficient fluorescence extinction and thus worse resolution. Several hundred to a thousand cycles would, however, be necessary. To widen this bottleneck, further research in this direction is required. 


\section{SMS-Microscopy}

SMS-microscopy uses a switching mechanism of fluorescent markers in order to generate a small subset of single, spatially separated fluorescing molecules in a densely stained sample. By successively localising their position, switching off the active subset, activating a new subset and repeating this process until a sufficient number of markers has been localised, the spatial distribution of the stained object is reconstructed [14, 15, 16]. Due to the fact that, given enough photons in the fluorescence signal of the molecule, the position of the dye can be estimated with an uncertainty much smaller than the diffraction limit of a conventional microscope and the pre-knowledge that the signal stems from just one dye molecule, the diffraction barrier can be broken.

In the framework of this thesis three major developments in SMS-microscopy have been achieved and will shortly be introduced in the following.

A SMS-microscope setup is based on a standard wide-field microscope. The lack of axial resolution represents a problem when imaging thick samples, due to disturbing fluorescence light from out-of-focus regions. In section 3.1, a solution to this problem is presented by optical sectioning with two-photon-activation of the marker dyes. A novel family of photoswitchable dyes is introduced which make this technique possible, allowing for the first time the imaging of thick samples using SMS-high resolution microscopy [25, 26].

Furthermore, a new method for simple and efficient multicolour SMS-microscopy which exploits the single-molecule approach of SMS-microscopy also for marker type discrimination was developed [27]. This method is presented in section 3.2 using different marker types of the novel dye family as well as photoswitchable proteins [28, 74].

Finally, SMS-microscopy based on ground state depletion is presented in section 3.3. This technique is an experimentally simple SMS-approach which makes possible the use of a wide range of standard dyes and fluorescent proteins for SMS-microscopy by exploiting their inherent electronic states for the switching mechanism even in live-cell environments [29].

\subsection{SMS-Microscopy With Optical Sectioning Using Two-Photon Activation}

The first demonstrations of SMS-microscopy were performed using thin samples, that is, objects which were thinner than the depth of field of the microscope [14, 15, 16, 17, 18]. Typical objects were slices of fixed cellular samples. Mostly, total internal reflection fluorescence (TIRF) is applied to reduce background from out-of-focus regions [14, 15, 16, 23, 24]. This, however, restricts imaging to the plane directly on the microscope coverslip and thereby abandons one of the most important advantages of light microscopy: noninvasive imaging 

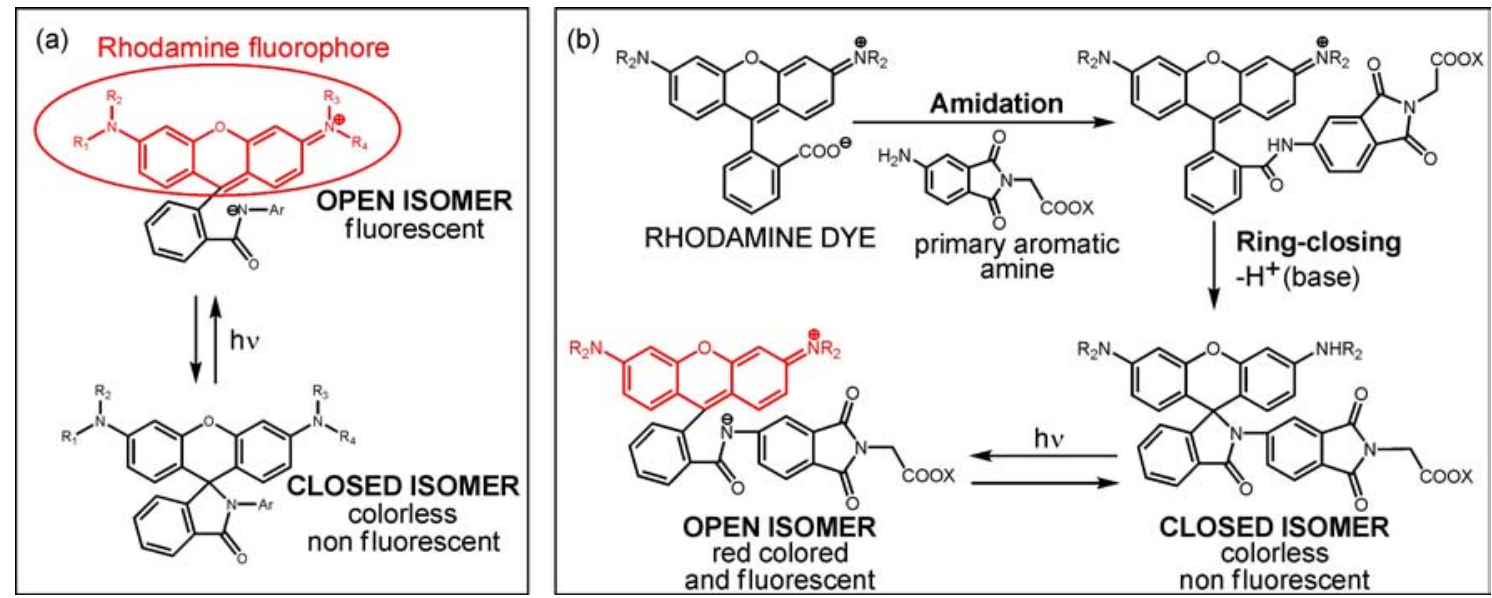

Figure 3.1: (a) General structure of a switchable rhodamine amide. The open, fluorescing form is shown on top with the actual Rhodamine fluorophore in red. The closed form is shown on the bottom. The opening reaction can be induced by light, the closing reaction is spontaneous with a lifetime between milliseconds and minutes, depending on the environment. (b) Chemical reaction scheme for the synthesis of the compound.

inside (whole) thick samples. Although it was demonstrated that TIRF-illumination is not mandatory, and background signal can be reduced with an optimised IRA recording scheme in Geisler et. al. [17], imaging was still restricted to thin samples in that work. In this thesis, a family of novel photoswitchable dyes is introduced, which makes it possible to image samples much thicker than the depth of field of the microscope by noninvasive optical sectioning using two-photon activation of the marker [25].

\subsubsection{The Novel Family of Photoswitchable Compounds}

The switchable photochromic compounds used for these experiments are based on rhodamine dyes. A photochromic reaction of rhodamine amides was already reported in the 1970s by Knauer and Gleiter [75]. Figure 3.1(a) shows a scheme of the reaction and figure 3.1.b) shows the steps involved in the synthesis of a representative dye. The open isomer (OF) on top of figure 3.1 (a) is fluorescent. Closing the bond leads to a rearrangement of the electron distribution of the rhodamine fluorophore such that it cannot absorb and fluoresce any more. This second isomer, the closed form (CF), is depicted on the bottom. It can be transferred to the OF by irradiation with near-UV light. The off-reaction is a thermally activated spontaneous process and cannot be controlled by light. These compounds were disregarded for a long time because of the low quantum efficiency of the switching process, the low number of switching cycles and the relatively short lifetime (milliseconds in case of the open isomer in polar solvents) [76]. Such properties are typically needed for applications in optical memories and switches [77], which were a major research goal at that time. The properties of this type of compound, however, make it an excellent candidate for SMS-microscopy, since it fulfills all of the criteria needed as stated in section 1.5.6.

- Rhodamine dyes are very photostable, therefore they give a very good signal, thereby 

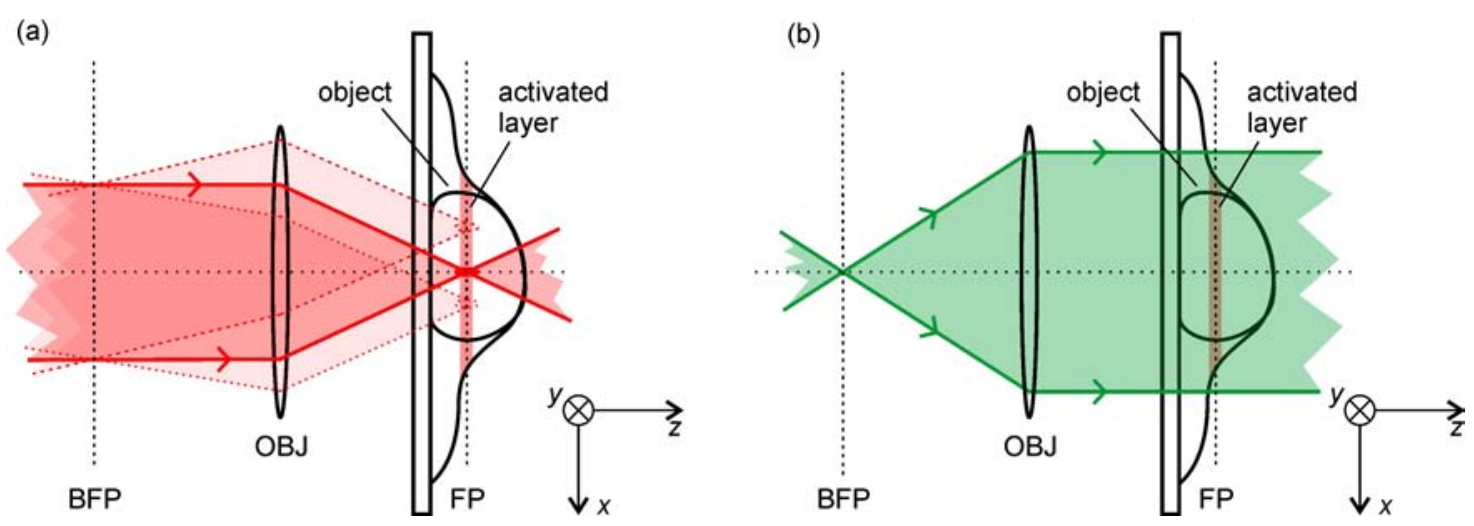

Figure 3.2: (a) Setup for 2-photon-activation (2PA). The collimated beam entering the objective (OBJ) is focussed in the focal plane (FP), where it has enough intensity to induce the 2PA process. The focus can be moved across the object by changing the angle of the incident light in the back focal plane (BFP) of the objective (beam paths shown with dashed and dotted lines). The activated region is thus restricted to the focal layer inside the object (labelled red area). (b) After activation, the dyes in the activated focal layer can be excited and imaged without affecting the non-activated molecules in the other regions of the object.

enabling very high resolution.

- The activation of the dyes can be easily controlled by light, the switch-off is performed by photobleaching.

- The ratio of initially activated dyes to the ones on the off-state is very low as will be seen later on.

- The contrast between the on- and off-states is virtually infinite.

The basic switching principle, shown in figure 3.1, works with a large number of dyes with different spectra and properties. This introduces a whole new family of dyes for SMS-type microscopy which are, as will be seen in the following, brighter than the fluorescent proteins used for the first SMS-experiments [14, 16, 17, 18], switchable and well suited for staining of biological samples.

\subsubsection{Optical Sectioning with Two-Photon Activation}

A major advantage of this dye family is the fact that the activation reaction can not only be induced by UV light but also by a two-photon process [78], where the simultaneous absorption of two photons in the red or near infrared range delivers the required reaction energy. The intensity $I_{2 P}$ of the two-photon activation (2PA) light has to be very high in order to obtain a sufficiently high probability for simultaneous absorption of two photons. Therefore, the 2PA-light is focussed into the sample as shown in figure 3.2 (a) and the focal spot is scanned over the whole field of view. The intensity outside of the focus is not high enough to induce the two-photon process. The reaction probability of the two-photon-process scales with the square of the intensity [78, 79]. Therefore, the activation is efficiently restricted to the focal layer of the 2PA-light. This layer can now be imaged without background light from 


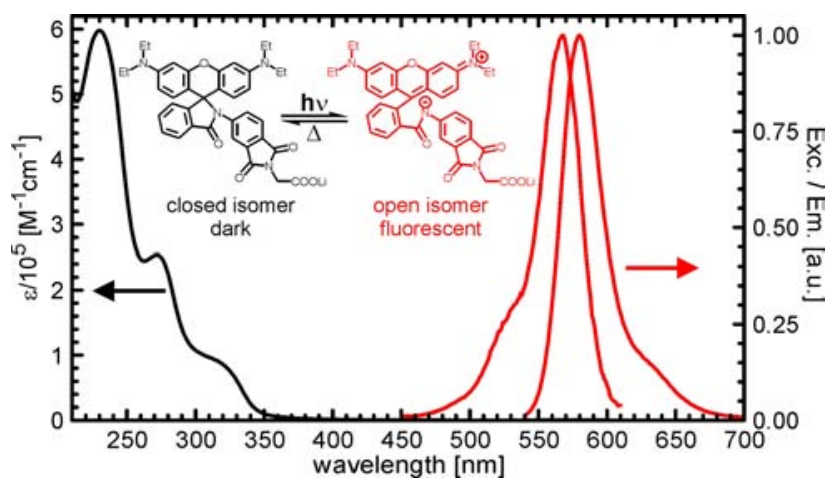

Figure 3.3: Absorption spectrum of the CF of SRA577 (black line, left axis) after illumination with $366 \mathrm{~nm}$ light and excitation and emission spectrum (red lines, right axis) of the OF. The inset shows the chemical structure of the actual SRA577.

out-of-focus regions causing a disturbance as shown in figure $3.2(\mathrm{~b})$, since those molecules remain in the off-state. Furthermore, as only the molecules in the focal layer are activated, only these are effected by photobleaching during the imaging process. The other unaffected parts of the sample can still be imaged layer by layer afterwards. The sample can thus be sectioned non-invasively by optical means.

This is analogous to two-photon scanning microscopy [79] but with the conceptual difference that in the latter case the molecules are excited layerwise, while in the case of 2PASMS-imaging the markers are activated layerwise. The excitation light is still sent in in a standard wide-field configuration, thus illuminating all layers of the sample homogeneously as shown in figure $3.2(\mathrm{~b})$.

\subsubsection{The Switchable Rhodamine Amide SRA577}

In order to demonstrate the applicability of the switchable rhodamines for SMS-microscopy in general and two-photon-sectioning in particular, a compound was chosen based on Rhodamine $\mathrm{B}(\mathrm{RhB})$ as the fluorophore, which is very photostable and water soluble, thereby facilitating the staining of biological samples. The quantum efficiency of the switching process in the near UV-range ( $360 \mathrm{~nm}$ to $380 \mathrm{~nm}$ ) was improved by using 4-Aminophthalimide as the aryl substituent to provide sufficient absorption in the near-UV. This is depicted in figure 3.1.b). However, since only sparse activation is needed, a very high quantum efficiency is not mandatory. The spectra of the compound, which is named SRA577 ('Switchable Rhodamine Amide', with emission maximum of $577 \mathrm{~nm}$ ) are shown in figure 3.3. The absorption spectrum of the CF is drawn with a black line, and the excitation and emission spectra of the OF after illumination with light of $366 \mathrm{~nm}$ with a red line. The inset shows the structure of the two isomers of SRA577, black for the CF, red for the OF. The OF reverts to the CF spontaneously with a kinetic constant which strongly depends on the solvent. Its lifetime is milliseconds in polar solvents but up to tens of minutes in the standard testing environment of the polymer poly(vinyl alcohol) (PVA) [76]. In the latter case, that is with long lifetimes, the 
switching-off of the dye during the measurement is performed by irreversible photobleaching. Thermal activation to the OF is marginal. Measurements in PVA after thermal relaxation to the equilibrium reveal that the fraction of detected spontaneously activated molecules is $<10^{-4}$. This number has been estimated by casting a dye-doped PVA-film onto a glass cover slip and counting the number of markers in the on-state above detection threshold in the field of view. The absolute number of markers in the field of view was determined from the known concentration of the dye with respect to the polymer, the measured thickness of the polymer film and its known density.

The two isomers show a huge contrast between the on-state and the off-state. Due to the rearrangement of the electron distribution of the Rhodamine, it cannot absorb any excitation light in the off-state, as can be seen by the absorption spectra in figure 3.3 , and is thus practically non-fluorescent. This also becomes obvious in the exemplary image of figure 1.9. where a large number of SRA577 markers in the off-state are present but do not produce any signal. In the on-state it is brightly fluorescing, yielding $\bar{N} \approx 2000$ photons using a threshold $T=300$ photons in the standard cell embedding medium Mowiol (see Appendix A.1). A major reason for the high fluorescence is the absence of a photoinduced switch-off reaction, which also eliminates undesired cross-talk between read-out and switch-off. SRA577 is thus much brighter than most of the fluorescent proteins which were used in other SMSrealisations, such as Dronpa, PS-CFP2 [23], PA-GFP [16] and rsFastLime [17, 18, 80]. For these proteins, only several hundred photons per event were measured on average. This leads to higher localisation accuracies in the case of SRA577.

Considering the properties of SRA577, it shows that it satisfies all the requirements for a SMS-dye as stated in section 1.5.6. According to equation 1.24, the theoretically achievable average resolution with this marker in the given environment and using the threshold stated above would be

$$
\Delta \tilde{r}=\frac{d_{\text {det }}}{\langle\sqrt{N / 2}\rangle} \approx 11 \mathrm{~nm},
$$

where $\langle\sqrt{N / 2}\rangle$ is the mean of the squareroots of half the photon numbers, $N$, of all events and $d_{\text {det }} \approx 270 \mathrm{~nm}$ the FWHM of the detection PSF.

\subsubsection{Experimental Setup}

The experimental setup is sketched in figure 3.4 . For the following experiments, pulsed 2PAlight was used in order to obtain a sufficiently high intensity for the 2PA process of SRA577. The light of $747 \mathrm{~nm}$ was provided by a Ti:Sa laser (Mira 900, Coherent Inc., Santa Clara, CA, USA) with a pulse frequency of $f_{747}=78 \mathrm{MHz}$ and transferred to the experimental setup by a low dispersion hollow core fibre (AIR-6-800, Crystal Fibre A/S, Birkerød, Denmark). The final pulse length was $t_{747}=5 \mathrm{ps}$. The beam was collimated and guided to the piezo-actuated scanning mirror SM2 (PSH 5/2 SG, Piezosystem Jena, Jena, Germany) which was used for the slow axis scanning of $30 \mathrm{~Hz}$. The plane of SM2 was then projected by lenses L5 and L4 onto the resonant fast-axis scanner SM1 (EOPC, Glendale, NY, USA), running at $14.6 \mathrm{kHz}$. Although not drawn to scale in figure 3.4, the planes of SM1 and SM2 were thus separated by the distance $2 \cdot f_{5}+2 \cdot f_{4}$, with $f_{i}$ being the focal length of the lens 


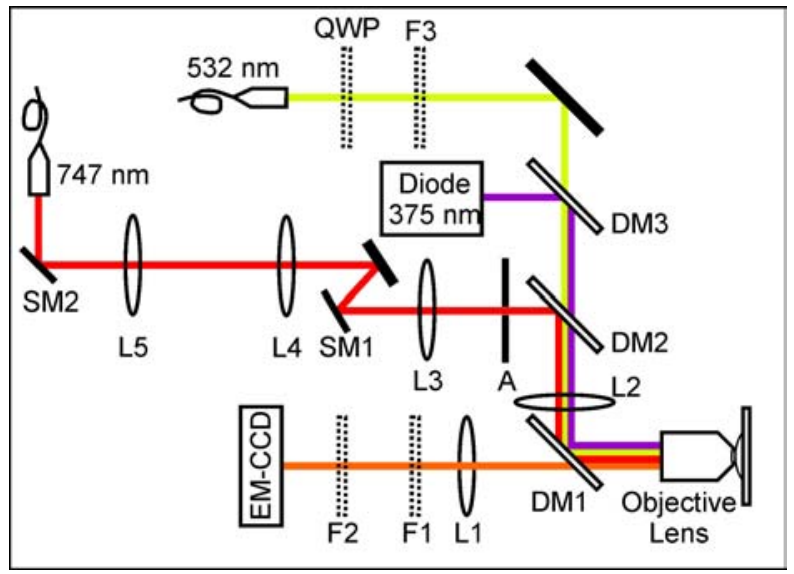

Figure 3.4: Sketch of experimental setup. L1-L5: Lenses with focal lengths $f_{1}=500 \mathrm{~mm}, f_{2,5}=300 \mathrm{~mm}$ and $f_{3,4}=80 \mathrm{~mm}$. DM1-3: Dichroic mirrors z532NIR/rpc (dual band reflection), z690sprpc (short pass) and z375rpc (long pass) respectively. F1-3: Filters NF01-532U-25, FF01-585/75-25 and z530/10x. SM1: $14.6 \mathrm{kHz}$ resonant galvo scanning mirror. SM2: Piezo actuated scanning mirror, running independently at $30 \mathrm{~Hz}$. QWP: $\lambda / 4$ wave-plate, A: Square aperture.

Li. The two lenses are positioned such that the scanning mirrors are in the focal planes of the respective lenses. Lenses L3 and L2 then project the plane of SM1 into the back focal plane of the objective (PL APO 100×, 0.7 - 1.4, Leica Microsystems, Wetzlar, Germany). The scanning beam therefore just changes its angle in that plane, where it is also collimated, leading to a position shift in the focal plane. A rectangular aperture A is placed in the focal plane of L3 which is also the back focal plane of L2, where the beam is focussed. The aperture ensures that only the quasi linear part of the sinusoidal scanning cycle corresponds to the field of view in order to provide homogeneous activation. Both scanners are running freely and are not synchronised. The complete field of view was thus illuminated with the 2PA-light at a frequency of $60 \mathrm{~Hz}$, considering that it is fully covered during both forwardand backward-movement of one oscillation of the slow mirror.

For comparison, cw-UV light of $375 \mathrm{~nm}$ was used for standard one-photon activation (1PA). It was provided by a laser diode (iPulse-375, Toptica) and focussed into the objective's back focal plane by lens L2, providing a quasi-wide-field illumination with a Gaussian intensity profile of FWHM of $16 \mu \mathrm{m}$ in the field of view of $12 \times 12 \mu \mathrm{m}^{2}$.

The cw-excitation light of $532 \mathrm{~nm}$ was provided by a fibre-coupled DPSS laser (Verdi V10, Coherent Inc.). It was circularly polarised by a $\lambda / 4$-waveplate (QWP), filtered with a cleanup filter and combined with the UV-light in the same beam path by the dichroic mirror DM3 and also focussed into the back focal plane of the objective by lens L2, providing an illumination with a Gaussian beam profile of FWHM of $12 \mu \mathrm{m}$. Both beams were combined with the 2PA-light by means of dichroic mirror DM1.

The fluorescence light was collected by the same objective, decoupled from the excitation and activation light by dichroic mirror DM1, filtered from remaining excitation and background light by a notch filter F1 and a bandpass filter F2, and projected onto the CCD of the camera with the help of tube lens L1 such that the focal plane of the microscope corresponds 
to the focal plane of the scanned 2PA beam.

Sample positioning was achieved by moving the object table using manual micrometre screws. For fine positioning in the axial direction, $z$, a piezo-actuated objective positioner was used (MIPOS 4 CAP, Piezosystem Jena).

\subsubsection{Experimental Results}

In order to demonstrate the applicability of SRA577 for SMS-microscopy, the microtubule network of fixed mammalian PtK2 cells was immunostained with SRA577 (for more detailed information on cell preparation and immunostaining, please refer to the appendix A. Microtubules have many known functions in the cell. They serve, for example, as a pathway for transportation inside the cell, along which motor proteins can carry diverse cellular material. They also play a role during cell division when the chromosomes are pulled apart. Furthermore, the network contributes to the stabilisation of the cell.

To prove the general applicability of SRA577 first wide-field photoactivation with the $375 \mathrm{~nm}$ light was applied. The fixed cells were mounted in standard Mowiol of $\mathrm{pH} 8$ and a thin part of a cell was placed in the field of view. For densely stained samples such as these, the first 1000 frames can be measured without activation light, using only spontaneous activation of the dye molecules. The activation light was then turned on and its dose increased as more and more dye molecules were photobleached, in order to keep the density of activated dye molecules at a reasonable level. Figure 3.5 (a) shows an SMS-image of the microtubule network obtained from 10240 frames with a camera frame rate of $100 \mathrm{~Hz}$, resulting in a total acquisition time of $108 \mathrm{~s}$. The average excitation intensity was $I_{\text {exc }}=18 \mathrm{~kW} / \mathrm{cm}^{2}$. The image is displayed in the histogram view. The image pixel size corresponds to $25 \mathrm{~nm}$ in the object plane. 245874 events were registered for this image.

For comparison, figure 3.5 (b) shows the corresponding conventional wide field image, obtained by summing up all frames after background removal. The 'pixelated' look of the conventional wide-field image here and also in all following conventional images in this thesis stems from the fact that the magnification of the microscope has been chosen such that the pixel size is optimal for the localisation algorithm (see section 1.5.3). It corresponds to $96 \mathrm{~nm}$ in the object space. The detection PSF has a corresponding FWHM of $\approx 270 \mathrm{~nm}$. The sampling of the image is therefore below the Nyquist-Shannon criterion requiring the sampling to be at least twice as fine as the resolution [23, 81]. A smaller camera pixel-size would yield a smoother image but not more information. The same effect would be obtained if the image at hand was to be interpolated, which would also not yield more spatial resolution. It has therefore been chosen to display the raw data instead.

In order to demonstrate two-photon activation, the same sample was imaged again, now using 2PA with the $747 \mathrm{~nm}$ light with an average intensity $I_{2 P A}=35 \mathrm{MW} / \mathrm{cm}^{2}$. Figure 3.5 (c) shows the resulting image, obtained from 20480 frames at the same frame rate and $I_{\text {exc }}$ and displayed with a pixel size of $25 \mathrm{~nm}$. 364791 events were registered. The image is virtually identical to that obtained with 1 PA. In both cases, the lower photon threshold $T$ was set to $\approx 300$ in the localisation routine and the resulting average photon number was $\bar{N}=2100$. The histogram of the photon number distribution for the 2PA case is shown in figure 1.11 . The image acquisition time was technically limited by the speed of the scan- 

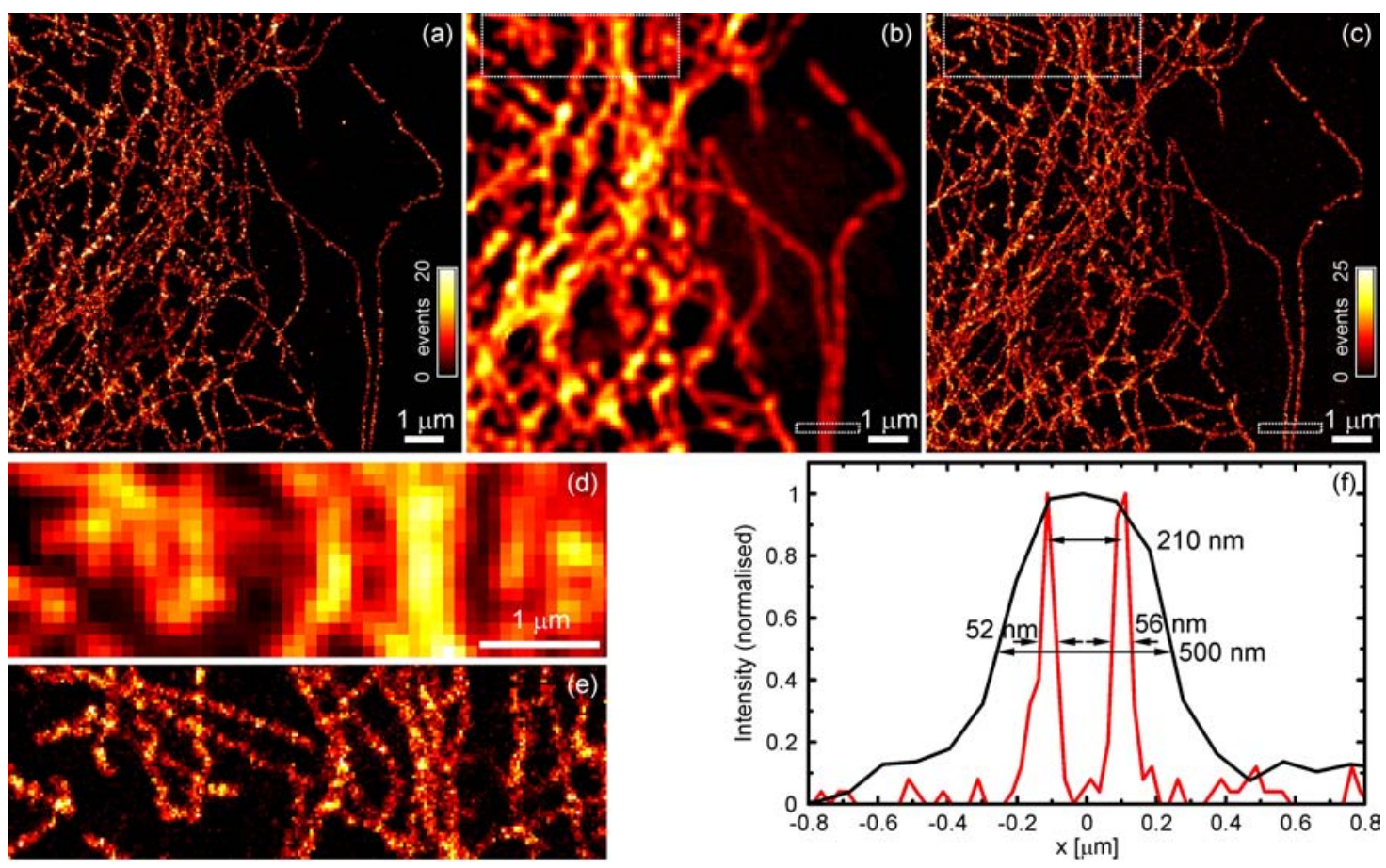

Figure 3.5: (a) SMS image in histogram-view of tubular network of an intact PtK2 cell stained with SRA577 using 1PA. 10240 frames were used. (b) shows the wide-field counterpart obtained by adding up all frames after background removal. The difference in resolution is obvious. (c) shows the same area of the cell as in (a) only using 2PA of $747 \mathrm{~nm}$ for activation. The image is virtually identical to the one in (a), showing that 2PA is applicable in a standard cellular environment. (d) and (e) depict the areas denoted in (b) and (c), respectively, by the large dotted rectangles enlarged, to make the differences in resolution more obvious. (f) is a plot of a few averaged horizontal ( $x$-direction) lines as shown by the small dotted boxes in (b) and (c) in black and red, respectively. The two parallel fibres cannot be resolved in the classical image but can be well resolved in the SMS-image. The colourbars in (a) and (c) denote the number of registered events per pixel.

ning system $(30 \mathrm{~Hz})$, leading to an activation frequency of $60 \mathrm{~Hz}$, but can in principle be accelerated. Figures 3.5 (d) and (e) show enlarged images of the region marked by the large white box in figure 3.5 (b) and (c), respectively, where the difference in resolution becomes obvious. Figure 3.5 (f) displays a profile of several averaged pixel rows, as denoted by the small white boxes in figure 3.5 (b) and (c), showing two parallel fibres. They can be much better resolved in the SMS-image as compared to the conventional wide-field image. The width of each fibre is determined to be $55-70 \mathrm{~nm}$, which corresponds well to the expected size of the microtubules of $25 \mathrm{~nm}$ plus primary and secondary antibody of the labelling on either side of the fibril (please see appendix A.2 for information on immunostaining). The cells were fixed and embedded in Mowiol of $\mathrm{pH} 8$.

In a second experiment, the possibility of optical sectioning using 2PA was demonstrated. For this, $5 \mu \mathrm{m}$, amino-modified, silica beads were surface-stained with SRA577 and imaged using 2PA at different $z$-positions at distances of $d=330 \mathrm{~nm}$ apart. The beads could therefore be imaged slice by slice, where the image of each slice is a projection of the 

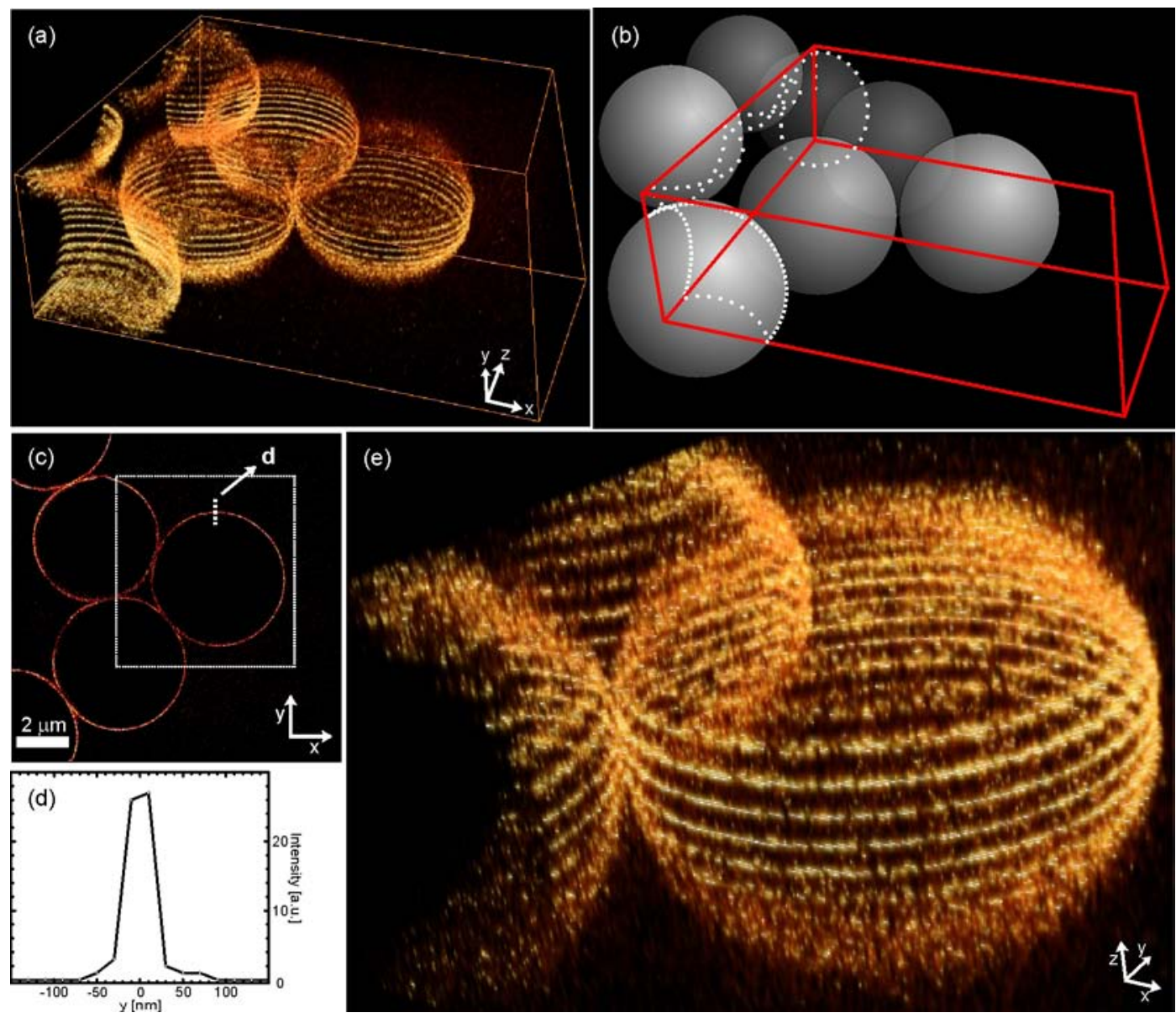

Figure 3.6: Application of optical sectioning. (a) 3D-reconstruction of 17 slices with a distance of $d=$ $330 \mathrm{~nm}$ taken by 2PA-optical sectioning. Note that the beads are in two layers. (b) Scheme of the complete beads of (a) to show their positions. (c) Slice closest to the equator of the beads. (d) Line profile at the spot denoted in (c) with a dotted line, the FWHM is $40 \mathrm{~nm}$. (e) Enlarged area of the beads, as shown with the dotted white square in (c).

$\approx 650 \mathrm{~nm}$ thick layer activated by the focussed $2 \mathrm{PA}$ light, whose average intensity was up to $I_{2 P A}=50 \mathrm{MW} / \mathrm{cm}^{2}$ in the focus. Figure 3.6 (a) shows the reconstructed three-dimensional image from 17 slices taken from seven beads. Five of the beads are ordered in one layer on the coverslip. Additionally, the lower half of two beads located in a second layer in the holes between the beads of the first layer can be seen on the left side. Figure 3.6(b) shows the actual arrangement of the entire beads in a scheme. The bead parts imaged in figure 3.6(a) are marked by white dotted lines.

Since each slice represents a two-dimensional projection of the $\approx 650 \mathrm{~nm}$ thick activated layer, the beads' rim appears wider for slices further away from the equator due to the curvature of the beads. For a slice exactly at the equator, $10 \mathrm{~nm}$ wide lines are expected. A slice at a distance $d / 2=165 \mathrm{~nm}$ from the equator would already show a rim of $24 \mathrm{~nm}$. The exact $z$-position of the equatorial plane was unknown in the experiment. Figure 3.6(c) displays the slice with the thinnest rim, that is the one closest to the equator of the lower beads. Its 


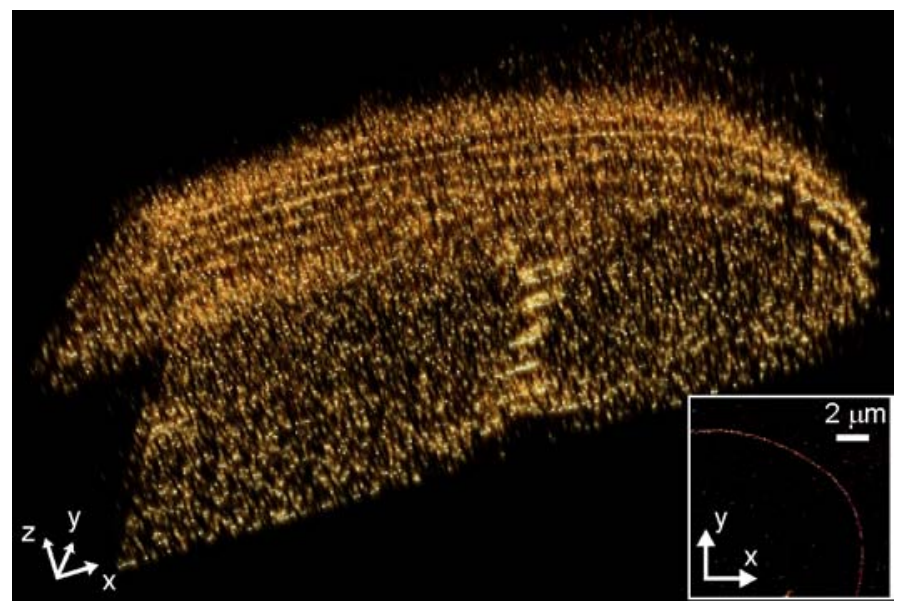

Figure 3.7: 3D-reconstruction of lamina skeleton of intact cell nucleus. The respective layers were taken using 2PA-optical sectioning. The inset shows the plane closest to the centre of the nucleus.

distance to the equator is therefore $\leq d / 2$. A line profile of the rim at the place of the dotted white line is plotted in figure 3.6(d). The FWHM is $40 \mathrm{~nm}$. Given the uncertainty of the expected width of the rim, the lateral resolution for each slice can therefore be estimated to be $\approx 20-30 \mathrm{~nm}$.

The area marked by the dotted square in figure 3.6(c) is shown in the 3D-reconstruction in a closer view in figure 3.6.(e).

To demonstrate the feasibility of applying 2PA-SMS-microscopy with axial sectioning in biological samples, labelled lamin proteins in the nucleus of human U373MG cells were imaged. Lamin serves as a skeleton for the nucleus, preserving its shape and stability. The cells were again mounted in Mowiol at $\mathrm{pH}$ 8. Figure 3.7 shows ten slices with a distance of $500 \mathrm{~nm}$ in $z$-direction of a section of the nucleus. The lamina is clearly observed. Note the tube-like connection of the top and bottom surface in the foreground, which is characteristic for this structure. Again, the lateral resolution in the equatorial plane is excellent as can be seen in the inset of figure 3.7, showing the viability of SMS-imaging even in thick biological samples $(>6 \mu \mathrm{m})$ without the need of further treatment, such as (cryo-)sectioning.

\subsubsection{Two-Photon Activation with cw-lasers}

The experiments described above were performed with pulsed laser light from a Ti:Sa laser in order to obtain the requisite high intensities for activating SRA577. This system is rather complex and expensive. In principle, however, it is also possible to achieve 2PA using focussed cw-laser light, similar to the case of two-photon excitation microscopy [82], so that less costly light sources become applicable. However, in order to reach the same intensity as the average intensity of a laser pulse in the pulsed case, a factor of $t_{747} \cdot f_{747} \approx 2500$ of higher average intensity is in principle needed in the cw-case, where $t_{747}=5 \mathrm{ps}$ is the pulse 

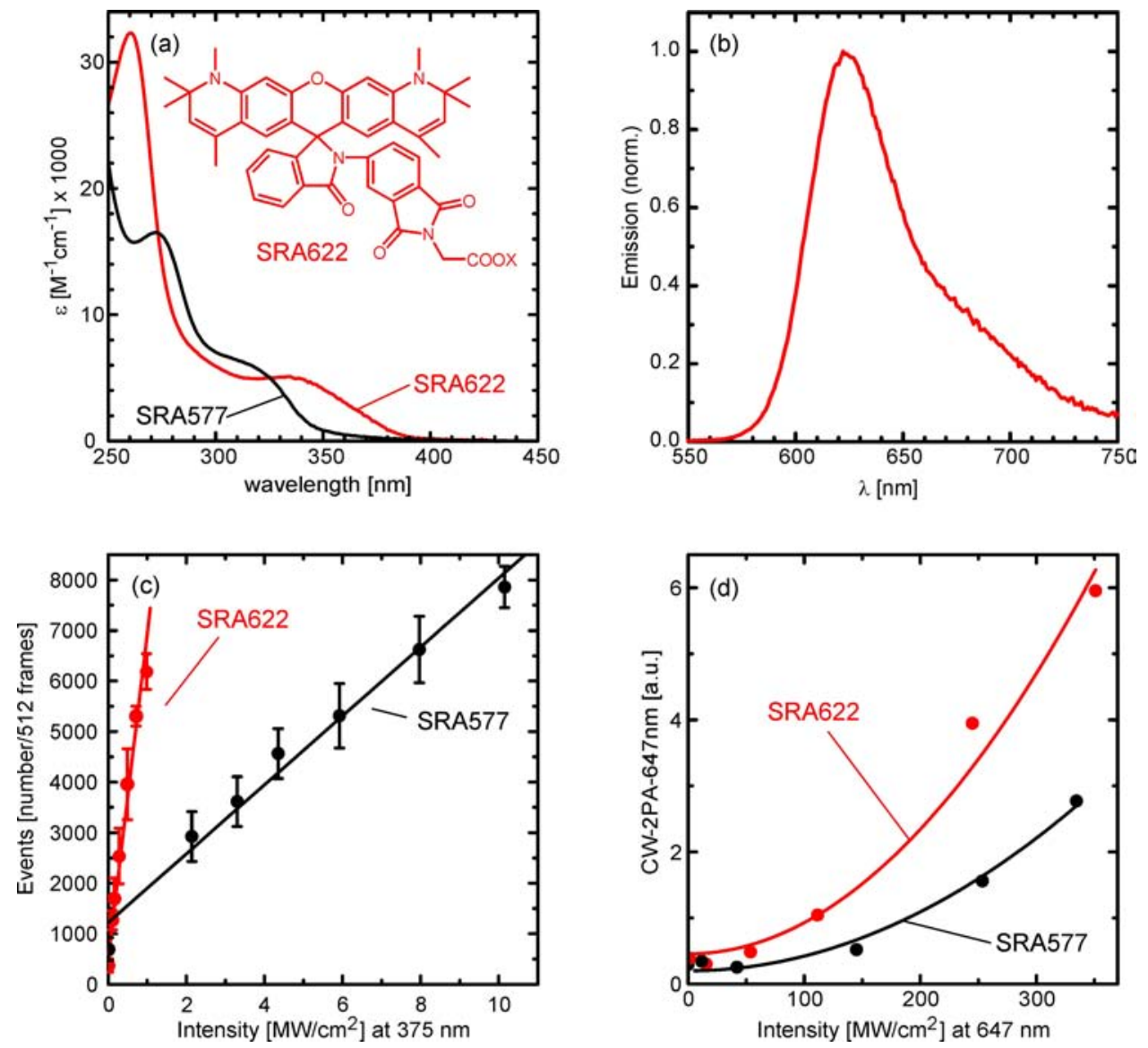

Figure 3.8: (a) Absorption spectra of the closed form (off-state) of SRA577 (black line) and SRA622 (red line). The Inset shows the chemical structure of SRA622. (b) Emission spectrum of the open form (on-state) of SRA622. (c) Activation of SRA577 (black circles) and SRA622 (red circles) upon irradiation with $375 \mathrm{~nm}$ light. The straight lines are linear fits. (d) Activation of SRA577 (black circles) and SRA622 (red circles) upon irradiation with $647 \mathrm{~nm}$ light. The solid lines are parabolic fits. The quadratic behaviour of the activation shows the existence of a two-photon absorption process. The activation in (c) and (d) is measured in number of recorded events per 512 frames.

length and $f_{747}=78 \mathrm{MHz}$ the pulse frequency of the $747 \mathrm{~nm}$ light used for the experiments described in section 3.1.5. In order to examine the possibility of 2PA, we applied a Krypton ion laser (Innova Sabre, Coherent) delivering cw-light of $647 \mathrm{~nm}$ and $670 \mathrm{~nm}$, but any laser, such as compact solid state lasers, can in principle be used. For these shorter wavelengths, the 2PA-cross section of SRA577 is expected to be larger in analogy to the 1PA-cross section depicted in figure 3.3 and figure 3.8 (a) with black lines. This partly compensates for the lower focal intensities reached in the case of $\mathrm{cw}$-excitation. The available focal intensity was $400 \mathrm{MW} / \mathrm{cm}^{2}$, which is only a factor of eight higher than the maximum available average power of the pulsed case. However, no activation could be observed for SRA577 in the case of $670 \mathrm{~nm}$ light and the effect using $647 \mathrm{~nm}$ light was insufficient for imaging purposes. Therefore, a new marker has been designed and synthesised, based on Rhodamine 590 with a more red-shifted absorption and emission of the OF. It is called SRA622 according to 
its emission maximum at $622 \mathrm{~nm}$. The emission spectrum of the OF is shown in 3.8(b). The compound is sketched as an inset in figure 3.8(a). The extra double bond and the rigid framework of SRA622 result in a considerably red shifted absorption of the CF as well, as can be seen in 3.8 (a) with the red line, in comparison to the one of SRA577, plotted with the black line. This red-shift in the absorption spectrum resulted in a ninefold higher activation efficiency with $375 \mathrm{~nm}$ light in PVA (PVA dissolved in buffer at $\mathrm{pH}$ 8.5, doped with dye and spin coated on cover glass), as compared to SRA577. Figure 3.8.(c) shows the activation versus irradiation intensity of the $375 \mathrm{~nm}$ light with the black circles for SRA577 and red circles for SRA622. An increase in activation efficiency in PVA can also be observed for two-photon-activation, as shown in figure $3.8(\mathrm{~d})$. Note that the number of activated dye molecules per frame increases linearly upon irradiation with $375 \mathrm{~nm}$ light in figure 3.8(c), since it is a one-photon absorption process. In case of irradiation with the $647 \mathrm{~nm}$ light, the activation follows a parabola (fit in figure 3.8(d) drawn with lines of the respective colours), showing that the activation reaction is indeed proportional to the square of the intensity, which is characteristic for a two-photon absorption process.

SRA622 shows a similar behaviour as SRA577 with respect to thermal recovery, high brightness of the emitting isomer, contrast between the two isomers and a low fraction of spontaneously activated markers.

Apart from the different laser source, only minor changes had to be applied to the setup. The most significant is that, due to the different activation wavelength, the activation beam could not be coupled into the objective by DM1. Therefore, DM2 was removed and an additional dichroic mirror was introduced between DM1 and the objective (z635 DCSPXR, AHF, Tübingen, Germany) which now couples in the 2PA light. Also an additional lens with $f=300 \mathrm{~mm}$ was now necessary for projection of the scanning mirror planes into the back aperture of the objective. DM1 was exchanged by a new dichroic (z532rpc, AHF).

To demonstrate the applicability of SRA622 in SMS-microscopy, small silica beads were prepared, which feature a core doped with SRA622 and a pure silica shell on the outside. The beads were dispersed in ethanol and samples were prepared by distributing a drop of the dispersion onto a standard microscopy cover slip and letting the ethanol evaporate. Figure 3.9 (a) shows an SMS-image in Gaussian view of such a sample, obtained from 45000 frames, recorded with a rate of $100 \mathrm{~Hz}$ (7.5 min total recording time), using 1PA with $375 \mathrm{~nm}$ light. The excitation intensity was $18 \mathrm{~kW} / \mathrm{cm}^{2}$. It can be seen that the beads are inhomogeneous in size and shape. This impression is affirmed by an electron microscope image of a similar sample in figure 3.9 (b). The average centre-centre distance of $118 \mathrm{~nm}$ of the beads obtained from the electron microscope images corresponds well to the distance of $120 \mathrm{~nm}$ found for the SMS-image. The wide-field counterpart of figure 3.9 (a) can be seen in figure 3.9.(c), it was again obtained by summing up all the single molecule signal after background removal. No single beads can be discerned in this image. The average photon number was $\bar{N}=1600$ after applying a threshold $T=200$ photons. These experiments show that SRA622 is similarly suited for SMS microscopy as SRA577.

In order to demonstrate the 2PA optical sectioning capabilities also with this marker, 


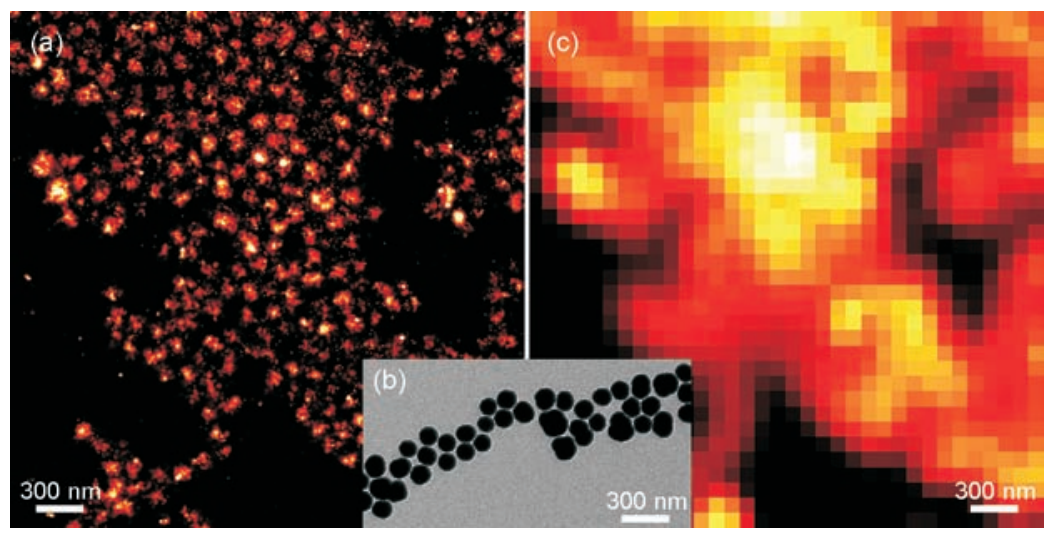

Figure 3.9: (a) SMS-microscopy picture of core-shell silica beads doped with SRA622 in Gaussian view. Only the doped cores are visible.(b) Electron microscope image of the whole beads. (c) The corresponding classical wide-field image to (a), reconstructed by adding all frames.

$5 \mu \mathrm{m}$ silica beads were surface-stained with SRA622 and layerwise imaged using 2PA light of $647 \mathrm{~nm}$ with a focal intensity of $350 \mathrm{MW}$. The sample was embedded in standard immersion oil. The scanning speed was the same as described in section 3.1.5, the frame rate was $100 \mathrm{~Hz}$ and the excitation intensity was $18 \mathrm{~kW} / \mathrm{cm}^{2}$. The sectioning capability is as good as for pulsed 2PA, detailed images were obtained from each slice without depleting the markers in the rest of the sample. The three-dimensional reconstruction from 16 slices of a single bead with a distance of $300 \mathrm{~nm}$ is shown in figure 3.10(a). Again, the lateral resolution is excellent, yielding $\approx 40 \mathrm{~nm}$ for the FWHM of a line profile close to the equatorial layer. The image of the layer is shown in figure 3.9 (b), the line profile in figure 3.9. (c).

\subsubsection{Summary}

This section introduced SMS-microscopy using two examples of a family of novel photoactivatable markers based on Rhodamine dyes. For the first time, fast imaging with independently running acquisition was combined with bright organic dyes in a far field-illumination setup, which allows for the imaging of thick samples. The new markers fulfil all requirements for SMS microscopy, that is easily controllable activation rate by light, a high number of photons, a perfect contrast between on- and off-state and a very low fraction of spontaneously activated markers. The switchable rhodamine amides consist only of one entity which can be easily functionalised so that staining of technical and biological samples is easily achieved with standard staining procedures. Their brightness surpasses the one of most switchable proteins used for SMS so far, such as Dronpa, PS-CFP2 [23], PA-GFP [16] and rsFastLime $[17,18,80]$ leading to higher localisation accuracies. The major advantage, however, is the ability to activate the markers using a two-photon activation process. Due to the required high intensity and the quadratic dependence of the activation process on the intensity, the activation light has to be focussed into the sample and the activation is efficiently restricted to the focal plane. Scanning the focus enables a homogenous activation of the whole focal plane, allowing one to image that part of the sample without affecting any other regions. 

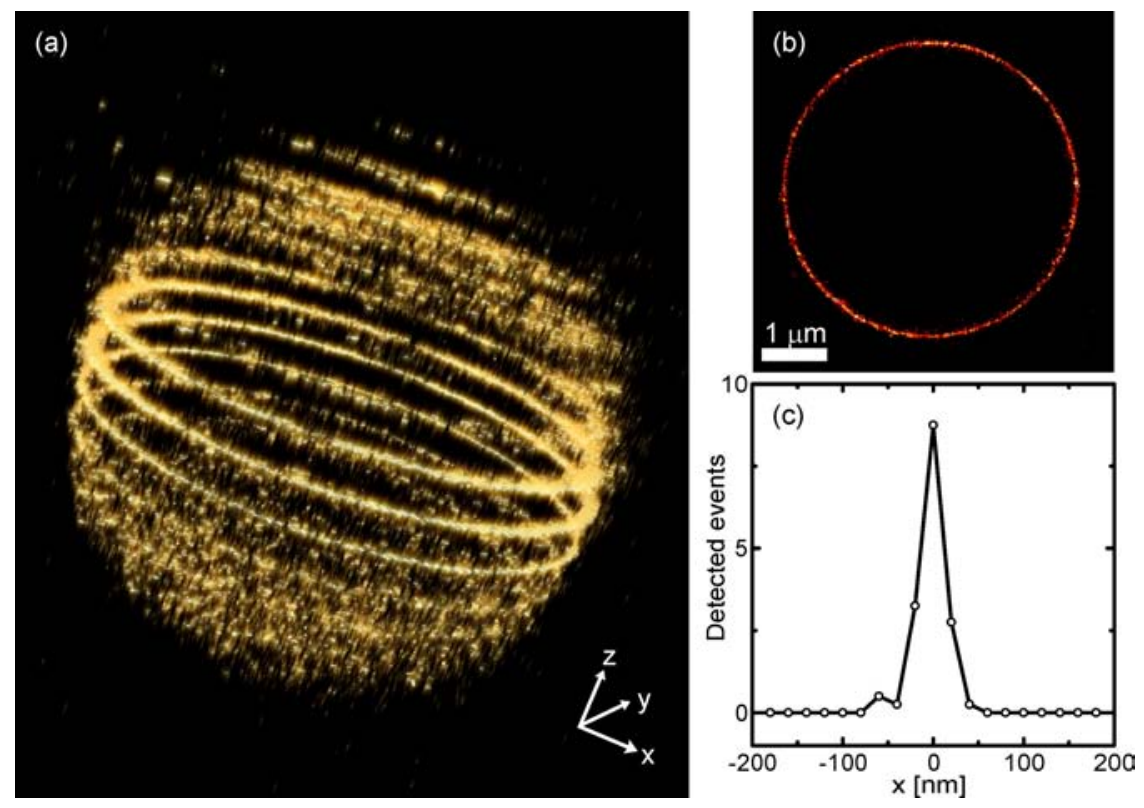

Figure 3.10: Optical sectioning of silica bead using cw-2PA. (a) 3D-reconstruction from 16 slices of a SRA622-surface-stained $5 \mu \mathrm{m}$ bead, which were taken using cw-2PA. (b) SMS-image from the equatorial slice in histogram-view. The pixel size is $20 \mathrm{~nm}$. The image consists of 6765 events taken in 10240 frames. (c) Line profile from (b), showing the high resolution within one plane.

Thick beads and cellular samples were thus imaged using SMS-microscopy for the first time by layerwise activation and imaging. The previously mandatory mechanical slicing of thick objects before imaging can thus be omitted, returning one of the most important features of light microscopy to SMS-imaging, that is the noninvasive imaging of thick samples. Each slice represents the two-dimensional projection of the activated layer, which has a thickness of $\approx 650 \mathrm{~nm}$, corresponding to the size of the activation PSF in the axial $z$-direction.

It was shown that the two-photon activation process is also applicable using $\mathrm{cw}$-light, making it possible to use cost efficient and compact easy-to-use diode- or solid-state-lasers for the activation process. For future use, even subdiffration localisation in the $z$-direction within one activated slice can be introduced, using artificial $z$-dependent asymmetries such as defocus or astigmatism [52, 83, 84, 85, 86, 87]. This enables full three-dimensional high-resolution SMS-microscopy in thick samples.

\subsection{Multicolour SMS-Imaging by Single Molecule Spectroscopy}

Labelling of different structures or molecular species with discernable fluorescent marker types enables one to elucidate the spatial and temporal correlation of these species. Apart from the noninvasive imaging of thick (living) cellular samples, this has made light microscopy an indispensable tool in biological research, despite the lack of high resolution. 
Therefore, implementing dual- or even multicolour imaging in the superresolution imaging concepts opens up an even wider range of possibilities for biological research. First in STEDmicroscopy as the RESOLFT-representative [88], then subsequently in SMS-type imaging, the possibility of multicolour superresolution light microscopy has been shown [23, 24, 80]. In classical fluorescence light microscopy, the signal stems from a large number of dye molecules simultaneously. Thus, the resulting emission spectrum is composed of the photons from all markers involved. For an efficient unmixing of the marker signals, and thus the discrimination of their types, spectrally well separated dyes are used, which are typically excited and/or detected with separate light sources and detection channels. This also applies for RESOLFT-microscopy. For SMS-imaging, the activation presents another alternative to discriminate the different markers. If the activation efficiency of different marker types is wavelength-dependent, specific light sources can be used to activate the different marker types individually [24]. So far, all superresolution multicolour approaches have followed this classical path which requires multiple activation, excitation and/or detection channels for multiple markers and thus increases the requirements of the spectroscopic properties of the dyes and on the light sources and hence the complexity of the experiment. Further, the sequential imaging of the different markers, which can often not be avoided due to the overlap of the emission spectrum of one dye and the activation or excitation light for another, compromises the accuracy of the measurement due to sample movements or drifts and results in slower image acquisition.

In this section, a method is presented which enables simultaneous multicolour SMS-imaging using only one excitation and activation channel and two detection channels by making use of the emission properties of the single molecules [27].

In the following, the theoretical background of the underlying idea will be explained and compared to the experiment. Then, the markers used will be introduced and the experimental setup and data processing will be explained. Finally, results of actual measurements will be presented and a summary of the results will be given.

\subsubsection{Colour Separation Principle}

In conventional multi-colour microscopy, each individual photon has to be regarded as one measured event. In order to determine from which marker type it came, only the detection channel where it ended up can be used. Therefore, in order to ensure with a high degree of certainty that the photon ends up in the right channel and is thus attributed to the right marker type, the spectra and channels have to be well separated to provide efficient unmixing of the markers' signal. Since SMS-microscopy relies on imaging single molecules, the unmixing of the markers is already inherently given by the method, because each recorded singlemolecule photon burst, consisting of $N$ photons, can only stem from one single marker. Therefore, a large number of photons can be associated with one molecule, opening up the possibility of determining the type of the marker by its spectroscopic properties with statistical relevance. This is possible even for small spectroscopic differences between the markers. In order to determine the marker type, any sufficiently prominent dye-specific property can be used. In the present case, the brightness and emission spectrum will be exploited, but in principle properties such as lifetime or anisotropy can also be utilised [89, 90, 91, 92, 93]. 


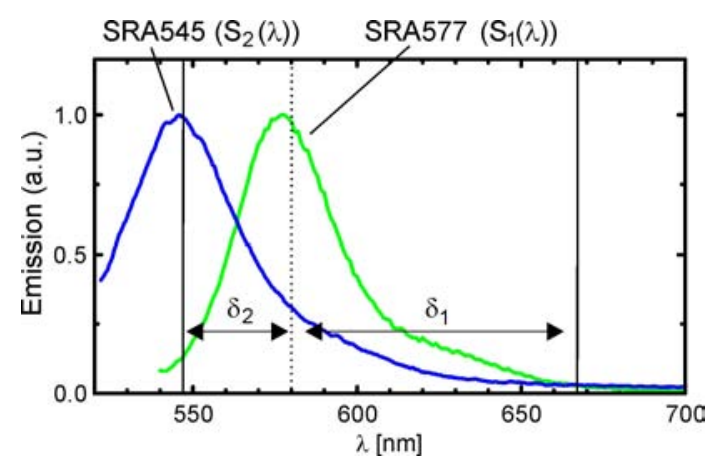

Figure 3.11: Emission spectra of the photoactivatable markers SRA545 (blue) and SRA577 (green). The solid vertical lines at $547.5 \mathrm{~nm}$ and $667.5 \mathrm{~nm}$ denote the total detection window, the dotted line at $580 \mathrm{~nm}$ represents the border between the two detection channels.

Differentiation of dyes based on their brightness and emission spectrum can be efficiently achieved by dividing the fluorescence signal from each single-molecule burst into two neighbouring, yet spectrally separated detection channels and using the number of photons detected in the respective channels as an identification criterion for the marker type. The general theoretical background will be discussed in the following in analogy to the work by Middendorff [52] but with focus on the experimentally relevant case of only two detection channels.

Imagine a number $L$ of markers with emission spectra $S_{l}(\lambda), l=1 . . L$, emitting the mean photon numbers $\bar{n}_{l}$, and $C$ detection channels which have the adjacent transmission windows $\delta_{c}, c=1 . . C$. Figure 3.11 shows the example for $C=2$ and $L=2$ with the spectra $S_{1}(\lambda)$ and $S_{2}(\lambda)$ of the dyes SRA577 (green line) and SRA545 (blue line), respectively. The two solid vertical lines at $547.5 \mathrm{~nm}$ and $667.5 \mathrm{~nm}$ represent our total detection window and the dotted vertical line at $580 \mathrm{~nm}$ represents the border between $\delta_{1}$ and $\delta_{2}$. Note that $\delta_{1}$ is the window corresponding to the longer wavelengths. The mean signal in the channels $\bar{c}_{j}, j=1 . . C$ for the normalised case of one marker per type is

$$
\overline{\mathbf{c}}=\mathbf{K} \overline{\mathbf{n}},
$$

where $\overline{\mathbf{c}}=\left(\bar{c}_{1}, . ., \bar{c}_{C}\right)$ and $\overline{\mathbf{n}}=\left(\bar{n}_{1}, . ., \bar{n}_{L}\right)$ are column vectors and the sorting matrix $\mathbf{K}$ of dimension $C \times L$ has the elements

$$
K_{c l}=\frac{\int_{\delta_{c}} S_{l}(\lambda) d \lambda}{\int_{\delta} S_{l}(\lambda) d \lambda}
$$

Here, $\delta$ represents the total detection window. $K_{c l}$ is the probability that a photon from molecule of type $l$ will end up in channel $c$. For the configuration shown in figure 3.11, we get the exemplary sorting matrix

$$
\mathbf{K}=\left(\begin{array}{ll}
0.54 & 0.25 \\
0.46 & 0.75
\end{array}\right)
$$


The columns of $\mathbf{K}$ correspond to the marker types in the order SRA577, SRA545 and the rows are the corresponding detection windows in the order $\delta_{1}, \delta_{2}$, that is of decreasing wavelengths.

In the conventional case of multicolour microscopy, where each detection channel is associated with one dye, it is necessary to minimise the off-diagonal elements of $\mathbf{K}$ in order to minimise signal crosstalk to that one is still able to obtain quantitative information about the densities of the marked species. Practically, this is done by separating the spectra and detection windows as much as possible. The sorting matrix $\mathbf{K}$ in equation 3.4 is thus very unfavourable and linear unmixing would have to be applied, resulting in increased noise (as discussed later).

In the case of SMS-microscopy, the columns of $\mathbf{K}$ only give the expected distribution of the photons of one marker type from one burst into the channels which can then be used as a maximum likelihood estimator to determine which type it was most likely. Therefore, it does not matter if the photons of one marker are distributed into both channels, as long as the distribution is characteristic and differs sufficiently from the ones of the other markers. From the theoretical viewpoint, the assignment of a marker to a given signal is straightforward and based on the photon statistics [52]. Consider a single molecule event yielding the photon distribution $\mathbf{c}$. The probability $P(\mathbf{c} \mid l)$ of that channel signal configuration given that it stems from molecule $l$ reads

$$
P(\mathbf{c} \mid l)=\frac{N !}{c_{1} ! c_{2} ! \ldots c_{C} !} K_{1 l}^{c_{1}} \ldots K_{C l}^{c_{C}}
$$

where the total measured photon number $N$ is given by

$$
N=\sum_{j=1}^{C} c_{j} .
$$

In the experimentally simplest case of two detection channels equation 3.5 results in a binomial distribution:

$$
P(\mathbf{c} \mid l)=\frac{N !}{c_{1} ! c_{2} !} K_{1 l}^{c_{1}} K_{2 l}^{c_{2}}=\frac{N !}{c_{1} ! c_{2} !} K_{1 l}^{c_{1}}\left(1-K_{1 l}\right)^{c_{2}} .
$$

For a measured distribution $\mathbf{c}=\left(c_{1}, c_{2}\right)$, one can now simply associate that molecule type $l^{\prime}$ to the measurement where $P\left(\mathbf{c} \mid l^{\prime}\right)=\max _{l}\{P(\mathbf{c} \mid l), l=1 . . L\}$, that is

$$
l^{\prime}=\arg \max _{l}\{P(\mathbf{c} \mid l), l=1 . . L\} .
$$

An important fact is that, in contrast to the classical colour separation scheme, we can now distinguish a large number $L$ of markers with only $C=2$ channels.

Making use of the fact that more marker types than detection channels can be used, a third dye, SRA617, is introduced, whose spectrum is shown in figure 3.12(a) with a red line together with the previously introduced dyes. Figure 3.12 (b) shows a two-dimensional histogram which illustrates the expected distribution of the photons of the three markers. The photon number distribution is assumed to be geometrical and the distribution to the channels 
(a)
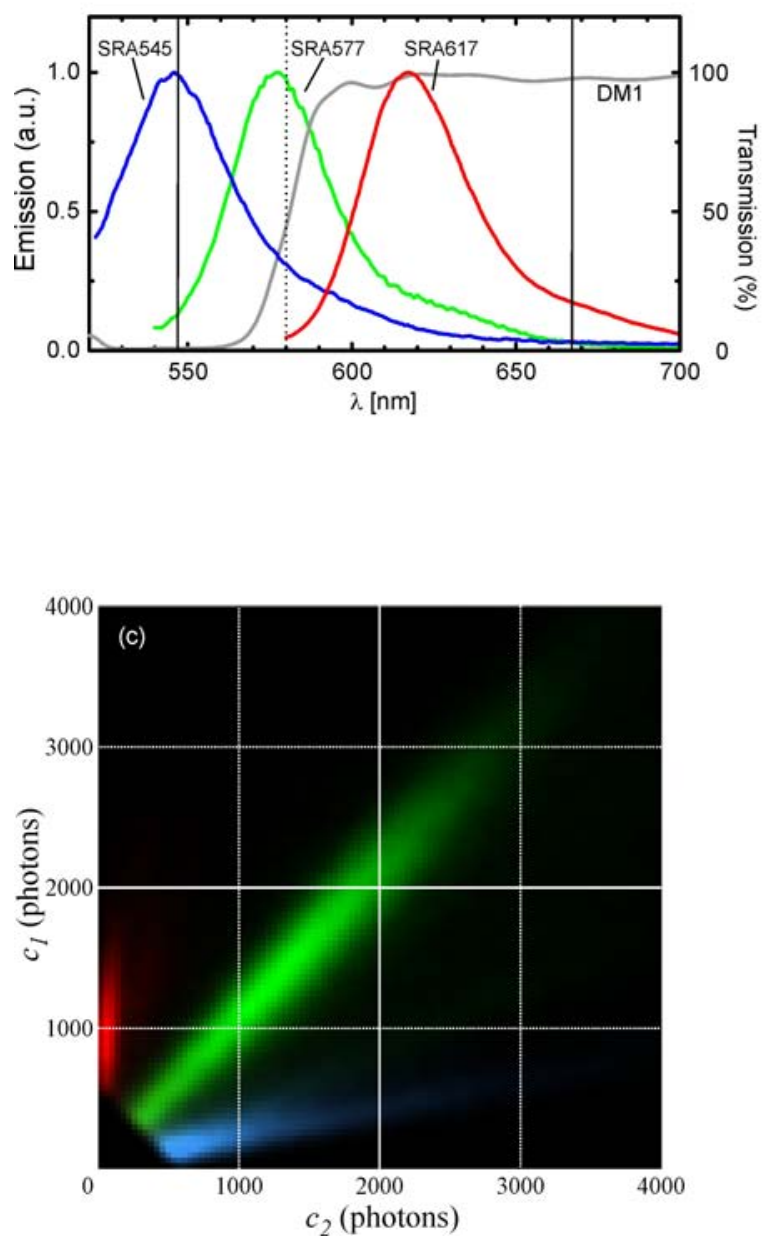
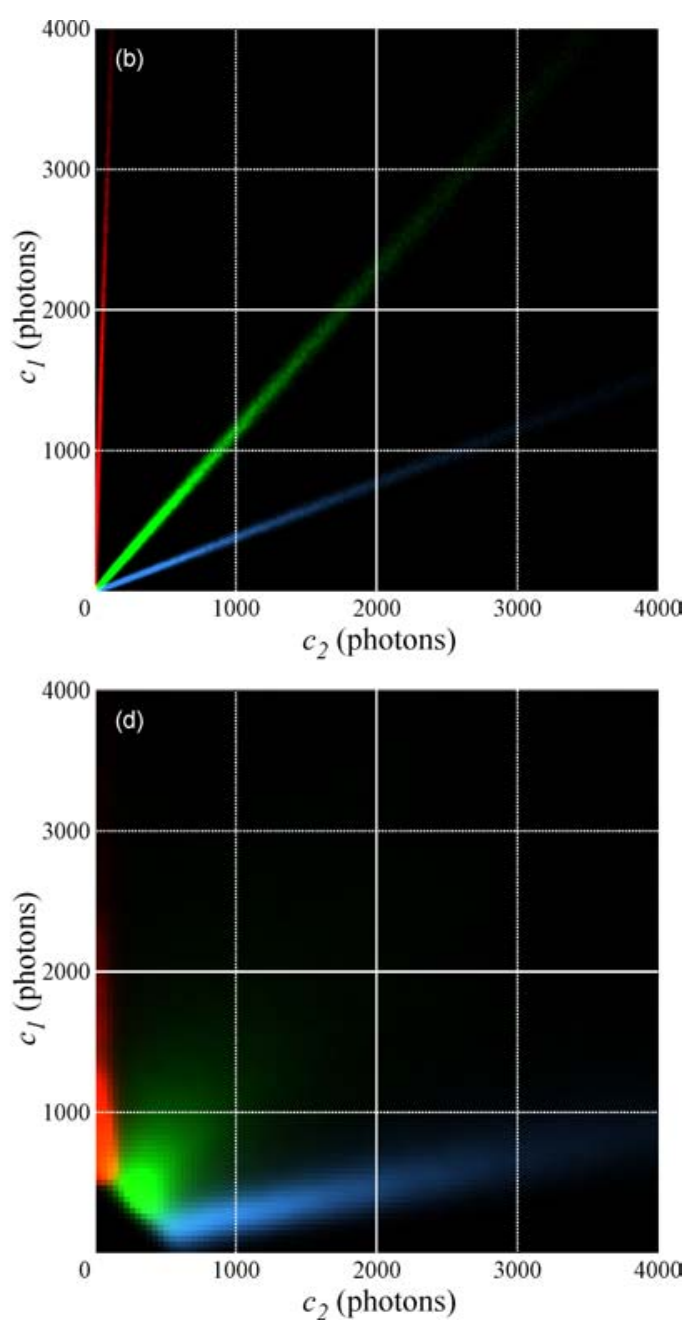

Figure 3.12: (a) Spectrum of markers SRA545 (blue), SRA577 (green) and SRA617 (red). The solid vertical lines at $547.5 \mathrm{~nm}$ and $667.5 \mathrm{~nm}$ denote the total detection window, the dotted line at $580 \mathrm{~nm}$ the border of the two channels used for the calculations. The solid grey line is the transmission of the actual dichroic mirror which performs the splitting of the channels in the experiment. (b) 2D-histogram of the photon distribution to the channels for the three dyes for a geometric distribution of the total photon numbers per burst and a binomial distribution to the two channels based on the spectra shown in (a). Note that for SRA617 (red), the probability for photon to end up in channel 1 has been artificially increased to 0.03 , otherwise the events could not be seen in the plot since they were too close to the edge. (c) Measured distribution histogram of the three dyes in Mowiol. (d) Measured distribution histogram of the three dyes in silica nanobeads with the same settings as in (c) but using a dichroic mirror shifted $10 \mathrm{~nm}$ to the red to compensate for spectral shift of SRA577 in that environment. 
binomial. The colour coding corresponds to figure 3.12 (a) and the number of events at a position $\left(c_{1}, c_{2}\right)$ is coded in shades of the respective colour. The signal from all three markers is located in regions closely around a straight line through the origin, whose slope is given by the ratio $c_{1} / c_{2}$.

The performance of the marker identification only depends on the separation of these regions. If the photon distributions overlap, false marker assignment will occur, which is equivalent to undesired cross talk in this case.

For photon numbers higher than some tens of photons, almost no overlap is obvious in figure 3.12 (b). Therefore, if only the stochastic variance of $\left(c_{1}, c_{2}\right)$ for a given $N=c_{1}+c_{2}$ was of importance, even a much larger number of marker types $L$ with only very little spectral separation could be used. For the case of figure $3.12(\mathrm{~b})$, the photon distributions of additional markers would just have to be located in the dark areas.

Unfortunately, the variance of the photon distribution to the two channels is mainly governed by other factors. The channel-splitting dichroic mirrors, for example, are often polarisation dependent. Also inhomogeneities in the sample lead to spectral variations in the signal from different regions. The distribution of the signals $\left(c_{1}, c_{2}\right)$ of all three markers in Mowiol is shown in 3.12(c) in blue for SRA545, green for SRA557 and red for SRA617. Shades of the respective colour represent the relative number of events for the given $\left(c_{1}, c_{2}\right)$. Again, all three markers form a distinct cloud, centred around a straight line through the origin whose slope is given by the ratio $c_{1} / c_{2}$. However, the width of the clouds is much larger than what was expected for the case of purely statistical noise. In addition to the three markers, a fourth one located spectrally between SRA617 and SRA577 can be included, but for even more markers, a third channel would have to be added to avoid strong overlap.

As already mentioned, the large width of the distributions is attributed to inhomogeneities in the sample. To demonstrate how environmentally dependent the signal of the markers is, 3.12 (d) shows the distribution of $\mathbf{c}=\left(c_{1}, c_{2}\right)$ for the same dyes but incorporated in silica beads. Note that the distribution of SRA577 is much wider and its brightness is much lower. On the other hand, SRA545 shows a higher brightness. The distribution of SRA577 was also shifted, therefore a different dichroic mirror has been used (see figure caption). It is thus recommended to record such a distribution histogram for each dye in the environment in which the final measurements will be taken in, instead of simply using the data from the recorded spectra and the maximum likelihood method described above.

If normalised such that $\sum M_{l}=1$, with $M_{l}$ the number of events recorded for each dye type $l=1 . .3$, the measured distributions plotted in figure 3.12 (c),(d) directly represent the probability distributions $P(\mathbf{c} \mid l)$. Therefore, for a given event with $\mathbf{c}^{\prime}=\left(c_{1}^{\prime}, c_{2}^{\prime}\right)$, the type $l^{\prime}$ can be determined according to equation 3.8 as

$$
l^{\prime}=\arg \max _{l}\left\{P\left(\mathbf{c}^{\prime} \mid l\right), l=1 . .3\right\} .
$$

The cross-talk $X_{k l}$ for each marker type $l$ is defined as the fraction of markers of types $l, l=1 . . L, l \neq k$ which were falsely recognised as type $k$ or in other words by the overlap of the distributions. Introducing

$$
A(k)=\left\{\mathbf{c} \mid \arg \max _{l}\{P(\mathbf{c} \mid l), l=1 . .3\}=k\right\}
$$


as the set of value pairs $\left(c_{1}, c_{2}\right)$ assigned to label $k$, it can be calculated as

$$
X_{k l}=\sum_{(c 1, c 2) \epsilon A(k)} P(\mathbf{c} \mid l)
$$

and thus be determined from the calibration measurements.

In order to reduce the cross-talk, the overlapping regions of the probability distributions can be disregarded. More specifically, those events $\mathbf{c}=\left(c_{1}, c_{2}\right)$ can be discarded, where the probability $P_{\text {max }}(\mathbf{c})=\max _{l}\{P(\mathbf{c} \mid l)\}$ that this event stems from the most likely marker type $l^{\prime}=\arg \max _{l}\{P(\mathbf{c} \mid l), l=1 . .3\}$ is not significantly higher than the probabilities $P(\mathbf{c} \mid l), l \neq$ $l^{\prime}$, that it stems from the other marker types $l \neq l^{\prime}$. Introducing a confidence estimator $C_{\text {conf } f}$, this can be achieved by excluding all events $\mathbf{c}$ where

$$
C_{\text {conf }}>\frac{\max _{l}\{P(\mathbf{c} \mid l)\}}{\sum_{l} P(\mathbf{c} \mid l)} .
$$

Interestingly, the crosstalk can thus be reduced at the cost of the total amount of events per image, which defines the signal range of the final image ('dynamic range').

Additional linear unmixing can still be applied on the final image.

\subsubsection{Markers}

Several different switchable rhodamine amide (SRA) markers have already been introduced in the preceding sections. It is indeed one of the interesting advantages of the SRAs that it is possible to easily change the properties of the fluorescent isomer, such as emission spectrum, lifetime, etc., by chemical modification of the fluorophore moiety of the molecule. The derivatisation of the carboxylate of a rhodamine with an aliphatic or aromatic primary amine does not significatively alter its emission properties (for example a redshift in the absorption and emission maxima of $5-10 \mathrm{~nm}$ is commonly observed for the amide derivative, as compared to the respective free carboxylate). Thus, similar markers for multicolour applications, differing in their emission spectra, can be obtained by starting from different rhodamines with the desired properties. In the experiments performed in the present thesis, four different marker types were used: SRA545, SRA552, SRA577 and SRA617. The chemical structures are presented in figure 3.13 (a).

Water soluble free acids and lithium or sodium salts were used for solution experiments or to cast polymer films. Amino reactive derivatives for each marker were prepared, except for SRA552, where a thiol reactive maleimide was produced. They were used to functionalise either silica beads or secondary antibodies. The emission spectra of markers SRA545 and SRA552 present a considerable overlap. The latter was used in replacement of the former for cellular measurements due to its considerably higher solubility in water which facilitates coupling to antibodies. The spectrum of the dyes is shown in figure 3.13. b).

All the markers prepared proved suitable for SMS microscopy. Independently of the amine used for the amide derivatisation, all resulting dyes present a sufficient switching-on 
efficiency in the near-UV region $360-380 \mathrm{~nm}$. Moreover, they can all be excited with green light. Thus only a single laser of $375 \mathrm{~nm}$ was used for activation, and another laser of $532 \mathrm{~nm}$ for excitation in all multicolour experiments combining up to three of the dyes shown in figure 3.13 .

(a)

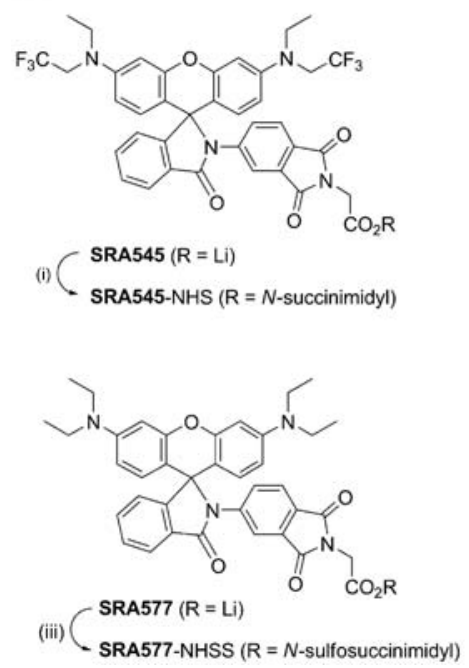

(b)
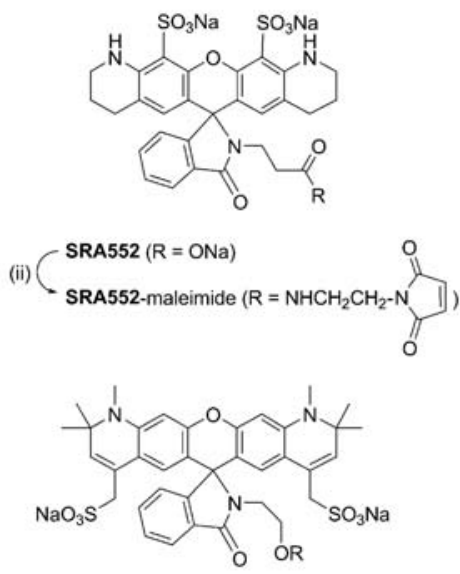

(iv) SRA617 (R=H)

SRA617-NHS (R = OCO-N-succinimidyl)

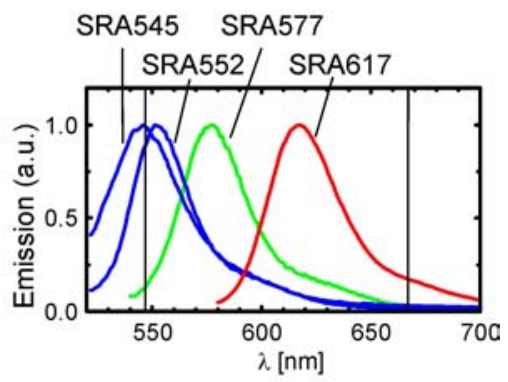

Figure 3.13: (a) Chemical structures of markers SRA545, SRA552, SRA577 and SRA617 as well as their reactive derivatives SRA545-NHS (i), SRA552-maleimide (ii), SRA577-NHSS (iii) and SRA617-NHS (iv) used for the experiments described later. All dyes can be activated using light of $375 \mathrm{~nm}$ and excited at $532 \mathrm{~nm}$. (b) Spectra of markers SRA545, SRA552 (both blue), SRA577 (green) and SRA617 (red). The solid vertical lines at $547.5 \mathrm{~nm}$ and $667.5 \mathrm{~nm}$ denote the total detection window.

\subsubsection{Imaging Setup, Registration and Type Assignment}

The setup used for the measurements is sketched in figure 3.14(a). The dichroic mirror DM2 splits the detection path into channel 1 for the photons with a longer wavelength and channel 2 for the photons with a shorter wavelength. Each detection path images the same area of the sample onto different parts of the camera. The imaged area is determined by a rectangular aperture with aspect ratio 2:1 which is placed in the image plane created by the objective and tube lens L4. The aperture is then imaged onto the upper and lower half of the camera CCD by channel 1 and channel 2, respectively, using lenses L3 in combination with L1 and L2. For both channels, a bandpass fluorescence filter was inserted. F1 in channel 1 had a transmission window from $592 \mathrm{~nm}$ to $667.5 \mathrm{~nm}$ and F2 in channel 2 had a transmission window from $547.5 \mathrm{~nm}$ to $622 \mathrm{~nm}$ (see figure caption for filter types).

Figure 3.14(b) shows a typical camera frame taken from a measurement with all three dyes incorporated in silica beads. The top half shows the image from channel 1, the lower half the image from channel 2. The dark horizontal line in the middle separates the two channels. All events where the signal in at least one channel is above the detection threshold are marked with circles and the corresponding event pairs from channel 1 and channel 2 are numbered. 

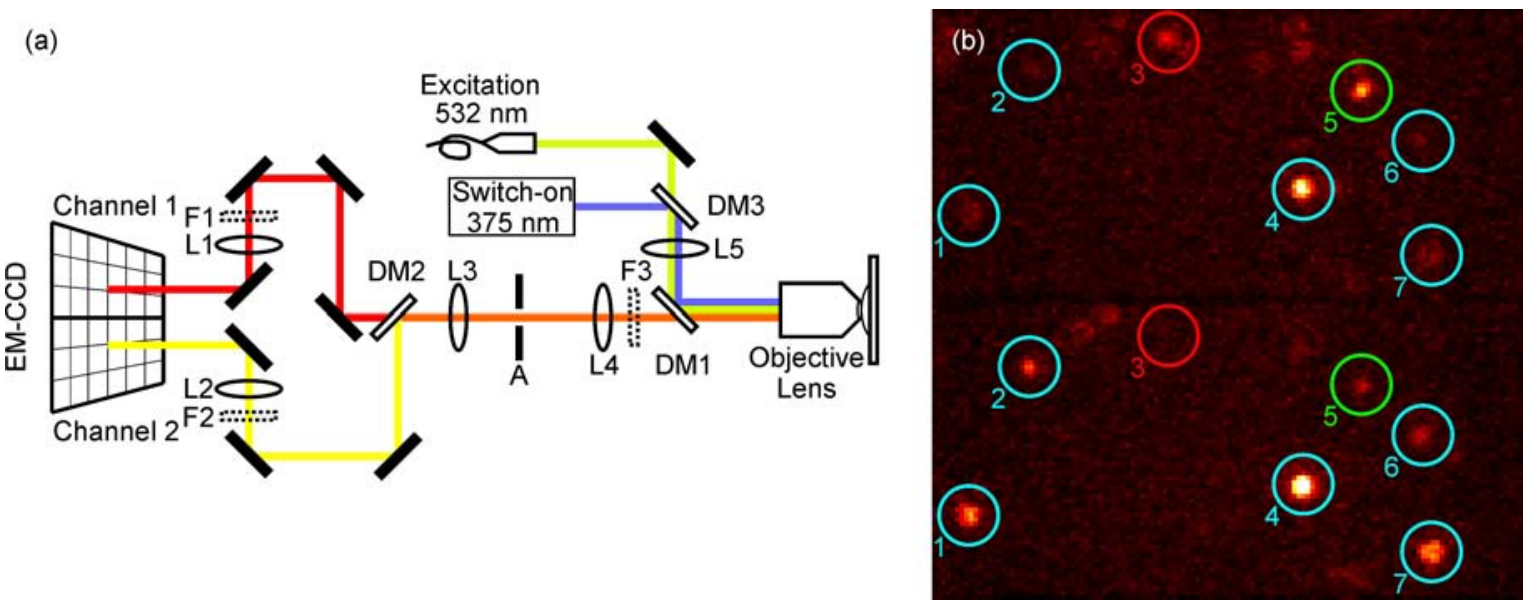

Figure 3.14: (a) Setup for dual-channel imaging. Excitation light preparation as described in figure 3.4. L1L5: Lenses with focal lengths $f_{1,2}=250 \mathrm{~mm}, f_{3}=150 \mathrm{~mm}$ and $f_{4,5}=80 \mathrm{~mm}$. DM1-3 are dichroic mirrors. DM1: z514rpc, splitting at $514 \mathrm{~nm}$, DM2: z580dcxr, splitting at $580 \mathrm{~nm}$ or z590dcxr splitting at $590 \mathrm{~nm}$ (see text), DM3: z375rpc. F1-3: Filters ET630/75M, FF01-585/75-25 and NF01-532U-25 (all filters from AHF, Tübingen, Germany). A: Square aperture. (b) Single sample frame. Note the dark horizontal line dividing channel 1 (top) and channel 2 (bottom). Circles denote the three marker species with the usual colour coding.

By eye, one can clearly recognise three different types of events. The events marked with the blue circles correspond to SRA545, they show brighter spots in the lower half than in the upper half. The event marked in green, corresponding to SRA577, shows slightly more photons in the upper half. The red event, from SRA617, shows no detectable photons in the lower half, almost all photons are directed to channel 1.

The initial localisation of the events is performed independently of the channels on the whole camera frame $(128 \times 128$ pixel $)$ as described earlier and the positions and photon numbers of all events are saved in a list. Events appearing in the upper half of the CCD, corresponding to pixel rows $1-64$ of the CCD chip, are attributed to channel 1 . The ones in the lower half, that is pixel rows $65-128$, are attributed to channel 2 . In order to match the event pairs from channel 1 and channel 2, which stem from the same molecule, a mapping function is needed. It transforms the spatial variables of channel 2 to the ones of channel 1, which serves as the reference channel. An affine transformation is used for this, which not only includes translation but also rotation and shear of the image in the lower half of the camera with respect to the one in the upper half of the camera. This mapping function is given by

$$
\mathbf{r}_{1}=\mathbf{A r}_{2}+\mathbf{t},
$$

where $\mathbf{r}_{2}=\left(x_{2}, y_{2}\right)$ denotes a position in channel 2 and $\mathbf{r}_{1}=\left(x_{1}, y_{1}\right)$ the corresponding position in channel 1 . The $2 \times 2$ matrix $\mathbf{A}$ and the translation vector $\mathbf{t}$ are determined by a calibration measurement. The accuracy of the mapping has to be significantly better than the average localisation accuracy of a single molecule event in order not to introduce an additional error source in the position determination. This can for example be achieved by imaging brightly fluorescing beads which emit into both channels and can be localised with 
an accuracy much better than the single molecule events. Alternatively, one uses the data of an actual SMS-measurement itself. One can average over a large number of frames from one measurement, yielding a mapping accuracy much better than the average localisation accuracy of the single molecule events. The advantage of the second method is that potential drift between the two channels during one measurement is automatically compensated for. Once the mapping transformation is found, that is $\mathbf{A}$ and $\mathbf{t}$, each detected marker of the whole measurement is associated with its counterpart in the respective other channel. This is achieved as follows: For each of the $P$ events at positions $\mathbf{r}_{1}^{i}, i=1 . . P$ in one camera frame which were registered in channel 1 , the $M$ events $\mathbf{r}_{2}^{j}, j=1 . . M$ from channel 2 from the same camera frame are scanned to check if any of them is at a position closer than a threshold of typically 0.2 times the FWHM of the detection PSF. If that is the case, this pair is considered to be the same event. The photon numbers $c_{1}, c_{2}$ for each channel are saved and the position $\mathbf{r}^{i}$ of each paired event $i$ is calculated as the weighted mean of the positions of the two channels

$$
\mathbf{r}^{i}=\frac{c_{i, 1} \cdot \mathbf{r}_{1}^{i}+c_{i, 2} \cdot\left(\mathbf{A} \mathbf{r}_{2}^{i}+\mathbf{t}\right)}{\left(c_{i, 1}+c_{i, 1}\right)}
$$

If an event only appears in one channel but is below the localisation threshold in the other, the algorithm simply records the number of photons at the position where the counterpart is expected by the mapping transformation and uses the position estimated in the one frame as the actual position.

The type assignment for each event pair $\left(c_{1}, c_{2}\right)$ is based on the previously recorded calibration histogram as described in section 3.2.1. For completion, the probability distribution histogram for the markers SRA545 (blue), SRA577 (green) and SRA617 (red) in silica is plotted again in figure 3.15 (a), this time with both histogram axes ranging to 8000 photons. Figure 3.15 (b) shows the confidence level for type recognition as given by equation 3.12 in colour coding. Figure 3.15 (c) then depicts the resulting valid identification regions of the three marker types for a confidence level of $C_{\text {conf } f} \geq 0.75$. All events $\left(c_{1}, c_{2}\right)$ ending in one of the coloured regions are assigned to the respective dye, all other events are discarded. The actually used regions of the probability distributions are thus truncated as shown in figure 3.15 (d).

\subsubsection{Experimental Results}

The ability to separate three marker types with only two channels was demonstrated using core-shell silica nanoparticles similar to the ones in section 3.1.6. The cores of these beads have a diameter of $\approx 70-90 \mathrm{~nm}$ and were stained with one of the marker types SRA545, SRA577 and SRA617 each. The unstained shell has a thickness of $\approx 15-20 \mathrm{~nm}$. The sample was prepared by spreading a dispersion of a mixture of the three bead types onto a glass cover slip and letting the solvent, ethanol, evaporate.

Figure 3.16 (a) shows a picture of the bead mixture in histogram view, taken with the discussed spectroscopic colour determination approach. In addition to the excellent resolution, the image shows a very good colour separation. Beads with SRA545 are shown in blue, those with SRA577 in green and the ones stained with SRA617 in red. In contrast to the 

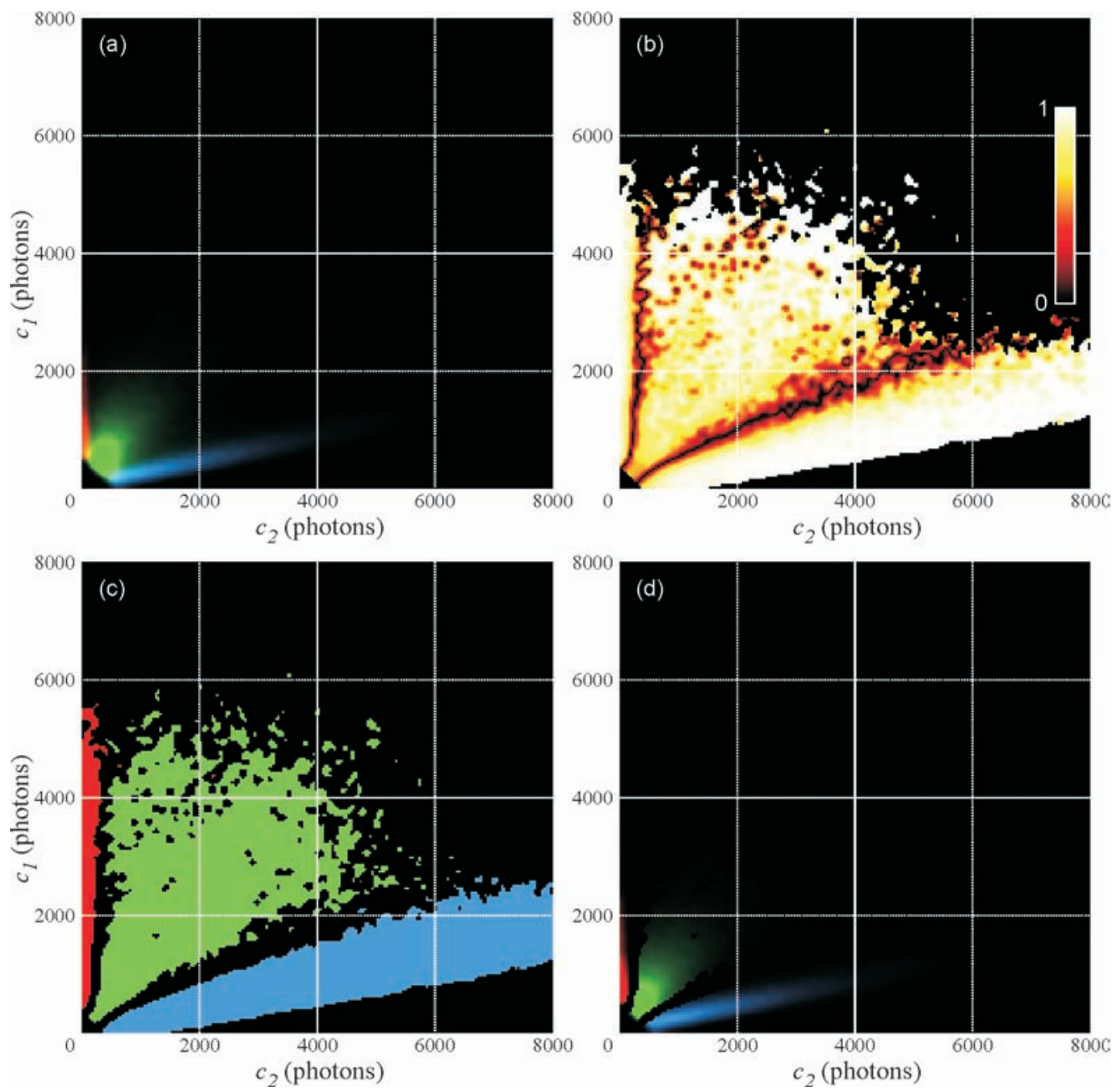

Figure 3.15: (a) Probability distributions for SRA545 (blue), SRA577 (green) and SRA617 (red) as in figure 3.12 (d) only up to 8000 photons for completeness. (b) Confidence levels of the type assignment as defined in equation 3.12 in colour coding. (c) Resulting valid identification areas for the three dyes. (d) Actually used regions of the probability distribution.

conventional wide-field image shown in 3.16(b), all beads can be resolved and the different bead types can be discerned with certainty. The conventional image has been obtained by adding up all 75000 frames, and plotting the resulting intensity distribution from channel 1 in shades of red and the one from channel 2 in shades of blue spatially overlapped. Figure 3.16 (c) shows an enlarged image of the region marked with the white box in figure 3.16(a). The individual beads can be well separated. Figures 3.16(d)-(f) depict the signal in the three type channels, the coloured rings mark the positions where the other beads of the respective types are located.

Figure 3.16(a) is composed of 161164 events assigned to SRA545, 135047 events to SRA577 and 47941 events to SRA617. The pixel size of the SMS-images in figures 3.16(a) and 3.16 (c)-(f) is $15 \mathrm{~nm}$. The dichroic mirror DM2 split the two channels at $580 \mathrm{~nm}$. The colour calibration data for the dyes in silica is the one shown in figure 3.12 (d) and figure 3.15 , re- 

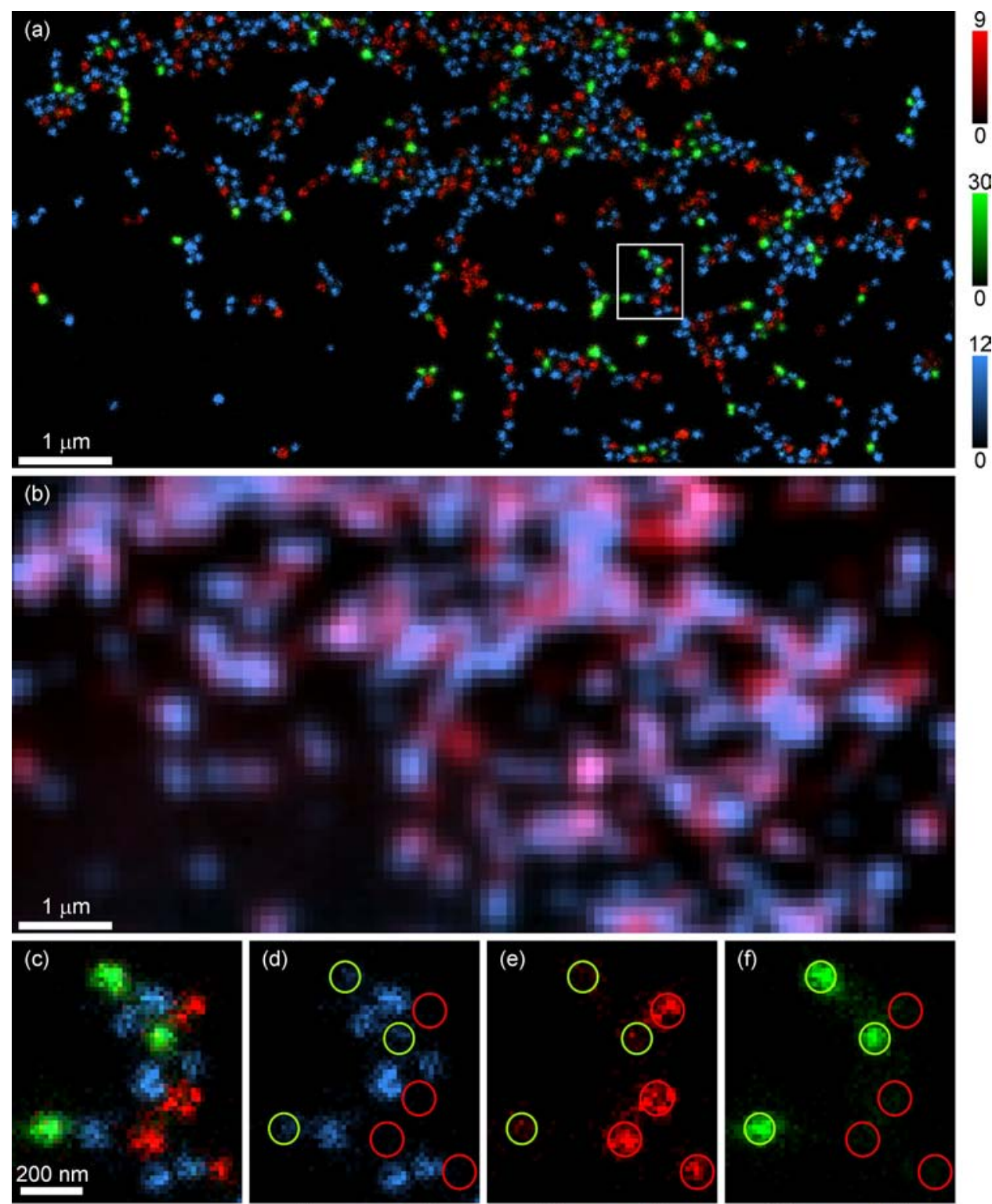

0

0

OE

30

Figure 3.16: Multicolour SMS-imaging. (a) SMS-image of silica core-shell nanobeads doped with the dyes SRA545 (blue beads), SRA577 (green beads) or SRA617 (red beads), respectively. The image has been taken using only two detection channels for the three colours (see text). The colourbars denote the colour-coding for the number of events per pixel. (b) Conventional wide-field image counterpart of (a), reconstructed by adding up all camera frames after background substraction and overlapping the two detection channels spatially. The signal from channel 1 is colour coded in shades of red, the signal from channel 2 in shades of blue. (c) Enlarged area as shown by the white box in (a). (d)-(f) The same area as in (c) is shown but the events recognised as SRA545, SRA577 and SRA617, respectively, are shown separately to visualise the cross-talk. The coloured rings show the location of the beads not present in the respective colour channels. The colour-coding of (c) is the same as denoted by the colourbars of (d)-(f). 
spectively, with the confidence level of $C_{\text {conf }}=0.75$ to reduce the cross-talk. The cross-talk matrix $\mathrm{X}$ can be calculated according to section 3.2.1. Since it is a-priori known that each bead comprises only markers of one type and the beads are well separated, the experimental crosstalk matrix $\mathbf{X}_{\text {exp }}$ can be directly estimated from this measurement. The columns of the cross-talk matrices represent the actual marker, the rows the marker it was assigned to, both in order of decreasing wavelength:

$$
\begin{aligned}
& \mathbf{X}=\left(\begin{array}{cccc}
0.88 & 0.07 & 0.00 \\
0.11 & 0.87 & 0.04 \\
0.01 & 0.06 & 0.96
\end{array}\right) \begin{array}{c}
\text { to SRA617 } \\
\text { to SRA577 } \\
\text { to SRA545 }
\end{array} \\
& \mathbf{X}_{\text {exp }}=\left(\begin{array}{cccc}
0.73 & 0.05 & 0.01 \\
0.23 & 0.87 & 0.08 \\
0.04 & 0.08 & 0.91
\end{array}\right) \begin{array}{c}
\text { to SRA617 } \\
\text { to SRA577 } \\
\text { to SRA545 }
\end{array}
\end{aligned}
$$

The cross-talk SRA617 $\rightarrow$ SRA577 (SRA617 falsely assigned to SRA577) is increased to 0.23 in $\mathbf{X}_{\text {exp }}$ as compared to the theoretically expected 0.11 in $\mathbf{X}$. The reason is a spectral drift of the marker SRA617 towards the blue during prolonged irradiation with the activation light. Therefore it overlaps more and more with the spectral region of SRA577 with increasing measurement time. To a much lesser extent, this is also true for SRA577 which shifts towards the spectrum of SRA545. In order to take this effect into account, a time dependent colour calibration would have to be applied. In the case of the image shown in figure 3.16(a), it was elected to restrict the plotted events to those appearing in the first 75000 measured frames, so that the resulting error is still acceptable.

For a conventional microscope, three colour channels would be necessary to separate the three colours of the beads. The expected cross-talk can be estimated by calculating the $K_{c l}$ of equation 3.3 for the spectra shown in 3.12 (a) using optimised cut-off wavelengths for the detection windows. The resulting cross-talk matrix is:

$$
\mathbf{K}=\left(\begin{array}{lll}
0.78 & 0.16 & 0.11 \\
0.22 & 0.72 & 0.43 \\
0.00 & 0.12 & 0.46
\end{array}\right)
$$

The columns are again the marker types in the order SRA617, SRA577, SRA545 and the rows are now the corresponding detection windows. This shows that the cross-talk would be much higher in the conventional approach, even with three colour channels. Linear unmixing has to be applied to extract the real concentrations $\mathbf{n}$ of the markers for each pixel:

$$
\mathbf{n}=\mathbf{K}^{-1} \mathbf{c}
$$

The choice of the optimal cut-off between the channels depends on factors such as the expected intensities and the white noise of the measurements [94]. To obtain matrix $\mathbf{K}$ of 


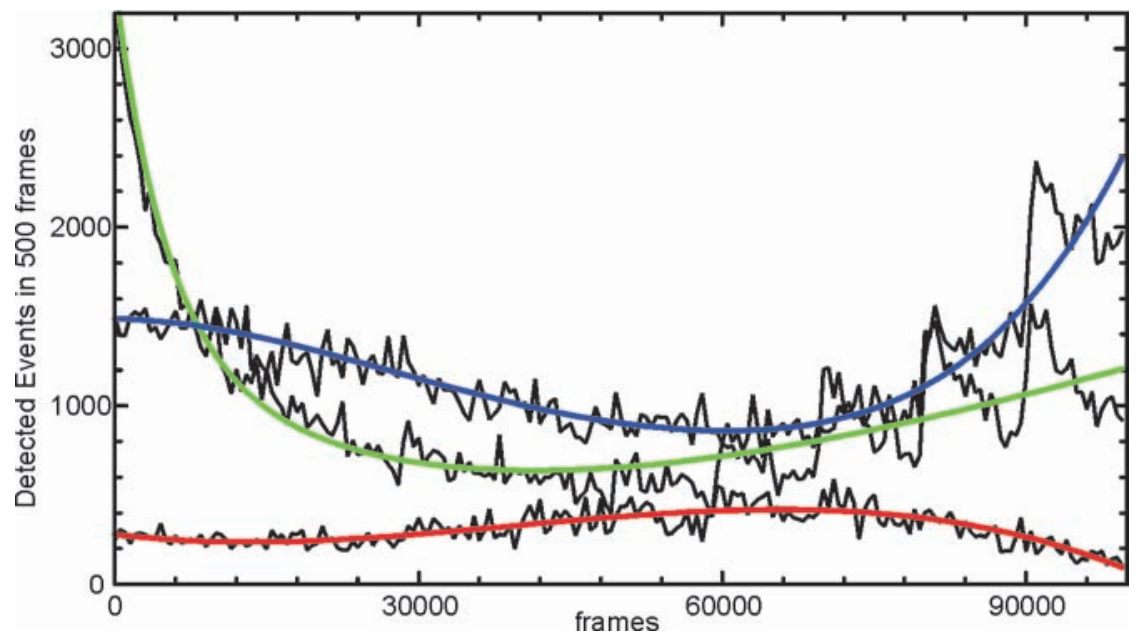

Figure 3.17: Number of activated dye molecules above threshold per 500 frames. SRA545: blue, SRA577: green, SRA617: red

equation 3.16, the cut-offs between the channels were chosen such as to maximise the smallest eigenvalue of the matrix $\mathbf{K}$, thus minimising the worst possible noise amplification. The resulting cut-off wavelengths are $563 \mathrm{~nm}$ and $608 \mathrm{~nm}$, while the whole detection window extended from $547 \mathrm{~nm}$ to $668 \mathrm{~nm}$. The smallest eigenvalue is 0.3 . Therefore, linear unmixing, like in equation 3.17, would improve separation but also amplify the noise threefold. Furthermore, time dependent spectral shifts might be detected easily in the single molecule approach, whereas they can remain hidden in the ensemble method. Also, for linear unmixing to be applied correctly, the distribution into the colour channels has to be estimated experimentally for each environment for conventional microscopy as well.

The activation probability of the markers differs. This can be seen in figure 3.17, where the number of detected events per 500 frames is plotted against the actual frame number for the measurement leading to the image displayed in figure 3.16. The colour coding is maintained. After 28000 frames, the activation with $375 \mathrm{~nm}$ light was started. One can see that the activation of SRA617 increases first, then SRA577 and finally SRA545, which has the least activation sensitivity. The spikes in the second half of the curves are due to the activation, which has not been increased continuously but in steps every 10240 frames. As usual, the activation light of $440 \mathrm{~W} / \mathrm{cm}^{2}$ was applied in pulses ranging from 0.1 to $50 \mu$ s. The excitation intensity was $32 \mathrm{~kW} / \mathrm{cm}^{2}$ and the frame rate was $100 \mathrm{~Hz}$.

In principle, such differences between the activation efficiencies can be used as an additional criterion for type assignment. It was, however, not used in this approach.

The single molecule based type recognition has also been applied to cellular samples. To this end, the microtubule and keratin networks of PtK2 cells were immunostained with SRA552 and SRA577, respectively and the cells were embedded in Mowiol. Keratin is a protein which forms an intermediate filament network. This network renders cell stability and flexibility. Keratin is for example found in large quantities in cells forming hair or fingernails. 
The resulting image is displayed in figure 3.18 (a) in histogram view. The microtubule network is displayed in blue, the keratin in green. The two structures are easily distinguishable. The signal associated with the respective dye types is displayed in figure 3.18 (b) and (c) for SRA577 and SRA552. Cross-talk from the other type due to false recognition is not obvious here. The single, isolated spots which can be observed, especially for the marker SRA577, are attributed to unspecific binding of the primary antibody.

The SMS-images of figure 3.18(a) to (c) are composed of 146400 events assigned to SRA577 and 276298 events assigned to SRA552. The pixel size corresponds to $20 \mathrm{~nm}$ in all three images. The dichroic mirror DM2 split the signal for the two channels at $590 \mathrm{~nm}$.

Figure 3.18(d) shows the reconstructed conventional image of the two overlapped channels. The signal from channel 1 is displayed in green, the signal from channel 2 in blue. Both channels are shown separately in figures 3.18 (e) and (f). From these three images the crosstalk becomes very obvious. One can easily see that both channels show significant signal from both structures. The fibres cannot be separated spatially nor can their type be determined. Linear unmixing is needed for dyes with spectra overlapping to such a large extent and only after that, the two structures are discernible, as shown in figure $3.18(\mathrm{~g})$. The cross talk in this case is

$$
\mathbf{K}=\left(\begin{array}{ll}
0.52 & 0.24 \\
0.48 & 0.76
\end{array}\right)
$$

In the case of single molecule based type recognition, linear unmixing is not necessary, the expected cross-talk from the calibration measurement is

$$
\mathbf{X}=\left(\begin{array}{cc}
0.94 & 0.05 \\
0.06 & 0.95
\end{array}\right) \quad \begin{gathered}
\text { to SRA577 } \\
\text { to SRA552 }
\end{gathered}
$$

In this case, no significant spectral shift was observed.

The colour calibration data for these dyes in cells is shown in figure 3.19(a). Figure 3.19(b) shows the confidence levels. The areas of valid type recognition are shown in figure 3.19 (c) and the residual regions after applying a confidence threshold of $C=0.75$ in figure 3.19 (d). The marker SRA552 emits a very high number of photons in this environment. Highintensity measurements at $55 \mathrm{~kW} / \mathrm{cm}^{2}$ and a frame rate of $50 \mathrm{~Hz}$ show that a mean photon number of $\bar{N} \approx 8000$ can be expected at a lower detection threshold of 400 photons. At these values, the electron multiplier (EM) gain of the camera has to be reduced significantly to prevent saturation effects. SRA577 shows a lower average photon number, here it is desirable to read out the signal with the highest EM-gain of the camera to reduce read-out noise. In order to be able to work in that regime, an excitation intensity of $19 \mathrm{~kW} / \mathrm{cm}^{2}$ has been applied and a frame rate of $200 \mathrm{~Hz}$, spreading some events of SRA552 over more than one frame. Figure 3.19(a) shows the photon distribution using these settings. The average photon numbers are 2600 and 1200, respectively. All events with less than 400 photons were rejected. The channel-splitting dichroic used in this measurements was the one depicted in figure 3.13 (a), the fluorescence filters were those mentioned in the figure caption of figure $3.14(\mathrm{a})$.

Activation pulses of $1 \mu \mathrm{s}$ to $1 \mathrm{~ms}$ were applied. Figure 3.20 shows the activation of the 

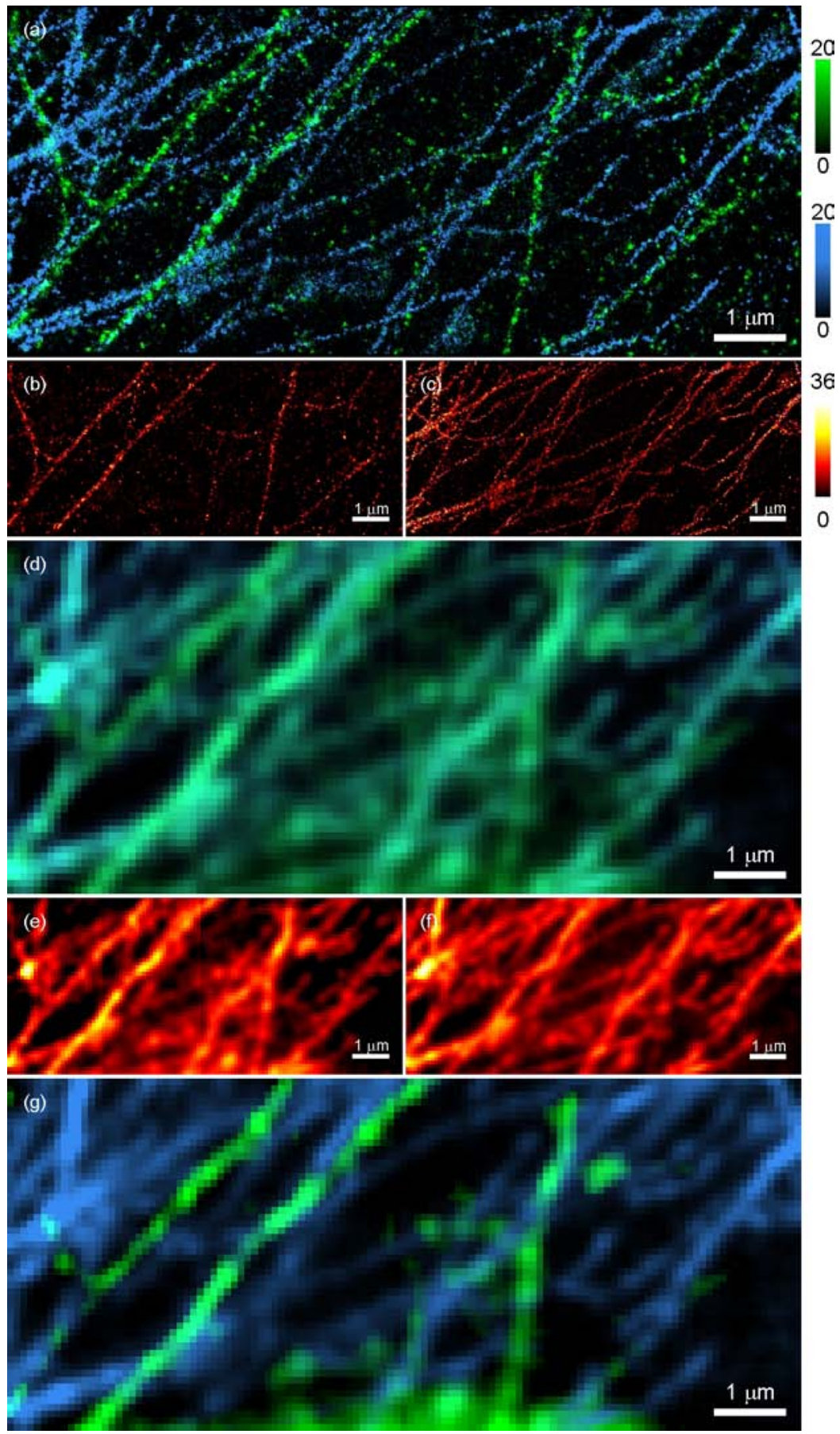

Figure 3.18: Dual-colour imaging of the microtubule and keratin network of a fixed PtK2-cell embedded in Mowiol. (a) The SMS image. The microtubule network (blue) is stained with SRA552, the keratin network (green) with SRA577. Both structures can be clearly distinguished and resolved spatially. The colourbars denote the colour-coding of the number of events per pixel. (b) All events assigned to marker SRA577, i.e. the keratin. The structure can be clearly seen. No cross-talk from the SRA552-stained microtubules is obvious. (c) All events assigned to marker SRA552, i.e. the microtubules. No cross-talk from the SRA577-stained keratin is obvious. (d) Reconstructed conventional wide-field image showing the signal from channel 1 (green, $590-669 \mathrm{~nm}$ ) and channel 2 (blue, $547-590 \mathrm{~nm}$ ) spatially overlapped. (e) Signal in channel 1. (f) Signal in channel 2. Note the large cross-talk obvious in figures (d)-(f). (g) Linearly unmixed wide-field image. Only now are the two structures discernible. 

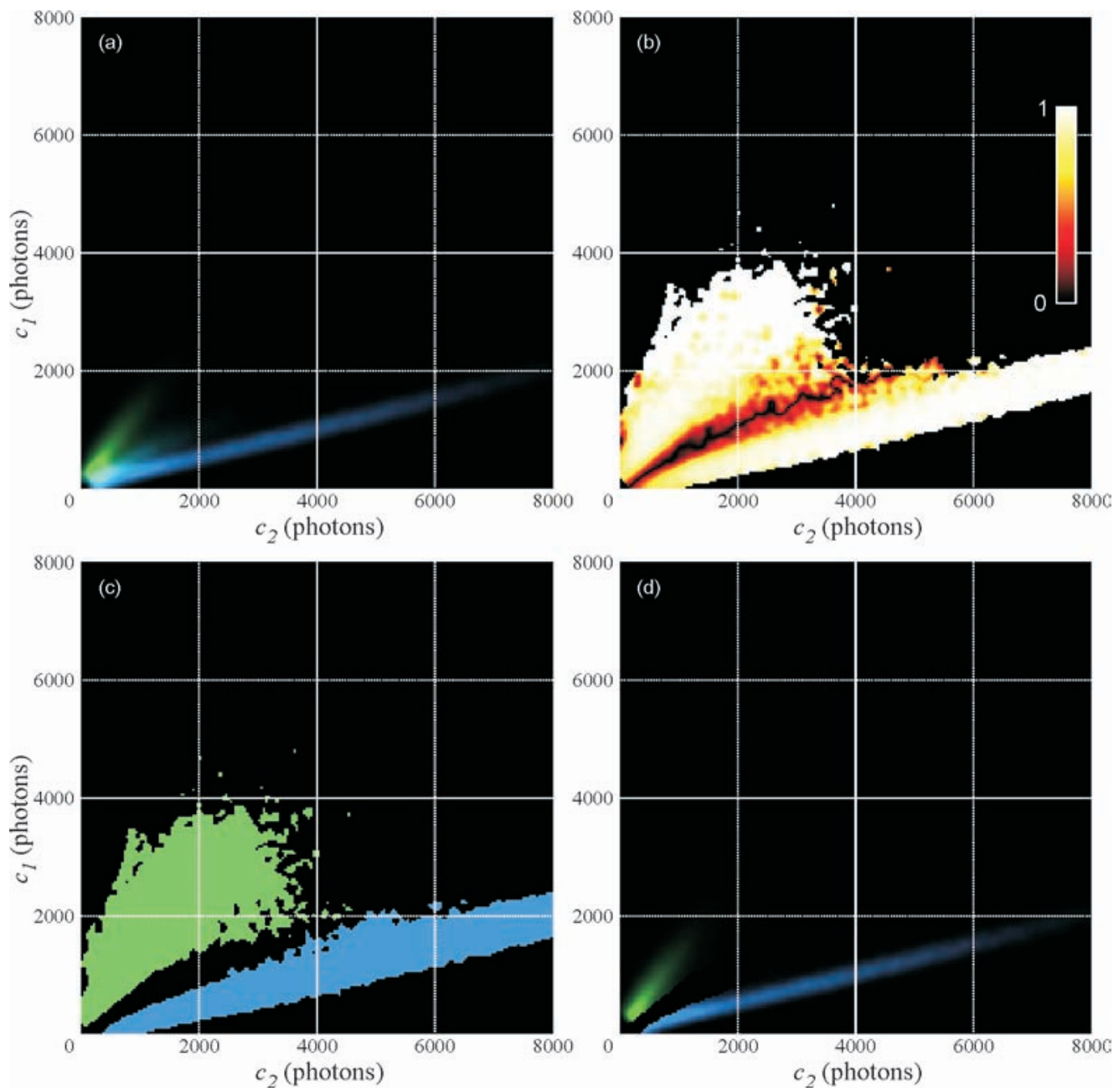

Figure 3.19: (a) Probability distribution of events $\left(c_{1}, c_{2}\right)$ for markers SRA552 (blue) and SRA577 (green) for the settings mentioned in the text. (b) Confidence levels for type assignment. (c) Areas of valid type assignment. i.e. where $C>0.75$. (d) Effectively used probability distribution area.

markers in the measurement for the image displayed in figure 3.18 as the pulse lengths of the activation light is increased, analogous to figure 3.17 .

\subsubsection{Experiments with Fluorescent Proteins}

The introduced colour separation scheme is naturally not restricted to organic dyes. In experiments with switchable fluorescent proteins, it was possible to distinguish two markers whose emission maxima are only $18 \mathrm{~nm}$ apart, which is practically impossible with conventional colour separation [74]. These fluorescent proteins, Dronpa and a new Dronpa-variant called bsDronpa belong to the so called reversibly switchable fluorescent proteins (RSFP). They can be excited by light around $476-488 \mathrm{~nm}$, which also induces a switch-off mechanism. Reactivation can be achieved by illumination with near-UV light in the range of $375-415 \mathrm{~nm}$. Since these molecules can be reversibly switched, they are also candidates 


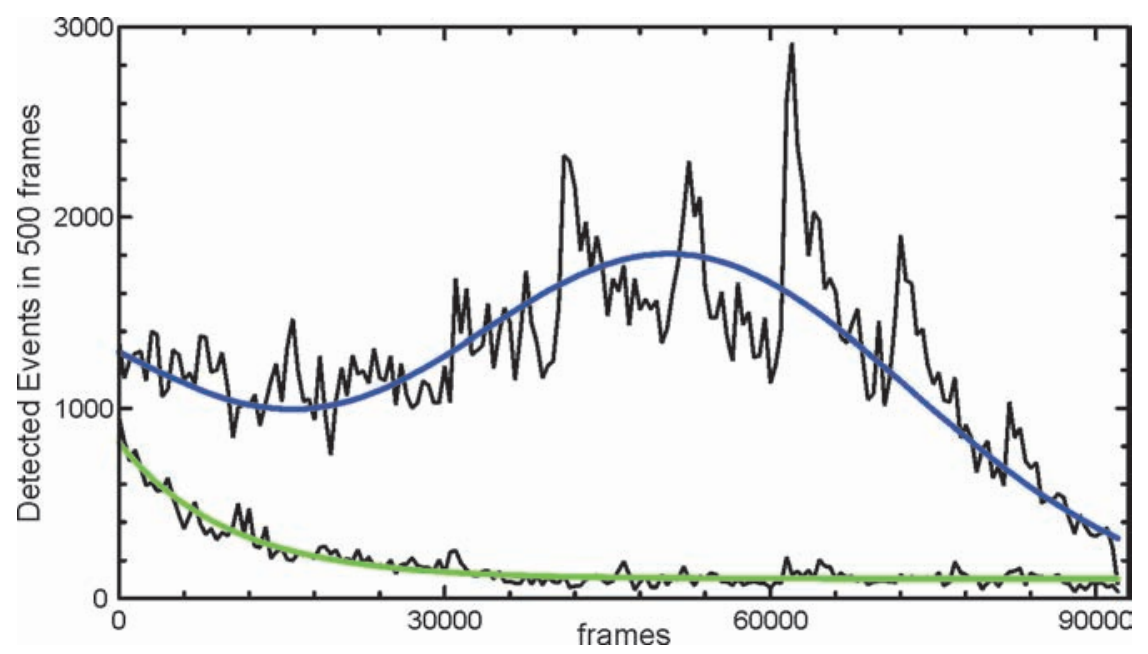

Figure 3.20: Activation of markers in the measurement for the image of figure 3.18 . Events registered as SRA552 are marked blue, events registered as SRA577 are marked green. The activation is measured in registered events per 500 frames. The signal spikes stem from the activation light whose intensity was increased stepwise.

for RESOLFT-type microscopy [13]. Here, however, they were used in a SMS-approach. The absorption (dashed line) and emission (solid line) spectra of the two proteins are shown in figure 3.21(a), in red for Dronpa and green for bsDronpa. The dotted black line denotes the cut-off of the splitting dichroic filter at $520 \mathrm{~nm}$ and the solid black lines the band of the total detection window. The distribution calibration measurement for the single molecule approach is shown in the upper left panel of 3.21 (b). The confidence values for colour assignment are shown in the upper right panel. The applied confidence threshold of $C=0.75$ led to the valid assignment areas shown in the lower left panel. $40 \%$ of the events are discarded with these settings. The resulting effective probability distributions are shown in the image in the lower right panel. This led to the experimentally estimated cross talk of $10 \%$ of Dronpa molecules wrongly assigned as bsDronpa (instead of $20 \%$ in the case of no confidence threshold) and $30 \%$ of bsDronpa wrongly assigned as Dronpa (instead of $40 \%$ ).

Figure 3.21(c) shows the SMS image in Gaussian view of a cryosliced, $200 \mathrm{~nm}$ thick section of an Escherichia Coli (E.coli) bacterium which expressed the new bsDronpa protein in its membrane. In comparison, the reconstruction of the conventional image is shown in Figure 3.21(d). The increase in resolution is obvious. The thickness of the membrane is measured as $50 \mathrm{~nm}$, which represents the resolution of the microscope with these proteins as markers. Figure 3.21(e) displays a cryosliced section of a mixture of E.coli cells, some of which expressed Dronpa and others bsDronpa in their membranes. Clearly the two species can be distinguished. This is not possible in the conventional image in figure 3.21(f), where the two channels are overlapped in the usual way. Red denotes channel 1 and green channel 2. Linear deconvolution did not yield a significantly different result.

These measurements were performed with the same setup as shown in figure 3.14(a) with only few changes. The excitation wavelength was changed to $476 \mathrm{~nm}$ supplied by a fibrecoupled Argon-Ion laser (Innova 70C, Coherent Inc.). The excitation light was also focussed 
(a)

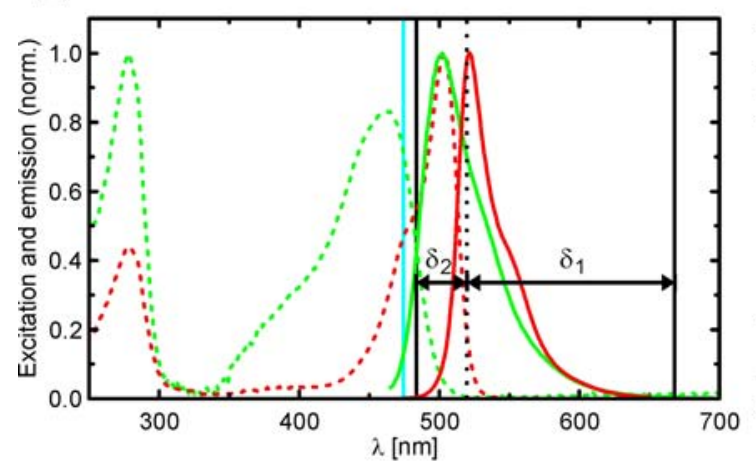

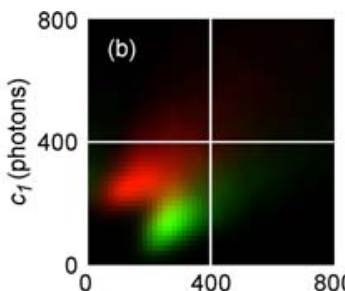

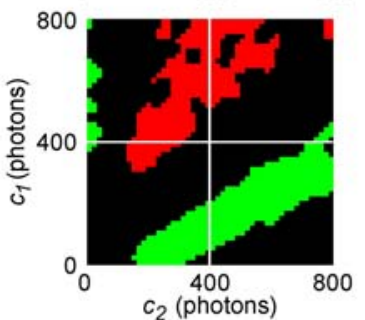

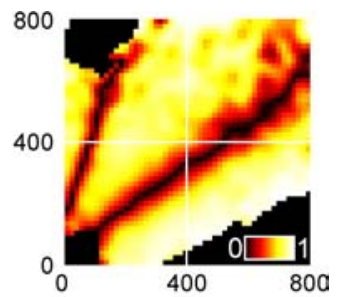

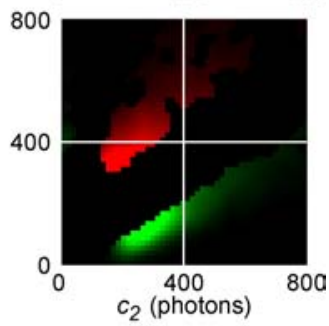

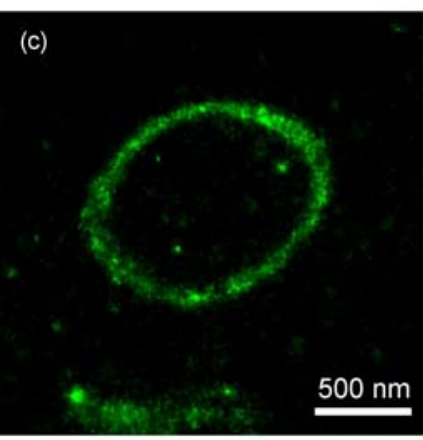
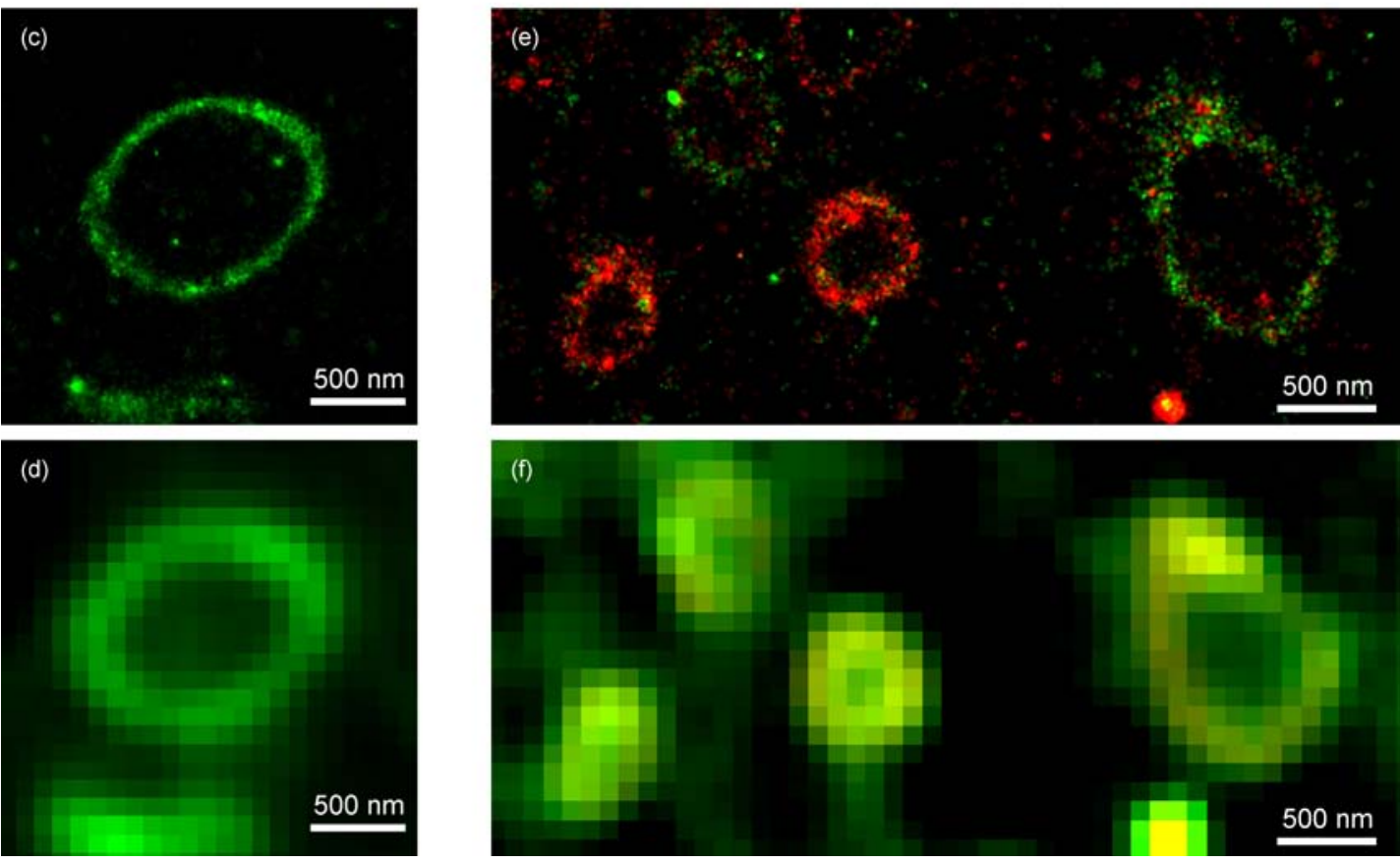

Figure 3.21: (a) Excitation (dashed line) and emission (full line) spectra of Dronpa (red) and bsDronpa (green). $\delta_{1}$ and $\delta_{2}$ denote the spectral window of detection channel 1 and channel 2, respectively. The turquoise line shows the excitation light at $488 \mathrm{~nm}$. The black solid vertical lines denote the total detection window, the dotted black line visualises the splitting wavelength of $520 \mathrm{~nm}$.(b) Upper left panel: Photon distribution in the two detection channels. The red area corresponds to signal from Dronpa and the green area to signal from bsDronpa. Upper right panel: Confidence levels for type assignment. Lower left panel: Areas of valid type assignment for a confidence level $\geq 0.75$. Lower right panel: Truncated photon distribution areas of (a). (c) SLM-image in Gaussian view of a $200 \mathrm{~nm}$ thick slice of an E.coli bacterium which expressed bsDronpa in its cell membrane. (d) Corresponding reconstructed wide-field image of (c). (e) Image of several sliced E.coli bacteria in Gaussian view. Red corresponds to signal from Dronpa, green to signal from bsDronpa. (f) Reconstructed wide-field-image of (e), where the two detection channels have been matched. Red denotes channel 1 (longer wavelength) and green denotes channel 2 (shorter wavelength). 
into the back focal plane of the objective, leading to a Gaussian intensity profile with a FWHM of $16 \mu \mathrm{m}$. The dichroic DM1 had to be exchanged with a z470rdc (AHF Analysentechnik) and the detection path had to be adjusted. Here, the splitting dichroic DM2 was exchanged with one splitting at $520 \mathrm{~nm}$ (z520dcxr, AHF) and bandpass filters with transmission windows from 494 to $570 \mathrm{~nm}$ for channel 1 and from $485 \mathrm{~nm}$ to $535 \mathrm{~nm}$ for channel 2 (both AHF) were introduced. The excitation intensity was on average $2.6 \mathrm{~kW} / \mathrm{cm}^{2}$ and the activation intensity $440 \mathrm{~W} / \mathrm{cm}^{2}$. The latter was applied in pulses up to $0.5 \mu$ s for the single colour imaging and up to $5 \mu \mathrm{s}$ for the dual colour case. The frame rate was $100 \mathrm{~Hz}$ and 40960 frames were recorded in both cases. The mean photon number in these measurements was 540 for the single-colour bsDronpa measurement, using a lower localisation threshold of $\approx 160$ photons, and 540 and 640 for bsDronpa and Dronpa, respectively, in the dual-colour measurement, using a threshold of 200 photons.

\subsubsection{Summary}

In this section, colocalisation of nanoscale structures was presented by exploiting only slight spectral differences in the emission spectra of the markers used to label the respective structures. With possible spatial resolutions of $\approx 15-20 \mathrm{~nm}$, this technique, like multicolour STED-microscopy, has the potential to close the gap between FRET-based colocalisation experiments and classical microscopy. The concept of using the spectral properties of single molecule events in order to discern the different marker types represents a methodical advancement over the classical multicolour imaging approach. The signal of the different markers is per se already unmixed and marker distinction based on the photon distribution into two channels can be performed reliably even for markers with strong spectral overlap due to the large number of emitted photons per burst and the resulting statistical certainty. The applicability was demonstrated for organic markers with a disparity in emission maxima of only $25 \mathrm{~nm}$ (SRA552 and SRA577) and fluorescent proteins whose emission maxima are only separated by $18 \mathrm{~nm}$.

This can be exploited such that multiple markers, whose spectra are so close together that they are all excitable by the same laser, can be used for staining. Together with the fact that only two detection channels are necessary to discern the multiple dyes, this makes the microscope setup very simple. Previous multicolour approaches were based on multiple excitation, activation and/or detection channels.

An additional advantage is the fact that all marker signals are recorded simultaneously and not sequentially for each marker type. This accelerates the image recording and eliminates the problem of relative movements between two differently marked objects between the imaging sequences.

Although the crosstalk between markers with overlapping spectra is already much lower in the single molecule approach than in the classical method, additional linear unmixing can still be applied at the cost of the signal to noise ratio. This is still possible if the number of markers exceeds the number of detection channels, unlike the conventional case. Another way to reduce the crosstalk is by introducing a confidence level for marker type discrimination. Thereby, cross-talk can be reduced significantly at the cost of the dynamic range of the image. In the case of sufficiently dense labelling, this can be compensated simply by 
increasing the measurement time. The only cost of this new kind of type recognition is the doubling of readout noise due to the two detection channels.

It is important to note that not only the emission spectrum but also other properties of the dyes such as fluorescence lifetime, anisotropy or FRET efficiency could be exploited. Also markers sensitive to their environment may be used to probe, for example, local $\mathrm{pH}$, temperature, rotational mobility, proximity to other markers and other physical or chemical parameters. The combination of single-molecule based nanoscopy and spectroscopy thus opens a whole new field of applications.

\subsection{SMS-Imaging with Conventional Markers using Ground State Depletion}

SMS-imaging needs a mechanism to toggle the markers between a non-fluorescent off-state and a fluorescent on-state in a controlled way. In order to achieve this, the attention in SMSmicroscopy has so far been on 'really' switchable markers, where the two states correspond to different conformational states or isomers of the molecules. This includes photoswitchable proteins such as EosFP [14], PA-GFP [16], etc., as well as organic Cyanine dyes both as activator-emitter-pairs [15] and as single photoactivatable labels [80, 95] and the switchable rhodamine amides discussed in this thesis. Although these markers have by now provided stunning images, they need dedicated activation or deactivation lasers, increasing the experimental effort [14, 15, 16, 25, 95]. Generally, the need for activatable compounds narrows the available dye types to a small subset of all fluorescent markers.

This section introduces the application of a broad range of conventional and commercially available organic dyes such as Rhodamine 6G, Atto532, Texas Red, etc., for SMS-microscopy. These dyes are known to be non-switchable. Almost every fluorophore and fluorescent protein, however, has long lived electronic dark states. This results in a typical blinking behaviour of the dyes, well known in single molecule spectroscopy. These inherent long-lived electronic dark states can be exploited and readily render conventional, non switchable dyes applicable for SMS-imaging. This concept is also known from RESOLFT-microscopy as Ground State Depletion (GSD) [19, 20, 30].

The conceptual and experimental simplicity of this approach is striking. The excitation and switch-off is performed with the same wavelength, and the spontaneous return of these dyes to their ground state even makes an activation laser obsolete, rendering the experimental setup very simple.

This SMS-microscopy variant is called GSDIM 'Ground State Depletion with Individual Molecule return' [29].

\subsubsection{Ground State Depletion}

Ground state depletion is a fluorescence switch-off mechanism which exploits the long lifetime $\tau$ of the first excited triplet state $\mathrm{T}_{1}$ of the fluorophore or other dark states $\mathrm{D}$ populated from $\mathrm{T}_{1}$ as shown in figure 3.22(a) [19, 20, 30]. If 'parked' in the dark state, the molecule is excluded from the process of excitation and emission between the ground state $S_{0}$ and the 
first excited singlet state $S_{1}$ and thus switched to the off-state (right of the dotted line). The corresponding on-state is the singlet $\mathrm{S}_{0}-\mathrm{S}_{1}$-system, where it can be cycled by the excitation light and fluoresce (left of the dotted line). Whenever a fluorophore is excited to $S_{1}$ with its lifetime $\tau_{f l}$, it can either relax to $\mathrm{S}_{0}$ by emitting a photon or it can undergo intersystem crossing (isc) and transfer to $\mathrm{T}_{1}$ with a probability $p_{i s c}$. A dye molecule under continuous illumination with the excitation light will therefore emit a photon burst with the mean number of photons $\bar{N}=1 / p_{i s c}$ before it will be taken out of the cycling process by entering $\mathrm{T}_{1}$. Assuming sufficiently high excitation light intensity and neglecting dark states other than $T_{1}$ or states populated from there [96], the residual fraction $\varepsilon$ of molecules in the on-state state is then

$$
\varepsilon=\frac{\tau_{f l}}{p_{i s c} \cdot \tau} .
$$

If $\tau$ is sufficiently large, almost all molecules can be transferred to $\mathrm{T}_{1}$. Only a very small fraction $\varepsilon$ of markers is left in the ground state $S_{0}$ from where they can undergo excitationemission cycles and emit photons, hence the name 'ground state depletion'. Since the access to the dark state happens stochastically via the excited singlet state $S_{1}$ of the marker, no switch-off light is needed. The molecules in the off-state transfer spontaneously back to $\mathrm{S}_{0}$ with the rate $1 / \tau$ and emit another photon burst with an average photon number $\bar{N}$, before they go again into a dark state or are irreversibly photobleached. For a sufficiently long measurement time, all molecules will therefore eventually return to the on-state and emit a photon burst and can thus be registered.

In order to yield a high number of photons per burst and thus a good localisation accuracy, $p_{i s c}$ is desired to be small. On the other hand, a large fraction of dyes has to be in the dark state in order to be able to image densely stained samples, therefore $\tau \gg \tau_{f l} / p_{i s c}$ is needed. For a typical $p_{i s c} \leq 10^{-4}$ and fluorescence lifetime $\tau_{f l}=1 \mathrm{~ns}$, we get

$$
\tau>\frac{\tau_{f l}}{p_{i s c} \cdot \varepsilon}=1 \mathrm{~ms}
$$

if more than $99 \%$ of the markers are desired to be in the dark state. The lifetime $\tau$ of a standard fluorescent dye is usually in the range of $\tau=10^{-3}-100 \mathrm{~ms}$ [19], depending on the dye and the environment. In addition, $T_{1}$ also serves as a gateway to other dark states with similar or even longer $\tau$ of $1-10^{4} \mathrm{~ms}$ [96]. Therefore, GSDIM seems indeed possible under the right conditions.

Two methods of increasing the lifetime $\tau$ of the dark states beyond the millisecond range were applied: Oxygen reduction leads to long triplet lifetimes since oxygen serves as a triplet quencher. Therefore, aqueous media with addition of glucose, glucose-oxidase and catalase were used. Glucose-oxidase is an enzyme which uses glucose to bind oxygen, whereas catalase is an enzyme which decomposes the resulting toxic hydrogen peroxide. The second method was embedding the samples in PVA. PVA constrains the oxygen diffusion, leading to a prolonged triplet lifetime, and furthermore introduces additional dark states [96]. Both media are standard microscopy embedding media. Details on their composition can be found in the appendix A.2 
(a)

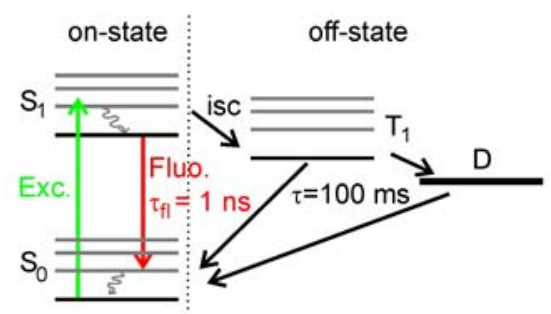

(b)
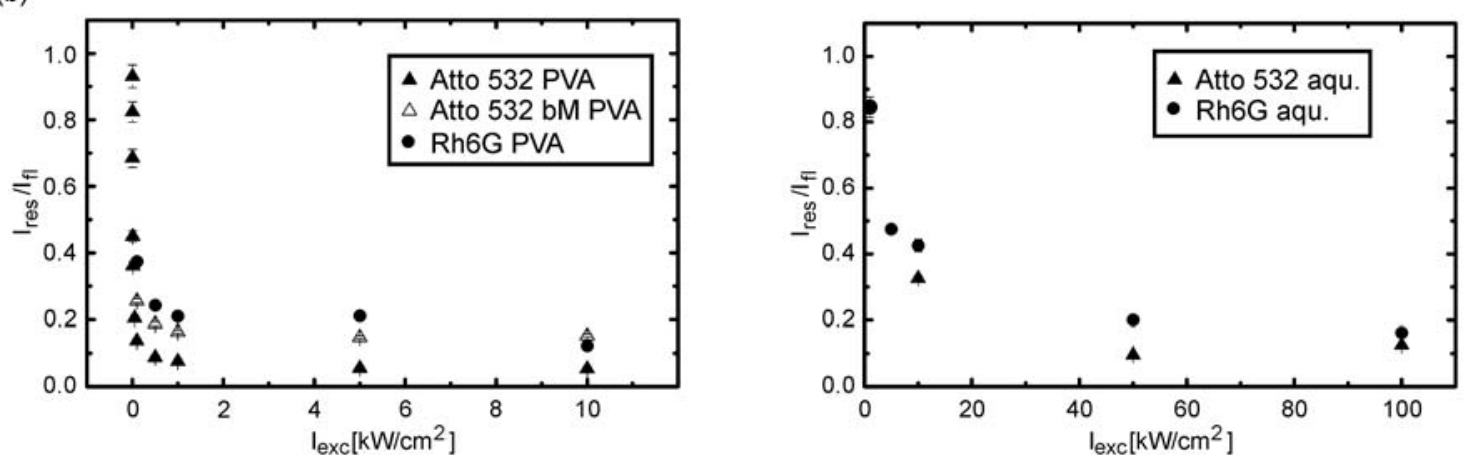

(c)
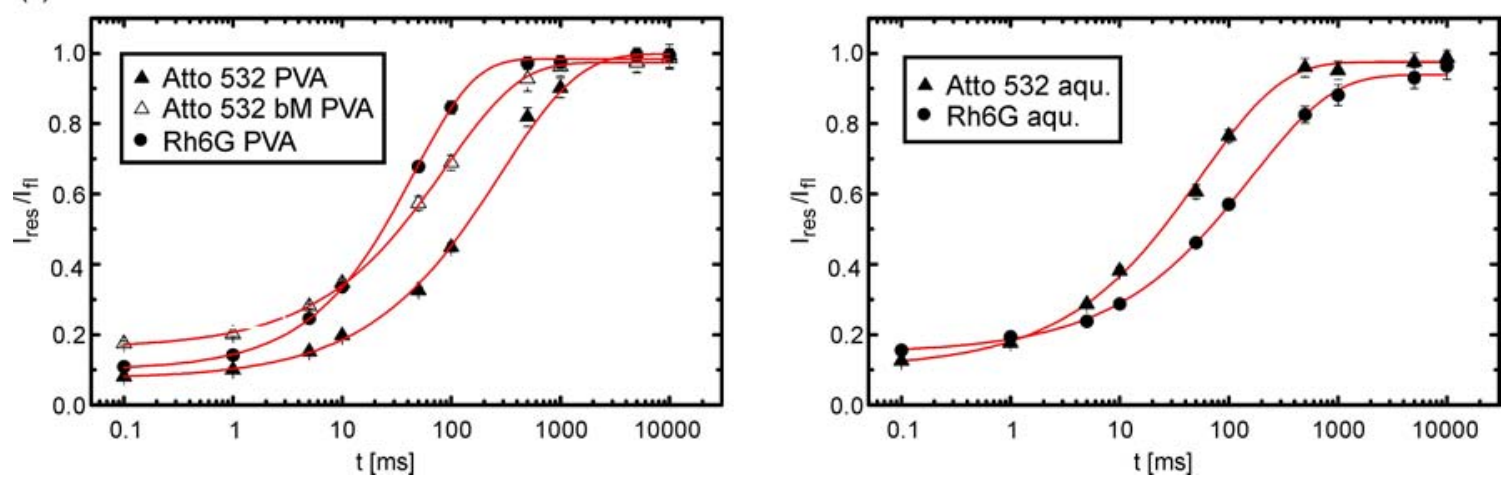

Figure 3.22: (a) Jablonski state model of a conventional dye. The on-state of the dye is the singlet $\mathrm{S}_{0}-\mathrm{S}_{1}$ System of the dye (left from dotted line), the off-state is the long lived triplet state $\mathrm{T}_{1}$ and other dark states populated therefrom (right of dotted line). (b) Left side: Residual fluorescence fraction of Atto532, Atto532 with addition of $\beta$-mercapto-ethanol (bM) and Rh6G in PVA. Right side: Residual fluorescence fraction of Atto532 and Rh6G in aqueous buffer with addition of glucose-oxidase and catalase. The data was taken in a probe-pump-probe measurement (see text) in dye ensembles. (c) Left side: Recovery from dark state for Atto532, Atto532 with addition of $\beta$-mercapto-ethanol (bM) and Rh6G in PVA. Right side: Recovery from dark state for Atto532 and Rh6G in aqueous buffer with addition of glucose-oxidase and catalase. The dye was bound to the microtubular network of PtK2-cells by immunostaining. The red lines are fits to the data (see text). The data has been measured in a dye ensemble in a probe-pump-probe experiment (see text). Data courtesy of Hannes Bock [29]. 
Figure 3.22(b) shows the effect of ground state depletion for two commercial dyes, Rhodamine 6G (Rh6G, circles) and Atto532 (full triangles). The left panel depicts the relative fluorescence $I_{\text {res }} / I_{f l}$ of an ensemble of the respective dye molecules embedded in PVA, after pumping the markers into the dark state with a $10 \mathrm{~ms}$ pulse of the excitation light of intensity $I_{e x c} . I_{f l}$ corresponds to the signal if all dyes are in the on-state and $I_{\text {res }}$ to the the residual fluorescence after the light pulse which transfers the markers to the dark state. The right panel shows the same measurement with the dyes in aqueous buffer with addition the glucoseoxidase and catalase. The fluorescence decreases with increasing intensity of the light pulse, showing that an increasing fraction of markers is pumped to the dark state. Equation 3.20 is valid in the region where the signal $I_{r e s} / I_{f l}$ is saturated, that is for $I_{e x c}>5 \mathrm{~kW} / \mathrm{cm}^{2}$ in the case of PVA and $I_{e x c}>80 \mathrm{~kW} / \mathrm{cm}^{2}$ in the case of the aqueous buffer. In this region, $I_{r e s} / I_{f l}$ directly corresponds to $\varepsilon$. Values of $\varepsilon$ below $10 \%$ were obtained.

The measurements were performed as probe-pump-probe measurements, where firstly the reference signal $I_{f l}$ was taken with a low intensity probe pulse of the excitation light $\left(100 \mathrm{~W} / \mathrm{cm}^{2}\right.$ for $0.5 \mathrm{~ms}$ ). Secondly, the dyes were pumped to their triplet state with a $10 \mathrm{~ms}$ pulse of the excitation light with the intensity $I_{\text {exc }}$ as plotted on the abscissa. Directly after that the residual fluorescence $I_{\text {res }}$ was probed again by a second probe pulse identical to the first one.

Note that due to the nature of the pump-probe measurements, the values obtained for $\varepsilon$ are overestimated. This is because during the first probe pulse, where all markers are initially in the on-state, dyes are already pumped into the triplet state. Therefore, the fluorescence signal is already decreasing during the probing. Since the measured reference signal $I_{f l}$ corresponds to the signal averaged over the whole probing time, it is underestimated as compared to the signal expected for all dyes in the on-state. Additionally, during the second probe pulse, the dyes are starting to return due to the low intensity of the probe pulse, resulting in an overestimated signal for $I_{\text {res }}$.

The dark-state lifetime was also measured by probe-pump-probe measurements. A stained sample containing a large amount of markers in the excited region was first probed with a $0.5 \mathrm{~ms}$ pulse of the excitation light with an intensity of $100 \mathrm{~W} / \mathrm{cm}^{2}$, yielding the fluorescence signal $I_{f l}$. A high intensity excitation pulse $\left(10 \mathrm{~kW} / \mathrm{cm}^{2}\right.$ for samples in PVA and $100 \mathrm{~kW} / \mathrm{cm}^{2}$ for aqueous solution) of $1 \mathrm{~ms}$ pumped the markers to the dark state. Subsequently, after varying times $t$, the residual fluorescence $I_{\text {res }}$ was tested with a second probe pulse with the same parameters as the first one. The resulting normalised fluorescence signal $I_{\text {res }} / I_{f l}$ is plotted versus time $t$ in figure 3.22 (c) for the commercial dyes Atto532 (triangles) and Rhodamine 6G (Rh6G, circles). The embedding media were PVA in the left plot and aqueous solution in the right plot. The plots show indeed a $\tau$ of several tens to a hundred milliseconds.

Addition of the triplet quencher $\beta$-mercapto-ethanol (bM) accelerates the recovery and reduces the fraction of dark molecules, which is shown by the open triangles in the left panels of figure 3.22(c) and 3.22(b), respectively. This indicates that the dark state is indeed the triplet or at least triplet-mediated. The time dependence of the recovery follows a stretched exponential

$$
I_{\text {res }}(t)=I_{f l}\left(1-\exp \left[-(t / \tau)^{\alpha}\right]\right)
$$

with exponents $\alpha=0.6-0.8$ significantly deviating from the expected mono-exponential 


\begin{tabular}{|c|c|c|c|}
\hline Fluorophore & Environment & $\varepsilon$ & $\tau[\mathrm{ms}]$ \\
\hline \multirow{2}{*}{$\mathrm{Rh}_{6 \mathrm{G}^{1}}$} & PVA & 0.10 & 45 \\
\hline & aqueous & 0.13 & 170 \\
\hline Rh110 & PVA & 0.19 & 1.5 \\
\hline $\mathrm{Rh}_{123^{2}}$ & PVA & 0.10 & 17 \\
\hline Rh-sart $3 b^{1}$ & PVA & 0.07 & 290 \\
\hline Rh-sart $3 \mathrm{f}^{1}$ & PVA & 0.04 & 390 \\
\hline \multirow{2}{*}{ Atto532 1} & PVA & 0.05 & 230 \\
\hline & aqueous & 0.10 & 60 \\
\hline Atto565 3 & PVA & 0.06 & 190 \\
\hline Alexa $488^{2}$ & PVA & 0.10 & 10 \\
\hline Oregon Green $^{2}$ & PVA & 0.04 & 7 \\
\hline Texas Red $^{2}$ & PVA & 0.05 & 5 \\
\hline FITC $^{2}$ & PVA & 0.22 & 0.5 \\
\hline Bodipy $^{2}$ & PVA & 0.06 & 6 \\
\hline $\mathrm{GFP}^{2}$ & aqueous & 0.05 & 50 \\
\hline EYFP $^{2}$ & aqueous & 0.05 & 50 \\
\hline \multirow{2}{*}{ Citrine $^{2}$} & aqueous & 0.10 & 50 \\
\hline & microtubule (Map2) & 0.15 & 1000 \\
\hline PhiYFP $^{2}$ & aqueous & 0.03 & 40 \\
\hline
\end{tabular}

Table 3.1: Values for the dark state population $\varepsilon$ and recovery times $\tau$ for different dyes and environments. All values obtained from ensemble measurements. The applied excitation-switch-off wavelengths were ${ }^{1}: 532 \mathrm{~nm}$, 2: $488 \mathrm{~nm}^{3}: 561 \mathrm{~nm}$. Data courtesy of Hannes Bock [29]

$(\alpha=1)$ recovery, which indicates more than just one involved dark state. The red lines in figure 3.22(c) are the fits to the data according to equation 3.22. The results for $\tau$ are listed in table 3.1 .

The values for the residual fluorescence and the recovery times for the dyes mentioned above and a number of additional standard markers are listed in table 3.1 .

It is noticeable that the experimental values for $\varepsilon$ of a few per cent are two to three orders of magnitude higher than expected from the estimation in equation 3.20 with $\tau$ on the order of tens to hundreds of milliseconds. One explanation is that so far light-induced reverseintersystem-crossing of the electrons from the dark state via higher excited states and subsequent relaxation to the ground state has been neglected. This 'back-pumping' to the ground state is very efficient when using light in the near-UV-range as shown in figure 3.23 (a) for $405 \mathrm{~nm}$ light. This is especially true in PVA. Also light of $671 \mathrm{~nm}$ shows an effect and increases the fluorescence significantly, as can be seen in figure 3.23 (b). Therefore it is very likely that the excitation-switch-off light of $488 \mathrm{~nm}, 532 \mathrm{~nm}$ and $561 \mathrm{~nm}$ used for the respective dyes in table 3.1 shows the same effect and thus reduces the fraction of molecules in the off-state. The off-state is therefore in fact a photostationary state. Although this is disadvantageous in the sense that the effect limits the maximum staining density of the sam- 

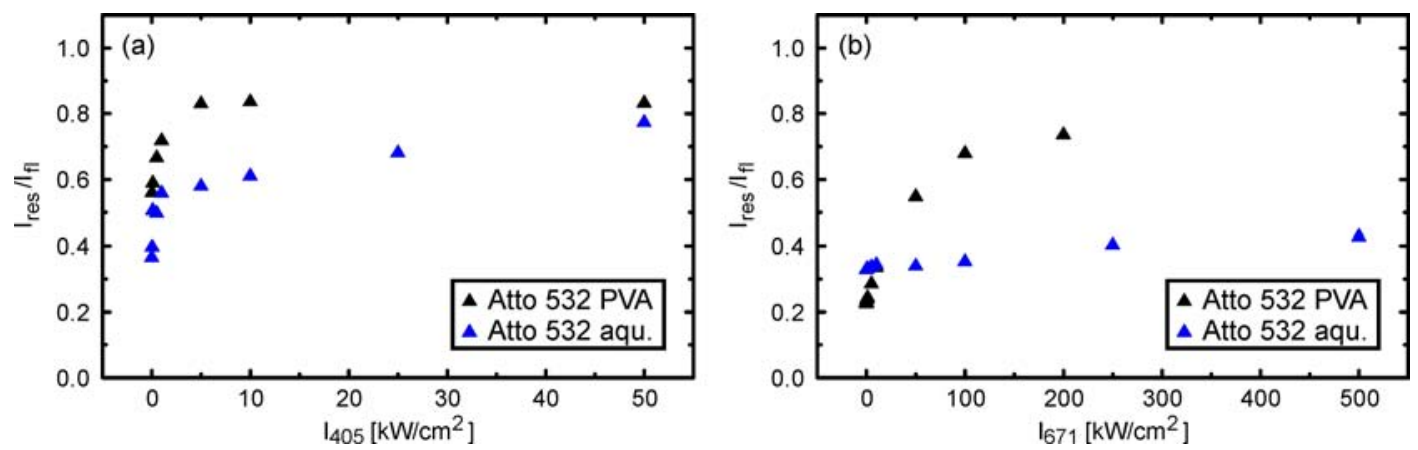

Figure 3.23: Acceleration of the return of the Atto532 fluorophores from the dark states by illumination by light of $405 \mathrm{~nm}$ in (a) and $671 \mathrm{~nm}$ in (b). The black triangles show the data for PVA, the blue triangles in aqueous buffer with glucose-oxidase and catalase. The measurement was performed in probe-pump-probe mode. First, $I_{f l}$ was measured with a probe pulse of the $532 \mathrm{~nm}$ excitation light of $100 \mathrm{~W} / \mathrm{cm}^{2}$ for $0.5 \mathrm{~ms}$. The the markers were pumped into the dark state with an $532 \mathrm{~nm}$ excitation pulse of $10 \mathrm{~ms}$ with an intensity of $1 \mathrm{~kW} / \mathrm{cm}^{2}$ for the case of PVA and $100 \mathrm{~kW} / \mathrm{cm}^{2}$ for the aqueous solution. The second probe pulse with the same settings as the first one followed $10 \mathrm{~ms}$ after the pump pulse, during which the back-pumping light was activated. The delay of $10 \mathrm{~ms}$ is the reason why the values for zero intensity for the back-pumping light differ from the values for maximum switch-off. Data courtesy of Hannes Bock [29].

ples for SMS-microscopy, it can be also used to enhance the speed of the measurement by illumination with light of for example $375 \mathrm{~nm}$ when the rate of molecules spontaneously returning to the ground state drops as more and more molecules are photobleached. This is, however, not compulsory.

\subsubsection{Staining Density}

Of the four mandatory attributes of an SMS-marker stated in section 1.5.6, three are fulfilled for a large variety of conventional markers which are commonly used in confocal and wide-field microscopy: Firstly, due to the low probability $p_{i s c}<10^{-4}$ to enter the dark state, a large burst of photons is emitted before switch-off, enabling high localisation precision. Secondly, the fraction of molecules in the on-state can be controlled by the intensity of the excitation light or by applying additional activation light if necessary. Thirdly, a perfect signal contrast between the bright and dark state is ensured. Only the point concerning the switch-off ratio seems problematic given the seemingly high amount of several percent of dyes left in the on-state. It is therefore worthwhile to consider the meaning of this value.

In each PSF-sized characteristic area $\mathrm{A}_{c}$ of $\approx 300 \mathrm{~nm}$ in diameter, only one marker may be active per frame. Therefore, given a residual amount of $5 \%$ of the dyes left in the onstate, and assuming that all of them emit photon bursts above the detection threshold, only 20 markers may be within this area at most. Each dye can usually be imaged several times with GSDIM before it photobleaches. Given the according localisation precision, it will thus produce a signal pattern in the final image approximately corresponding to a Gaussian of, for example, $25 \mathrm{~nm}$ width. Therefore, imaging isolated straight fibres for example will return smooth high resolution images, since twenty stochastically distributed spots of $25 \mathrm{~nm}$ within a length of $300 \mathrm{~nm}$ form a rather smooth line. If several fibre strands are present within $\mathrm{A}_{c}$ 


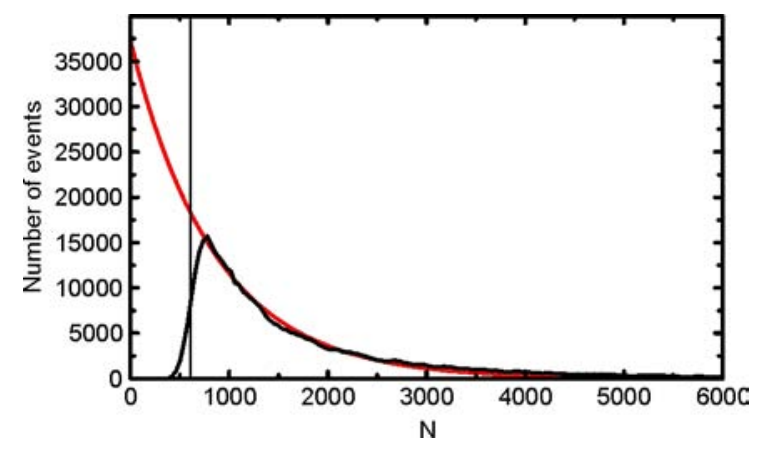

Figure 3.24: Histogram (black line) of the numbers of photons per event for the Atto532 markers of the measurement shown in figure 3.25 (a). The vertical depicts the lower photon threshold of $\approx 600$ photons. The red line is an exponential fit to the histogram in the range $N \geq 740$. The region $N \leq 740$ is extrapolated.

or other more detailed objects, the image will appear spotty.

However, since the number of photons per event follows a geometrical distribution, a large fraction of dyes in the on-state produces only events with very few photons before the dyes are returning to the dark state. These events are below the detection threshold and only attribute to background noise. The fraction of detectable markers is therefore lower than the fraction of dyes in the on-state, that is, $\varepsilon$. Note that the molecules are typically generating several bursts before photobleaching, therefore they will still be detected throughout the measurement, even if some events are below the threshold. No spatial information will therefore get lost. The experimental situation concerning the maximum applicable marker density is thus more favourable than suggested by the values for $\varepsilon$ of table 3.1. These values have been measured in dye ensembles and therefore directly represent the total fraction of markers in the on-state.

As an example, the photon distribution of the Atto532 markers of the measurement of figure 3.25(a) shown in section 3.3.3 is displayed in the histogram of figure 3.24 with the black plot. The events were binned in intervals of 40 photons. $n_{\text {det }}=414441$ events were detected in total, the lower cutoff of $T \approx 600$ photons (vertical black line) is given by type assignment confidence interval applied in the measurement. The geometric distribution can be approximated with an exponential distribution $f(N)=A \cdot \exp \left[-N / \bar{N}_{\text {geo }}\right]$. Fitting the histogram with $f(N)$ yields the parameters $A \approx 37165$ and the mean number of photons for the distribution $\bar{N}_{\text {geo }} \approx 858$. The fitting was performed in the range $N \geq 740$. The fitting function is shown with a red line, the region $N \leq 740$ is extrapolated. The total number of molecules which were in the on-state during this measurement is thus

$$
n_{t o t}=n_{d e t} \cdot \frac{\int_{0}^{\infty} f(N) d N}{\int_{T}^{\infty} f(N) d N}=\frac{n_{\text {det }}}{\exp \left[-T / N_{\text {geo }}\right]} \approx 937000
$$

Therefore, in each frame only a fraction of $\approx 0.44$ of all molecules in the on-state has been detected in this particular measurement. 


\subsubsection{Imaging and Results}

A significant practical aspect of using standard fluorophores for SMS-imaging is the fact that, in contrast to photoactivatable dyes, all marker molecules are initially in their on-state when commencing the experiment. This is advantageous since firstly the sample can be found more easily and secondly a conventional pre-image can be taken which may serve as an abort criterion for the SMS-measurement.

In order to start the SMS-measurement, all but the desired subset of molecules in the on-state have to be transferred to the triplet dark state. This is realised by irradiation with excitation light of high intensity. If the density of activated dyes in the photostationary state is still too large, which may happen for densely stained samples, the excess dyes can be photobleached.

To demonstrate the applicability of GSDIM, the microtubule network as well as the peroxisomes of PtK2-cells were immunostained with Atto532 and Atto565. Peroxisomes are vesicles which discharge toxic substances from the cell. They also play a role in the decomposition of fat.

The cells were embedded in PVA by putting a drop of 1\%-PVA solution on the glass coverslip on which the cells are growing and spincoating it for $20 \mathrm{~s}$ at $3000 \mathrm{rpm}$ (see appendix A.2 for details). No further embedding medium was used. The microscope setup was similar to the one shown in figure 3.14. After mounting and prebleaching for roughly $10 \mathrm{~s}$ with the excitation light of $532 \mathrm{~nm}$ at an intensity of $115 \mathrm{~kW} / \mathrm{cm}^{2}$, the imaging process was started. Figure 3.25(a) shows a dual-coloured image of the tubular network immunostained with Atto532 (green) and the peroxisomes immunostained with Atto565 (red) in histogram view. The colour separation was performed as described in section 3.2. The upper right corner shows the reconstructed conventional wide-field image after linear unmixing of the two channels, which was mandatory for the conventional image due to the small disparity of the emission peaks of the two dyes of only $40 \mathrm{~nm}$. Figure 3.25(b) shows the same kind of structures but with inverse staining, that is the microtubules are immunostained with Atto565 and the peroxisomes with Atto532. Again the reconstructed and linear unmixed conventional image is shown in the upper right corner. The difference in resolution can be seen very obviously along the borderline of the two image parts. The GSDIM-images feature an effective resolution of $<30 \mathrm{~nm}$, which has been determined by measuring the width of single, isolated spots which probably stem from single unspecifically bound antibodies.

Both images were taken using a frame rate of $100 \mathrm{~Hz}$. Figure 3.25 (a) is based on 72000 frames and was taken with an illumination intensity of $I_{e x c}=20 \mathrm{~kW} / \mathrm{cm}^{2} .82000$ frames at $I_{\text {exc }}=10 \mathrm{~kW} / \mathrm{cm}^{2}$ were taken for figure 3.25 (b). The crosstalk between the two dyes amounts to less than $10 \%$. The total number of events amounted to 414441 assigned to Atto532 and 39694 to Atto565 in figure 3.25(a) and 29819 assigned to Atto532 and 176443 to Atto565 in figure 3.25 (b). The mean photon number of the registered events was about 1600 for both dyes in both images after applying a lower photon threshold of $T \approx 600$ photons. The pixel size of the GSDIM-images is $20 \mathrm{~nm}$. Due to the fact that the smallest features in the images are on the order of $50 \mathrm{~nm}$, considering the size of the microtubules of $25 \mathrm{~nm}$ plus the primary and secondary antibodies added for the staining, this pixel size samples the image sufficiently, while still providing a significant number of events registered per pixel 

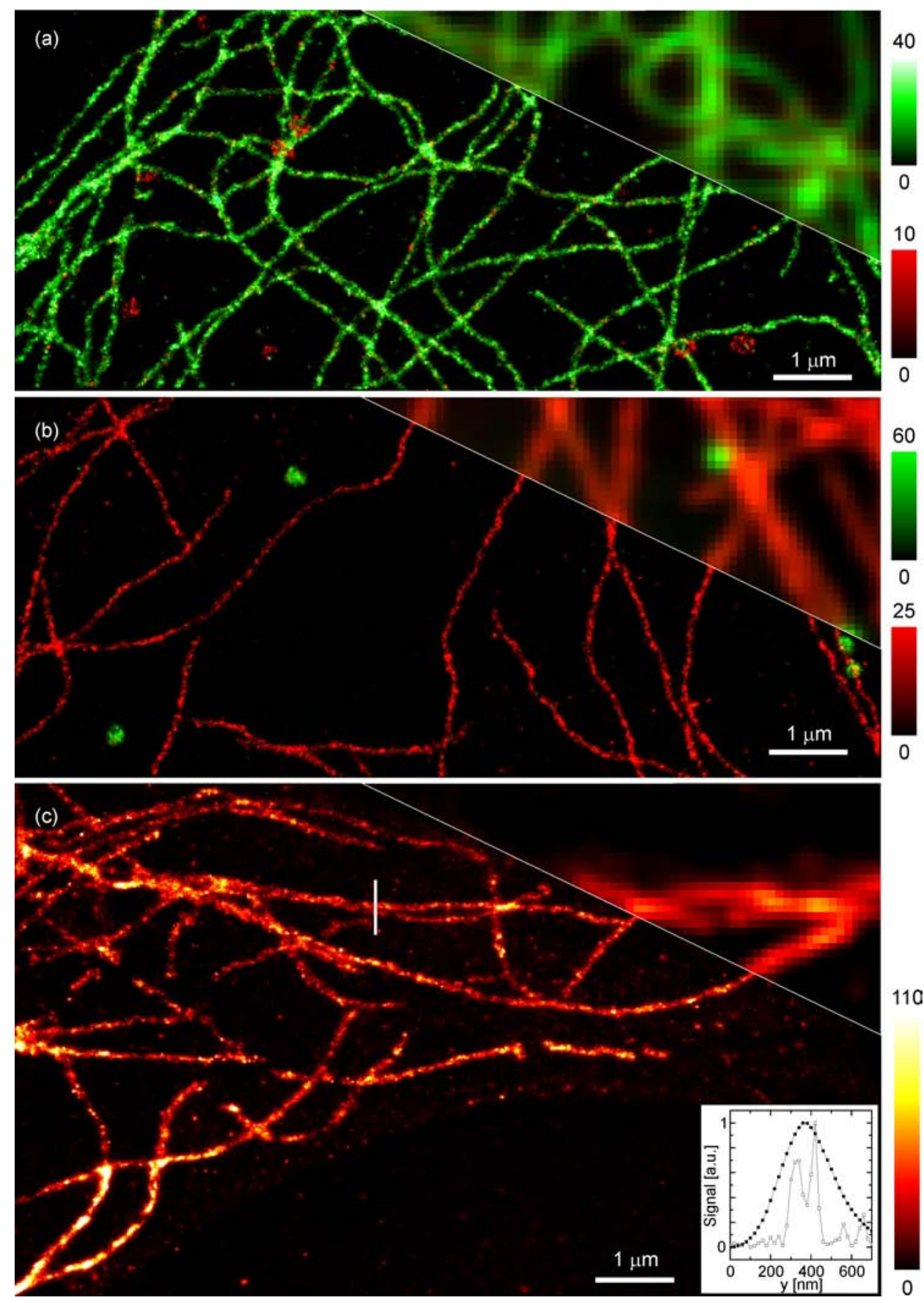

Figure 3.25: GSDIM imaging of cells. (a) Image of the tubular network and peroxisomes of fixed PtK2 cells in histogram view. The tubular network is immunostained with Atto532 (green), the peroxisomes with Atto565 (red). The sample is embedded in PVA. The upper right corner shows the conventional image obtained by summing up all the camera frames after background substraction. (b) Image of the same kind of structures as in (a) but with inverse staining, that is, the tubular structure is immunostained with Atto565 (red) and the peroxisomes with Atto532 (green). (c) Tubular network of a PtK2 cell immunostained with Rh6G displayed in colour coding in histogram view. The sample was embedded in aqueous solution. The inset shows the line profile as denoted by the white line in (c). The open circles show the line profile of the SMS-image, the filled circles the interpolated line profile of the conventional image. The colour bars on the right side of panels (a)-(c) denote the colour coding for the registered events per pixel. 
[23, 81]. The resolution estimates on single antibodies was performed on images with $10 \mathrm{~nm}$ pixel size in order to provide sufficient sampling. The image acquisition was accelerated by irradiation with light of $375 \mathrm{~nm}$ of intensity $440 \mathrm{~W} / \mathrm{cm}^{2}$ in between frames for up to $100 \mu \mathrm{s}$ from frame $\approx 20000$ and up to $300 \mu$ s from frame $\approx 10000$ onwards for figure 3.25 (a) and 3.25 (b), respectively.

In a second step microtubules of PtK2-cells were immunostained with Rh6G and the cells were embedded in TRIS-buffer containing glucose, glucose-oxidase and catalase to remove the oxygen $(50 \mathrm{mM}$ TRIS, ph $7.5,10 \mathrm{mM} \mathrm{NaCl}, 0.5 \mathrm{mg} / \mathrm{ml}$ glucose oxidase (G2133, Sigma, St. Louis, MO, USA), $40 \mu \mathrm{g} / \mathrm{ml}$ catalase (106810, Roche Applied Science, Mannheim, Germany) and 10\% (w/v) glucose). Figure 3.25(c) shows the SMS-image with the reconstructed wide-field image in the upper right corner. Imaging was performed with a frame rate of $200 \mathrm{~Hz}$ and an illumination intensity of $115 \mathrm{~kW} / \mathrm{cm}^{2}$ of the excitation and switch-off light of $532 \mathrm{~nm}$. The resolution was again estimated to be below $30 \mathrm{~nm}$. No $375 \mathrm{~nm}$ light was applied in this experiment, since the photobleaching of Rh6G in this environment is low. The number of events per frame is therefore only decreasing slowly and each marker could be imaged multiple times, leading to a high dynamic range of the image. 61000 camera frames were taken for this image, it consists of 870699 registered events with a mean photon number of $\approx 1600$, where the lower photon threshold was 400 . The pixel size is $20 \mathrm{~nm}$.

In a further step towards live-cell imaging, the applicability of GSDIM in the cell culture medium 'Dulbecco's Modified Eagle Medium' (DMEM) has been tested. The integrin- $\beta$ 3 protein of fixed human U373MG glioma cells was immunostained using Atto532 (see appendix A.2 for details). Integrin is a cell membrane protein which plays a role in the attachment of cells to other cells and also of the cells to the microscope cover slip in our case. It is found in so called 'adhesion points', where the cell attaches to the glass. Figures 3.26(a) and (b) show the conventional and the GSDIM image of the stained integrin in high-glucose DMEM with addition of glucose-oxidase and catalase (DMEM, high glucose with HEPES $10 \mathrm{mM}$ and $10 \%$ (v/v) glucose-oxidase $(5 \mathrm{mg} / \mathrm{ml})$ and $2 \%(\mathrm{v} / \mathrm{v})$ catalase $(2 \mathrm{mg} / \mathrm{ml})$ ). This environment is live cell compatible as tests with U373MG cells showed. Even after $90 \mathrm{~min}-$ utes of incubation in this buffer no obvious difference in the fraction of dead cells could be seen as compared to the control sample in standard DMEM. This experiment is thus an encouraging step towards live-cell-GSDIM-imaging.

The imaging frame rate was $200 \mathrm{~Hz}$ with an illumination intensity of $115 \mathrm{~kW} / \mathrm{cm}^{2}$ at $532 \mathrm{~nm}$. Back-pumping with $375 \mathrm{~nm}$ was used after frame $\approx 10000$ with an intensity of $440 \mathrm{~W} / \mathrm{cm}^{2}$ for up to $50 \mu \mathrm{s}$ during the camera dark times. The reactivation was necessary due to the sparse staining of the integrin proteins. Due to the fact that the sample consists mainly of separated stained protein clusters of subdiffraction size, special data post-selection was applied to ensure that the recorded events stem only from single molecules as discussed in section 1.5.5. In this specific case, all events which were registered at the same position for more than three consecutive frames were discarded. If they extended over two or three frames, these events were merged and registered as one event with the position determined as the weighted average position from all frames. Furthermore all events were discarded 

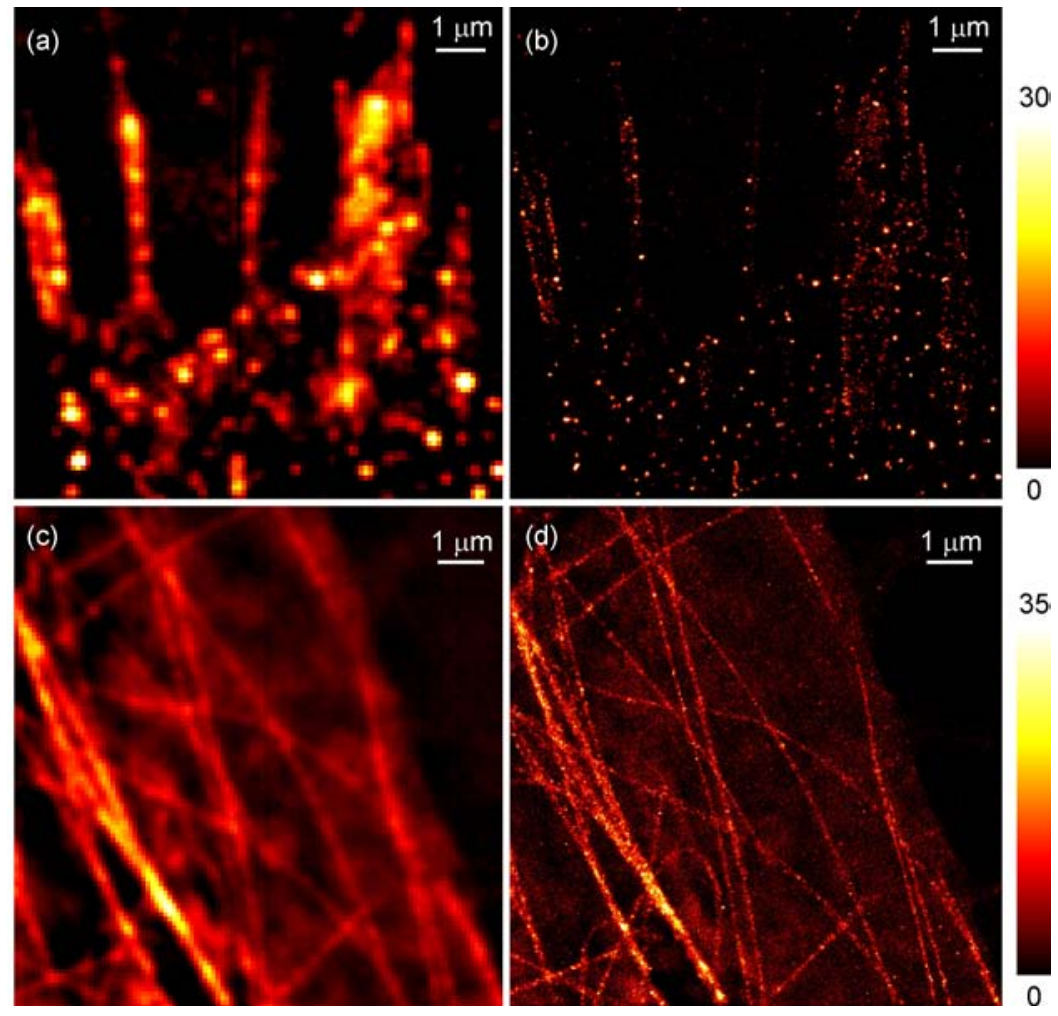

Figure 3.26: (a) Reconstructed conventional wide-field image of integrin- $\beta$ - 3 clusters in a fixed human U373 MG glioma cell stained with Atto532. (b) GSDIM-image of the same region. The image was performed in cell culture medium with addidtion of an oxygen quencher. (c) Wide-field image of microtubules of a living PtK2cell. The tubular network was stained using the fluorescent protein Citrine fused to the microtubule-associated protein Map2. The Citrine-Map2-complex was self-expressed by the cell. (d) GSDIM-image corresponding to (c). The colour bars on the right side denote the colour coding of the registered events per pixel in the GSDIM-images.

where the photon number exceeded more than 8000 in a single frame. The image consists of 130117 registered events with an average photon number of 2600 . The pixel size is $20 \mathrm{~nm}$.

Fluorescent proteins can also be efficiently transferred to a metastable dark state such as the triplet or different protonation states [97]. As can be seen in table 3.1, all fluorescent proteins tested, namely GFP, EYFP, Citrine and PhiYFP, yielded $\varepsilon<10 \%$ and a dark state lifetime $\tau>1 \mathrm{~ms}$ in aqueous media and are thus suitable for GSDIM. The conventional and GSDIM image of the microtubule network of a living PtK2 cell are illustrated in figure 3.26 (c) and (d), respectively.

31000 frames were used for the image of figure 3.26 (d), which were acquired with a rate of $200 \mathrm{~Hz}$. The illumination intensity was $2.6 \mathrm{~kW} / \mathrm{cm}^{2}$ with light of $488 \mathrm{~nm}$. The mean number of photons was 800 using a lower threshold of 80 photons. No activation light was used in this measurement. The cells were mounted in Ringer's solution $(8.6 \mathrm{~g} / 1 \mathrm{NaCl}, 0.3 \mathrm{~g} / \mathrm{KCl}$, $0.33 \mathrm{~g} / \mathrm{l} \mathrm{CaCl} 2)$ at room temperature. The staining was performed using Citrine. The cell expressed this fluorescent protein fused to the microtubule-associated protein Map2 which 
resulted in the labelling of the microtubule cytoskeleton. For further details concerning the cell preparation, plese refer to the appendix A.3.

The resolution in the GSDIM-image is $\approx 40 \mathrm{~nm}$, which was determined by measuring the FWHM of small isolated spots. The resolution is thus lower than in the case of GSDIM with organic dyes. This stems from the fact that switching to the dark state is usually faster for the fluorescent proteins than for the organic dyes, restricting the number $N$ of photons in a burst [98]. Furthermore, the average off-time of a Citrine molecule in this particular molecular construct is rather long ( $>1 \mathrm{~s})$ as shown in table 3.1. which limits the number of on-off cycles before irreversible bleaching. As a result, the contrast of the reconstructed GSDIM image is also reduced. In comparison, Rh6G molecules return faster and more often. Nevertheless, the stronger photobleaching of Citrine in the cellular environment seems not to be of fundamental nature, because the lifetime of the dark state of Citrine proteins in pure aqueous environment is much shorter $(50 \mathrm{~ms})$, implying that Citrine is generally more photostable than in the example above. While the substantial variation in lifetime can be attributed to changes in the $\mathrm{pH}$ or in the concentration of molecular oxygen, this example highlights the relevance of the molecular environment in a particular application of GSDIM.

\subsubsection{Summary}

In this section the applicability of conventional non-switchable fluorescent dyes and proteins for SMS-microscopy was shown. In previous experiments only special photoswitchable markers such as the fluorescent proteins EosFP [14], PA-GFP [16],Dronpa [23] or rsFastLime [17, 18, 80], and organic Cyanin dyes in special configurations and environments [15, 80, 95], or photoswitchable rhodamine amides [25, 26, 28] were used for SMS-type imaging.

By exploiting their long lived dark states, however, also a wide range of conventional fluorescent markers can be used for SMS-imaging, thereby rendering this method very general and not restricted to specialised markers. Given the right environment, these dark states are sufficiently long-lived to provide efficient fluorescence switch-off while still allowing the molecule to emit a large burst of photons before entering the dark state, which can be used for high-accuracy localisation.

The embedding media used here are standard media, and, in the case of DMEM buffer in addition with an oxygen quencher, even proved to be live-cell compatible. This is in contrast to experiments using switchable cyanine dyes in aqueous solutions where toxic $\beta$-mercaptoethanol had to be added [15, 80, 95].

By exploiting the metastable dark state of fluorescent proteins, it was possible to image the tubulin structure of living cells by the GSDIM approach.

GSDIM further offers the practical advantage that initially all marker molecules are in the on-state, which makes it easier to find the region of interest for imaging. Furthermore, the need of only one wavelength for both excitation and switch-off simplifies the experimental setup. 


\section{Conclusion}

Based on the general principle of switching markers between a detectable on- and a nondetectable off-state, RESOLFT- and SMS-type far field microscopy break Abbe's resolution barrier. This thesis describes the potential of photoswitchable organic markers to further enable the power of superresolution far-field fluorescence microscopy. By combining novel forms of photoswitchable organic fluorophores and novel imaging approaches, the applicability of the two microscopy concepts was extended while frequently even simplifying the experimental effort in comparison to hitherto realised implementations.

In the case of RESOLFT-microscopy, a novel approach was introduced, based on Resonance Energy Transfer between a fluorescent donor dye and a photoswitchable photochromic acceptor dye. It allowed to break the resolution barrier using light intensities of only several hundred $\mathrm{W} / \mathrm{cm}^{2}$ to a few $\mathrm{kW} / \mathrm{cm}^{2}$ as compared to several $\mathrm{GW} / \mathrm{cm}^{2}$ necessary for STEDmicroscopy, which represents the hitherto most successful RESOLFT-technique. This reduces effects of sample photodamage and makes it possible to use cost-effective low-power lasers. A resolution of $50 \mathrm{~nm}$ in one spatial direction was achieved using a mixture of the two dyes in a polymer film. A more general approach with a dyad consisting of the two dyes covalently bound, yielded a resolution of $75 \mathrm{~nm}$ in linescans. However, the performance of this new RESOLFT-approach is presently limited by the possible number of switching cycles of the dye system, which was not sufficient for two-dimensional high resolution images.

In the case of SMS-microscopy, the problem of switching fatigue is avoided, since it is sufficient if each marker is switched only once. The work on SMS-microscopy can be subdivided into three parts.

In the first part, a method was introduced which allowed for the first time the imaging of whole thick samples using SMS-high resolution microscopy. Previous SMS-experiments had been limited to thin samples. Using dyes of a novel family of photoactivatable markers, based on photoswitchable rhodamines, a two-photon activation scheme was applied, allowing for the non-invasive imaging of thick samples layer by layer, while the out-of-focus regions remained unaffected and did not produce any disturbing background light. It was shown that two-photon activation even works with continuous-wave activation light which can be supplied by compact solid state or diode lasers, rendering the system cost-effective and simple. This way of optical sectioning of a sample allows SMS-microscopy to regain one of the most important features of light microscopy, which is noninvasive imaging of thick samples, which was abandoned in the SMS-experiments performed until then.

The novel photoactivatable dye family was also used to demonstrate a new technique of multicolour-imaging for SMS-microscopy. This method exploits the single-molecule approach of SMS-microscopy also for efficient marker discrimination. In contrast to conven- 
tional multicolour imaging, also markers with significantly overlapping emission spectra can be clearly distinguished. Even fluorophores with an emission peak disparity of only $18 \mathrm{~nm}$ were separated. This allows for the simultaneous imaging of several different markers whose spectra are so closely together that they can still be excited with the same wavelength. This and the fact that only two detection channels are required simplifies the experimental setup. In the experiments described in this thesis, three different marker types were separated by using only two detection channels and one excitation laser for all dyes.

SMS-microscopy has so far had its focus on 'really' photoswitchable dyes or fluorescent proteins, whose switching process is connected to a rearrangement of atoms or chemical bonds. The number of such markers is limited. It was demonstrated in this work that the inherent electronic dark states of standard non-switchable fluorescent dyes can be exploited for the switching process in SMS-microscopy. This concept, called Ground State Depletion with Individual Marker return (GSDIM), renders a whole gamut of standard marker dyes applicable for SMS-microscopy. Since the switch-off process is induced by the fluorescence excitation light, no additional laser source is mandatory for activating and/or deactivating the molecules. By applying GSDIM also to fluorescent proteins, it was possible to image living cells.

The work of this thesis shows that photoswitchable organic fluorophores have tremendous potential in high-resolution optical microscopy. Although for their routine application in RESOLFT-microscopy, further research in terms of switching stability but also watersolubility has to be undertaken, their use in SMS-microscopy already shows their impressive capabilities. A logical further step is to combine methods such as optical sectioning with three-dimensional subdiffraction localisation and multicolour imaging to exploit the full range of possibilities of light microscopy with the SMS-concept. Additionally, special dyes sensitive to their environment can be used. In analogy to the novel photoswitchable dye family introduced in this thesis, such dyes might also be made photoswitchable and thus applicable to SMS-microscopy, giving new tools for high resolution imaging of biochemical processes. 



\section{Bibliography}

[1] Abbe, E. Beiträge zur Theorie des Mikroskops und der mikroskopischen Wahrnehmung. Arch. Mikr. Anat., 9:(1873), 413-468. vii

[2] Binnig, G., Quate, C. and Gerber, C. Atomic force microscope. Phys. Rev. Lett., 56:(1986), 930-933. viii

[3] Novotny, L. and Hecht, B., editors. Principles of Nano-Optics (Cambridge Univ. Press, Cambridge, 1993). viii

[4] Pendry, J. B. Negative Refraction Makes a Perfect Lens. Phys. Rev. Lett., 85(18):(2000), 3966-3969. viii

[5] Liu, Z., Lee, H., Xiong, Y., Sun, C. and Zhang, X. Far-Field Optical Hyperlens Magnifying Sub-Diffraction-Limited Objects. Science, 315(5819):(2007), 1686. viii

[6] Smolyaninov, I. I., Hung, Y.-J. and Davis, C. C. Magnifying Superlens in the Visible Frequency Range. Science, 315(5819):(2007), 1699-1701. viii

[7] Hell, S. W. and Stelzer, E. H. K. Fundamental improvement of resolution with a 4Pi-confocal fluorescence microscope using two-photon excitation. Opt. Commun., 93:(1992), 277-282. viii

[8] Gustafsson, M. G. L. Surpassing the lateral resolution limit by a factor of two using structured illumination microscopy. J. Microsc., 198(2):(2000), 82-87. viii, 23

[9] Hell, S. W. Improvement of lateral resolution in far-field light microscopy using twophoton excitation with offset beams. Opt. Commun., 106:(1994), 19-24. viii

[10] Hell, S. W. Strategy for far-field optical imaging and writing without diffraction limit. Phys. Lett. A, 326:(2004), 140-145. viii

[11] Hell, S. W. Far-Field Optical Nanoscopy. Science, 316(5828):(2007), 1153-1158. viii, 8, $13,14,21,22$

[12] Hell, S. W. and Wichmann, J. Breaking the diffraction resolution limit by stimulated emission: stimulated emission depletion microscopy. Opt. Lett., 19(11):(1994), 780 782. viii, 8, 24

[13] Hofmann, M., Eggeling, C., Jakobs, S. and Hell, S. W. Breaking the diffraction barrier in fluorescence microscopy at low light intensities by using reversibly photoswitchable proteins. Proc. Natl. Acad. Sci. USA, 102(49):(2005), 17565-17569. viii, ix, 8, 24, 25, 71 
[14] Betzig, E., Patterson, G., Sougrat, R., Lindwasser, O., Olenych, S., Bonifacino, J., Davidson, M., Lippincott-Schwartz, J. and Hess, H. Imaging Intracellular Fluorescent Proteins at Nanometer Resolution. Science, 313(5793):(2006), 1642-1645. viii, ix, 14 , 19, 20, 41, 43, 74, 85

[15] Rust, M. J., Bates, M. and Zhuang, X. Sub-diffraction-limit imaging by stochastic optical reconstruction microscopy (STORM). Nat. Methods, 3:(2006), 793-796. viii, ix, $14,41,74,85$

[16] Hess, S. T., Girirajan, T. P. K. and Mason, M. D. Ultra-High Resolution Imaging by Fluorescence Photoactivation Localization Microscopy. Biophys. J., 91(11):(2006), 4258-4272. viii, ix, 14, 41, 43, 45, 53, 74, 85

[17] Geisler, C., Schönle, A., von Middendorff, C., Bock, H., Eggeling, C., Egner, A. and Hell, S. W. Resolution of $\lambda / 10$ in fluorescence microscopy using fast single molecule photo-switching. Appl. Phys. A, 88(2):(2007), 223-226. viii, ix, 15, 20, 41, 42, 43, 45 , 53,85

[18] Egner, A., Geisler, C., von Middendorff, C., Bock, H., Wenzel, D., Medda, R., Andresen, M., Stiel, A., Jakobs, S., Eggeling, C., Schönle, A. and Hell, S. W. Fluorescence nanoscopy in whole cells by asnychronous localization of photoswitching emitters. Biophys. J., 93:(2007), 3285-3290. viii, ix, 15, 17, 41, 43, 45, 53, 85, 99

[19] Hell, S. W. and Kroug, M. Ground-state depletion fluorescence microscopy, a concept for breaking the diffraction resolution limit. Appl. Phys. B, 60:(1995), 495-497. viii. ix, 2, 74, 75,

[20] Bretschneider, S., Eggeling, C. and Hell, S. W. Breaking the Diffraction Barrier in Fluorescence Microscopy by Optical Shelving. Phys. Rev. Lett., 98:(2007), 218103. viii, ix, 74

[21] Bossi, M., Fölling, J., Dyba, M., Westphal, V. and Hell, S. W. Breaking the diffraction resolution barrier in far-field microscopy by molecular optical bistability. New J. Phys., 8:(2006), 275. viii, 25, 26, 27

[22] Fölling, J., Polyakova, S., Belov, V., van Blaaderen, A., Bossi, M. and Hell, S. W. Synthesis and Characterization of Photoswitchable Fluorescent Silica Nanoparticles. Small, 4(1):(2008), 134-142. viii, 32, 34

[23] Shroff, H., Galbraith, C. G., Galbraith, J. A., White, H., Gillette, J., Olenych, S., Davidson, M. W. and Betzig, E. Dual-color superresolution imaging of genetically expressed probes within individual adhesion complexes. Proc. Natl. Acad. Sci. USA, 104(51):(2007), 20308-20313. ix, 41, 45, 47, 53, 55, 83, 85

[24] Bates, M., Huang, B., Dempsey, G. and Zhuang, X. Multicolor Super-Resolution Imaging with Photo-Switchable Fluorescent Probes. Science, 317:(2007), 1749-1753. ix. 41,55 
[25] Fölling, J., Belov, V., Kunetsky, R., Medda, R., Schönle, A., Egner, A., Eggeling, C., Bossi, M. and Hell, S. W. Photochromic Rhodamines Provide Nanoscopy with Optical Sectioning. Angew. Chem. Int. Ed., 46:(2007), 6266-6270. ix, 41, 42, 74, 85, 97

[26] Fölling, J., Belov, V., Riedel, D., Schönle, A., Egner, A., Eggeling, C., Bossi, M. and Hell, S. W. Fluorescence Nanoscopy with Optical Sectioning by Two-Photon Induced Molecular Switching using Continuous-Wave Lasers. ChemPhysChem, 9:(2008), 321326. ix, 41,85

[27] Schönle, A. and Hell, S. W. Fluorescence nanoscopy goes multicolor. Nature Biotechnology, 25(11):(2007), 1234-1235. ix, 41, 55

[28] Bossi, M., Fölling, J., Belov, V. N., Boyarskiy, V. P., Medda, R., Egner, A., Eggeling, C., Schönle, A. and Hell, S. W. Multicolor Far-Field Fluorescence Nanoscopy through Isolated Detection of Distinct Molecular Species. Nano Lett., 8:(2008), 2463-2468. ix 41, 85,98

[29] Fölling, J., Bossi, M., Bock, H., Medda, R., Wurm, C. A., Hein, B., Jakobs, S., Eggeling, C. and Hell, S. W. Fluorescence Nanoscopy by Ground-State Depletion and Single-Molecule Return. Nat Methods. doi:10.1038/NMETH.1257. ix, 41, 74, 76, 78, 79, 98, 99

[30] Bretschneider, S. Ground-State-Depletion Fluorescence Microscopy. Ph.D. thesis, Georg-August-Universität Göttingen (2007). 1, 2, 74

[31] Turro, N. J. Modern Molecular Photochemistry (University Science Books, Sausalito, CA, USA, 1991). 1, 25

[32] Hecht, E. Optics (Addison Wesley, San Francisco, CA, USA, 2002). 4

[33] Goodman, J. W. Introduction to Fourier optics (Mc Graw Hill, San Francisco, CA, USA, 1968). 4

[34] Fölling, J. Bose-Einstein Josephson Tunnelling and Generation of Arbitrary Optical Potentials. Diploma thesis, Ruprecht-Karls-Universität Heidelberg (2005). 4

[35] Born, M. and Wolf, E. Principles of Optics (Cambridge University Press, Cambridge, New York, Melbourne, Madrid, Cape Town, 2002), 7th edition. 4, 7

[36] Lauterborn, W. and Kurz, T. Coherent optics: Fundamentals and Applications (Springer, Berlin, Germany, 2003). 4

[37] Meschede, D. Optics, Light and Lasers (Wiley-VCH, Weinheim, Germany, 2007). 5

[38] Minsky, M. Microscopy apparatus, US Patent 3,013,467 (1961). 5

[39] Hell, S. W., Jakobs, S. and Kastrup, L. Imaging and writing at the nanoscale with focused visible light through saturable optical transitions. Appl. Phys. A, 77:(2003), 859-860. 7, 10, 23, 24 
[40] Schwentker, M., Bock, H., Hofmann, M., Jakobs, S., Bewersdorf, J., Eggeling, C. and Hell, S. W. Wide-field subdiffraction RESOLFT microscopy using fluorescent protein photoswitching. Micr. Res. Tech., 70(3):(2007), 269-280. 7, 23

[41] Klar, T. A., Jakobs, S., Dyba, M., Egner, A. and Hell, S. W. Fluorescence microscopy with diffraction resolution limit broken by stimulated emission. Proc. Natl. Acad. Sci. USA, 97:(2000), 8206-8210. 8, 24

[42] Keller, J., Schönle, A. and Hell, S. W. Efficient fluorescence inhibition patterns for RESOLFT microscopy. Opt. Express, 15(6):(2007), 3361-3371. 8

[43] Keller, J. Optimal de-excitation patterns for RESOLFT-Microscopy. Ph.D. thesis, Ruprecht-Karls-Universität Heidelberg (2006). 8

[44] Harke, B. 3D STED Microscopy with Pulsed and Continuous Wave Lasers. Ph.D. thesis, Georg-August-Universität Göttingen (2008). 9, 24

[45] Hell, S. W., Dyba, M. and Jakobs, S. Concepts for nanoscale resolution in fluorescence microscopy. Curr. Opin. Neurobio., 14(5):(2004), 599-609. 10, 22

[46] Hell, S. W. Toward fluorescence nanoscopy. Nature Biotechnol., 21(11):(2003), 13471355. 10

[47] Westphal, V. and Hell, S. Nanoscale Resolution in the Focal Plane of an Optical Microscope. Phys. Rev. Lett., 94:(2005), 143903. 10

[48] Harke, B., Keller, J., Ullal, C. K., Westphal, V., Schoenle, A. and Hell, S. W. Resolution scaling in STED microscopy. Optics Express, 16(6):(2008), 4154-4162. 10, 13

[49] Heisenberg, W. Prinzipen der Quantentheorie (Hirzel-Verlag, Leipzig, Germany, 1930). 14, 16, 22

[50] Thompson, R., Larson, D. and Webb, W. Precise nanometer localization analysis for individual fluorescent probes. Biophys. J., 82:(2002), 2775-2783. 14, 16, 17, 18, 22

[51] Cheezum, M., Walker, W. and Guilford, W. Quantitative comparison of algorithms for tracking single fluorescent particles. Biophys. J., 81:(2001), 2378-2388. 14, 16, 18, 22

[52] von Middendorff, C. Experimental Stochastics in High Resolution Fluorescence Microscopy. Ph.D. thesis, Ruprecht-Karls-Universität Heidelberg (2008). 16, 20, 22, 54, 56,57

[53] Robbins, M. S. and Hadwen, B. J. The noise performance of electron multiplying charge-coupled devices. IEEE Trans. Electron. Dev., 50(5):(2003), 1227-1232. 17

[54] Westphal, V., Rizzoli, S. O., Lauterbach, M. A., Kamin, D., Jahn, R. and Hell, S. W. Video-Rate Far-Field Optical Nanoscopy Dissects Synaptic Vesicle Movement. Science, 320(5873):(2008), 246-249. 22 
[55] Westphal, V., Lauterbach, M. A., Di Nicola, A. and Hell, S. W. Dynamic far-field fluorescence nanoscopy. New J. Phys., 9:(2007), 435. 22

[56] Heintzmann, R., Jovin, T. M. and Cremer, C. Saturated patterned excitation microscopy - A concept for optical resolution improvement. J. Opt. Soc. Am. A: Optics and Image Science, and Vision, 19(8):(2002), 1599-1609. 23

[57] Rittweger, E., Rankin, B. R., Westphal, V. and Hell, S. W. Fluorescence depletion mechanisms in super-resolving STED microscopy. Chem. Phys. Lett., 442:(2007), 483487. 24

[58] Willig, K. I., Harke, B., Medda, R. and Hell, S. W. STED microscopy with continuous wave beams. Nature Methods, 4(11):(2007), 915-918. 24

[59] Wildanger, D., Rittweger, E., Kastrup, L. and Hell, S. W. STED microscopy with a supercontinuum laser source. Opt. Express, 16(13):(2008), 9614-9621. 24

[60] Lakowicz, J. R. Principles of fluorescence spectroscopy (Kluwer Academic/Plenum, New York, 1999). 25

[61] Valeur, B. Molecular fluorescence. Principles and applications (Wiley-VCH, Weinheim, Germany, 2001). 25

[62] Giordano, L., Jovin, T., Irie, M. and Jares-Erijman, E. Diheteroarylethenes as Thermally Stable Photoswitchable Acceptors in Photochromic Fluorescence Resonance Energy Transfer (pcFRET). J.Am.Chem.Soc., 124(25):(2002), 7481-7489. 25, 32

[63] Irie, M., Fukaminato, T., Sasaki, T., Tamai, N. and Kawai, T. A digital fluorescent molecular photoswitch. Nature, 420(6917):(2002), 759-760. 25

[64] Murase, S., Teramoto, M., Furukawa, H., Miyashita, Y. and Horie, K. Photochemically induced fluorescence control with intermolecular energy transfer from a fluorescent dye to a photochromic diarylethene in a polymer film. Macromolecules, 36(4):(2003), 964-966. 25

[65] Bossi, M., Belov, V., Polyakova, S. and Hell, S. W. Reversible red fluorescent molecular switches. Angew. Chem. Int. Ed., 45(44):(2006), 7462-7465. 32

[66] Zhao, X. J., Tapec-Dytioco, R. and Tan, W. H. Ultrasensitive DNA detection using highly fluorescent bioconjugated nanoparticles. Journal of the American Chemical Society, 125(38):(2003), 11474-11475. 33

[67] Santra, S., Zhang, P., Wang, K. M., Tapec, R. and Tan, W. H. Conjugation of biomolecules with luminophore-doped silica nanoparticles for photostable biomarkers. Analytical Chemistry, 73(20):(2001), 4988-4993. 33

[68] Selvan, S. T., Tan, T. T. and Ying, J. Y. Robust, non-cytotoxic, silica-coated CdSe quantum dots with efficient photoluminescence. Advanced Materials, 17(13):(2005), $1620-1625.33$ 
[69] Wang, L., Yang, C. Y. and Tan, W. H. Dual-luminophore-doped silica nanoparticles for multiplexed signaling. Nano Letters, 5(1):(2005), 37-43. 34

[70] Wang, L. and Tan, W. H. Multicolor FRET silica nanoparticles by single wavelength excitation. Nano Letters, 6(1):(2006), 84-88. 34

[71] Brandriss, S. and Margel, S. Synthesis and Characterization of Self-Assembled Hydrophobic Monolayer Coatings on Silica Colloids. Langmuir, 9(5):(1993), 1232-1240. 34

[72] van Blaaderen, A. and Vrij, A. Synthesis and Characterization of Colloidal Dispersions of Fluorescent, Monodisperse Silica Spheres. Langmuir, 8(12):(1992), 2921-2931. 34

[73] van Blaaderen, A., van Geest, J. and Vrij, A. Monodisperse Colloidal Silica Spheres from Tetraalkoxysilanes - Particle Formation and Growth-Mechanism. Journal of Colloid and Interface Science, 154(2):(1992), 481-501. 34

[74] Andresen, M., Stiel, A. C., Fölling, J., Wenzel, D., Schönle, A., Egner, A., Eggeling, C., Hell, S. W. and Jakobs, S. Photoswitchable fluorescent proteins enable monochromatic multilabel imaging and dual color fluorescence nanoscopy. Nat Biotechnol, 26(9):(2008), 1035-1040. 41, 70, 98

[75] Knauer, K. H. and Gleiter, R. Photochromism of Rhodamine Derivatives. Angew. Chem. Int. Ed., 16(2):(1977), 113-113. 42

[76] Willwohl, H., Wolfrum, J. and Gleiter, R. Kinetics and Mechanism of the Photochromism of N-Phenyl-Rhodaminelactame. Laser Chem., 10:(1989), 63-72. 42 , 44

[77] Feringa, B. L., editor. Molecular Switches (Wiley-VCH, 2001). 42

[78] Göppert-Mayer, M. Über Elementarakte mit zwei Quantensprüngen. Ann. Phys. (Leipzig), 9:(1931), 273-295. 43

[79] Denk, W., Strickler, J. H. and Webb, W. W. Two-photon laser scanning fluorescence microscopy. Science, 248:(1990), 73-76. 43, 44

[80] Bock, H., Geisler, C., Wurm, C. A., Jakobs, S., Schönle, A., Egner, A., Hell, S. W. and Eggeling, C. Two-color far-field fluorescence nanoscopy based on photoswitching emitters. Appl. Phys. B, 88(161-165). 45, 53, 55, 74, 85

[81] Shannon, C. E. Communication in the presence of noise. Proc. Inst. Radio. Eng., 37(1):(1949), 10-21. 47, 83

[82] Booth, M. and Hell, S. W. Continuous wave excitation two-photon fluorescence microscopy exemplified with the $647 \mathrm{~nm}$ ArKr line. J. Microsc., 190:(1998), 298-304. 50 
[83] Kao, H. P. and Verkman, A. S. Tracking of single fluorescent particles in three dimensions: Use of cylindrical optics to encode particle position. Biophys J, 67:(1994), 12911300. 54

[84] Speidel, M., Jonas, A. and Florin, E.-L. Three-dimensional tracking of fluorescent nanoparticles with subnanometer precision by use of off-focus imaging. Optics Letters, 28:(2003), 69-71. 54

[85] Toprak, E., Balci, H., Blehm, B. and Selvin, P. Three-dimensional particle tracking via bifocal imaging. Nano Letters, 7:(2007), 2043-2045. 54

[86] Juette, M., Gould, T., Lessard, M., Mlodzianoski, M., Nagpure, B., Bennett, B., Hess, S. and Bewersdorf, J. Three-dimensional sub-100 nm resolution fluorescence microscopy of thick samples. Nature Methods, 5:(2008), 527-529. 54

[87] Huang, B., Wang, W., Bates, M. and Zhuang, X. Three-Dimensional Super-Resolution Imaging by Stochastic Optical Reconstruction Microscopy. Science, 319:(2008), 810 813. 54

[88] Donnert, G., Keller, J., Wurm, C. A., Rizzoli, S. O., Westphal, V., Schönle, A., Jahn, R., Jakobs, S., Eggeling, C. and Hell, S. W. Two-Color Far-field Fluorescence Nanoscopy. Biophys. J., 92(8):(2007), L67-69L. 55

[89] Shera, E. B., Seitzinger, N. K., Davis, L. M., Keller, R. A. and Soper, S. A. Detection of single fluorescent molecules. Chem. Phys. Lett., 174(6):(1990), 553-557. 55

[90] Zander, C., Sauer, M., Drexhage, K. H., Ko, D.-S., Schulz, A., Wolfrum, J., Brand, L. and Eggeling, C. Detection and characterization of single molecules in aqueous solution. Appl. Phys. B, 63:(1996), 517-523. 55

[91] Ha, T., Enderle, T. and Chemla, D. Single molecule dynamics studied by polarization modulation. Phys. Rev. Lett., 77(19):(1996), 3979-3982. 55

[92] Ha, T., Enderle, T., Chemla, D. and Weiss, S. Dual-molecule spectroscopy: Molecular rulers for the study of biological macromolecules. IEEE J. Sel. Top. Quantum Elect., 2(4):(1996), 1115-1128. 55

[93] Fries, J. R., Brand, L. ., Eggeling, C., Köllner, M. and Seidel, C. A. M. Quantitative identification of different single-molecules by selective time-resolved confocal fluorescence spectroscopy. J. Phys. Chem. A, 102:(1998), 6601-6613. 55

[94] Neher, R. and Neher, E. Optimizing imaging parameters for the separation of multiple labels in a fluorescence image. J. Microsc., 213(1):(2004), 46-62. 66

[95] Heilemann, M., van de Linde, S., Schüttpelz, M., Kasper, R., Seefeldt, B., Mukherjee, A., Tinnefeld, P. and Sauer, M. Subdiffraction-Resolution Fluorescence Imaging with Conventional Fluorescent Probes. Angew. Chem., 47(33):(2008), 6172-6176. 74, 85 
[96] Zondervan, R., Kulzer, F., Orlinskii, S. and Orrit, M. Photoblinking of rhodamine 6G in poly(vinyl alcohol): Radical dark state formed through the triplet. J. Phys. Chem. A, 107(35):(2003), 6770-6776. 75

[97] Dickson, R. M., Cubitt, A. B., Tsien, R. Y. and Moerner, W. E. On/off blinking and switching behaviour of single molecules of green fluorescent protein. Nature, 388(6640):(1997), 355-358. 84

[98] Eggeling, C., Hilbert, M., Bock, H., Ringemann, C., Hofmann, M., Stiel, A. C., Andresen, M., Jakobs, S., Egner, A., Schönle, A. and Hell, S. W. Reversible photoswitching enables single-molecule fluorescence fluctuation spectroscopy at high molecular concentration. Micr. Res. Tech., 70:(2007), 1003-1009. 85

[99] Weber, K., Bibring, T. and Osborn, M. Specific visualization of tubulin-containing structures in tissue culture cells by immunofluorescence. Cytoplasmic microtubules, vinblastine-induced paracrystals, and mitotic figures. Exp Cell Res, 95(1):(1975), 111120. 97 


\section{A Appendix}

\section{A.1 Standard Cell Preparation}

In the following the typical preparation of cellular samples is described, as it was used in the experiments in sections 3.1.5, 3.2.4 and 3.3.3 where immunostaining was applied [25].

The mammalian PtK2 cell line was grown as described elsewhere [99]. The cells were then seeded and grown on standard glass coverslips to a confluency of about $50 \%$ and fixed with cold methanol $\left(4^{\circ} \mathrm{C}\right)$ for $4 \mathrm{~min}$, followed by incubation in blocking buffer (Phosphate Buffered Saline (PBS) containing 1\% Bovine Serum Albumin (BSA)).

Human glioma cell line U373MG was grown in RPMI Glutamax high glucose, supplemented with $10 \% \mathrm{FBS}, 100 \mathrm{U} / \mathrm{ml}$ penicillin, $100 \mu \mathrm{g} / \mathrm{ml}$ streptomycin and $1 \mathrm{mM}$ sodium pyruvate (Gibco). Cells were seeded and fixed as described above. All cells were grown at $37^{\circ} \mathrm{C}$ in a water-saturated atmosphere with $5 \% \mathrm{CO}_{2}$.

Unless otherwise described in the main text, all measurements were performed using fixed cells.

Unless otherwise mentioned, the cells were embedded in Mowiol, which is composed of 4.8 g PVA (MOWIOL, Type 4-88, Fluka (Sigma-Aldrich), St. Louis, MO, USA), 12 g glycerol, $12 \mathrm{ml}$ water, $24 \mathrm{ml}$ TRIS-buffer $\mathrm{pH} 8.4,0.2 \mathrm{M}, 0.1 \%$ (w/v) DABCO.

\section{A.2 Immunostaining of Cells}

One of the standard methods to stain specific parts of fixed cells is immunostaining. The staining is usually performed in two steps. Firstly, the structure of interest is marked with antibodies, which bind to that structure with a very high specificity. This is performed by putting a drop of blocking-solution including the antibody onto the glass coverslip on which the cells are mounted. This antibody is called 'primary antibody'. In a second step, the so called 'secondary antibody' is applied to the cell. This sort of antibody specifically recognises the primary antibody and has the actual dye coupled to it. Several dye molecules can bind to one secondary antibody and several secondary antibodies to one primary antibody, leading to a signal amplification.

Antibodies are Y-shaped proteins, the recognition site is inside the bifurcated part. The size of an antibody is roughly $7 \mathrm{~nm}$. A small structure in the nanometre range may therefore look larger due to the attached antibodies on either side.

Staining procedure in experiments of section 3.1.5 [25]: Immunostaining of microtubules of PtK2 cells was performed with anti- $\beta$-tubulin mouse IgG (Sigma) and SRA577conjugated sheep anti-mouse $\mathrm{IgG}$, as primary and secondary antibody respectively. Nuclear 
lamina of U373MG cells was stained using anti-lamin A mouse IgG (Abcam, Cambridge, UK) and SRA577-conjugated sheep anti-mouse IgG. All antibodies were diluted in blocking buffer and incubated for $1 \mathrm{~h}$ each, followed by several washes with blocking buffer.

Staining procedure in experiments of section 3.2.4 [28]: Immunostaining of microtubules of PtK2 cells was performed with anti- $\beta$-tubulin mouse IgG (Sigma) and SRA552conjugated sheep anti-mouse IgG, as primary and secondary antibody respectively. The sheep anti-mouse IgG was modified with the Traut's reagent under nitrogen, in order to transform the terminal (lysine) amino groups into thiols and then coupled with the compound SRA552-maleimide. Keratin was stained with anti-cytokeratin-widespectrum rabbit IgG (Abcam) as primary and SRA577-conjugated goat anti-rabbit IgG as secondary antibody. All antibodies were diluted in blocking buffer and incubated for $1 \mathrm{~h}$ at room temperature, followed by several washes with blocking buffer.

Staining procedure in experiments of section 3.3.3 [29]: The microtubules were stained with anti- $\beta$-tubulin mouse IgG (Sigma, St. Louis, MO, USA) and the corresponding dye conjugated to sheep anti-mouse IgG (Sigma). The peroxisomes were labelled with anti-PMP70 rabbit IgG (Abcam) and Atto532-conjugated sheep anti-rabbit IgG or Atto565-conjugated goat anti-rabbit IgG. Imaging of the immunostained cells was either performed in PVA (spincoating of 1\% PVA in PBS (pH 7.4) solution at $3000 \mathrm{rpm}$ ) or in standard aqueous imaging buffer (50 mM Tris, pH 7.5, $10 \mathrm{mM} \mathrm{NaCl}, 0.5 \mathrm{mg} / \mathrm{mL}$ glucose oxidase (G2133, Sigma), $40 \mathrm{~g} / \mathrm{mL}$ catalase (106810, Roche Applied Science) and 10\% (w/v) glucose). The images of figure 3.26(a),(b) were taken from mammalian U373 MG glioma cells. The cells were fixed for 4 min with cold methanol and immunostaining of integrin- $\beta-3$ has been performed using anti-integrin- $\beta$-3 IgG as primary and Atto532-conjugated sheep anti-mouse IgG antibody as secondary antibody. The sample was embedded in standard growing medium (DMEM, high glucose, Invitrogen, Karlsruhe, Germany) with addition of $10 \%(\mathrm{v} / \mathrm{v})$ of glucose oxidase $(5 \mathrm{mg} / \mathrm{ml})$ and $2 \%(\mathrm{v} / \mathrm{v})$ catalase $(2 \mathrm{mg} / \mathrm{ml})$. While this medium is compatible with live cell measurements, cell fixation was required to prevent clustering of integrin- $\beta-3$ due to the antibody labelling.

\section{A.3 Staining with Fluorescent Proteins}

Fluorescent proteins can be expressed by the cell itself if the according DNA sequence is added to the DNA of the cell. This can be performed such that the fluorescent protein is already attached to the protein of interest. Alternatively, the fluorescent protein can be expressed such that it is bound to another protein, which in turn binds to the structure of interest.

Sample preparation for the experiments in section 3.2.5 [74]: To label the E.coli cytoplasmic membrane with bsDronpa or Dronpa, fusion proteins were expressed consisting of the M13 bacteriophage procoat protein fused to either of these proteins. M13 is integrated into the cytoplasmic membrane. The coding sequences of bsDronpa or Dronpa were PCR amplified and inserted into a modified pET28 expression vector harbouring a M13-GFP fusion28, thereby replacing the GFP coding sequence. Thin cryosections were prepared and 
mounted as described previously [18].

Sample preparation for the experiments involving fluorescent proteins in section 3.3 [29]: Protein purification: Plasmids coding for the fluorescent proteins GFP (pRSETA-GFP), EYFP (pRSETA-EYFP), Citrine3 (pQE31-Citrine) and PhiYFP (pQE31-PhiYFP; Evrogen, Moscow, Russia) were transformed into Escherichia coli BL21CodonPlus RIL (Stratagene, La Jolla, CA, USA). For protein expression, cells carrying the plasmid were grown to an OD600 of 0.6 in LB medium containing $100 \mu \mathrm{g} / \mathrm{ml}$ ampicillin and were induced with $1 \mathrm{mM}$ isopropyl-1-thio-L-D-galactopyranoside. For ensemble measurements, $100 \mu \mathrm{l}$ of Poly-LLysine solution (Sigma-Aldrich, Steinheim, Germany) was dried on a microscope cover slip. After short rinsing with desalinated water $50 \mu \mathrm{l}$ of the protein solution (in PBS buffer, $\mathrm{pH}$ 7.4) was added. After one hour non-ligated proteins were shortly rinsed off with water, $10 \mu \mathrm{l}$ of PBS buffer ( $\mathrm{pH}$ 7.4) was added, and the sample was put on a sample holder and sealed with silicone.

Plasmid construction for cellular staining: Standard methods were used for cloning. To tag the tubulin-binding protein Map2 at its $\mathrm{N}$-terminus with Citrine, the expression plasmid pSEMS-Citrine-Map2 was constructed by Gateway vector conversion (Invitrogen, Carlsbad, CA, USA) from the donor vector pDONR223-Map24 and the empty destination vector pSEMS-Citrine, a modified version of pSEMS-SNAP26m-Gateway (Covalys Biosciences, Witterswil, Switzerland). Cell culture, transfection and mounting of the cells. For transfection, PtK2 cells were grown over night on glass cover slips. After reaching $\approx 80 \%$ confluency, the plasmid pSEMS-Citrine-Map2 was introduced (Nanofectin kit, PAA, Pasching, Austria). One day after transfection cells expressing Citrine-Map2 were visible. Typically they were imaged within the following day. For microscopy, cells were rinsed with cell culture medium without phenol red. Finally the living cells were mounted with prewarmed Ringer's solution ( $8.6 \mathrm{~g} / \mathrm{l} \mathrm{NaCl}, 0.3 \mathrm{~g} / \mathrm{l} \mathrm{KCl}, 0.33 \mathrm{~g} / \mathrm{l} \mathrm{CaCl} 2)$ and sealed with silicone. 


\section{B List of Publications}

Parts of this thesis have been published as follows:

1. Bossi, M., Fölling, J., Dyba, M., Westphal, V. and Hell, S. W. Breaking the diffraction resolution barrier in far-field microscopy by molecular optical bistability. New J. Phys., 8:(2006), 275.

2. Fölling, J., Polyakova, S., Belov, V., van Blaaderen, A., Bossi, M. and Hell, S. W. Synthesis and Characterization of Photoswitchable Fluorescent Silica Nanoparticles. Small, 4(1):(2008), 134142.

3. Fölling, J., Belov, V., Kunetsky, R., Medda, R., Schonle, A., Egner, A., Eggeling, C., Bossi, M. and Hell, S. W. Photochromic Rhodamines Provide Nanoscopy with Optical Sectioning. Angew. Chem. Int. Ed., 46:(2007), 62666270.

4. Fölling, J., Belov, V., Riedel, D., Schönle, A., Egner, A., Eggeling, C., Bossi, M. and Hell, S. W. Fluorescence Nanoscopy with Optical Sectioning by Two-Photon Induced Molecular Switching using Continuous-Wave Lasers. ChemPhysChem, 9:(2008), 321326.

5. Bossi*, M., Fölling*, J., Belov, V. N., Boyarskiy, V. P., Medda, R., Egner, A., Eggeling, C., Schönle, A. and Hell, S. W. Multicolor Far-Field Fluorescence Nanoscopy through Isolated Detection of Distinct Molecular Species. Nano Lett., 8:(2008), 24632468.

*These authors contributed equally to this work.

6. Andresen, M., Stiel, A. C., Fölling, J., Wenzel, D., Schönle, A., Egner, A., Eggeling, C., Hell, S. W. and Jakobs, S. Photoswitchable fluorescent proteins enable monochromatic multilabel imaging and dual color fluorescence nanoscopy. Nat Biotechnol, 26(9):(2008), 10351040.

7. Fölling*, J., Bossi*, M., Bock, H., Medda, R., Wurm, C. A., Hein, B., Jakobs, S., Eggeling, C. and Hell, S. W. Fluorescence Nanoscopy by Ground-State Depletion and Single-Molecule Return. Nat Methods. doi:10.1038/NMETH.1257.

*These authors contributed equally to this work. 


\section{Acknowledgement}

I would like to thank all the people who have contributed to the success of this thesis.

- Stefan W. Hell for the opportunity to work in his group. He provided an outstanding scientific environment and continuously supported this work with new ideas and good advice.

- Tim Salditt for his interest in this project and for his willingness to be one of the referees of this thesis.

- Mariano Bossi for his excellent mentoring during the whole thesis. His ideas contributed a lot to the success of this work. It was a great pleasure to work with him. He also prepared all silica nano-particles used in this thesis and performed several of the measurements concerning the dye characterisation whose results are shown in chapter 2.

- Christian Eggeling for his equally good supervision especially in the second half of my $\mathrm{PhD}$ project. He provided a lot of great ideas and was a great source of help and support in every aspect and whenever needed.

- Volker Westphal for his assistance and advice particularly during the first part of my project. It was very enjoyable and also extremely informative to work with him.

- Vladimir Belov and his 'Organic Chemistry Group' including Svetlana Polyakova, Vadim Boyarskiy, Roman Kunetsky and Sergey Yan for doing a great job synthesising the switchable rhodamine amides and the Rh-AA-DAE.

- Andreas Schönle for his ideas and support especially in the multicolour-SMS-project. Furthermore for providing the data-acquisition-software 'Imspector', for his contributions to the SMS-data analysis software and for proof-reading parts of the manuscript.

- Alexander Egner for his contribution to the SMS-data analysis software and a for lot of good advice and helpful discussions especially during the SMS-projects.

- Rebecca Medda for a lot of discussions concerning biology and much more, for preparing bright biological samples and teaching me how to be a biologist myself.

- Hannes Bock for his collaboration in the GSD project.

- Claas v. Middendorf for help and assistance during several projects and for his contribution to the SMS-data analysis. 
- Jan Keller for manifold valuable discussions and for proof-reading parts of the manuscript.

- Claudia Geisler for a lot of helpful discussions.

- Birka Hein for her contributions and ideas concerning the GSDIM-project.

- Christian Wurm for answering a lot of questions concerning biology and for his contributions to the GSDIM-project.

- Stefan Jacobs for help and advice with many biological questions.

- Martin Andresen for the collaboration in the bsDronpa-project.

- Steffen Sahl for help in the GSDIM project.

- Daniel Neumann for helping out with cells and Jessica Schilde for help with cell staining with fluorescent proteins.

- Jaydev Jethwa for always offering assistance. Be it problems with lasers or whatever, he always had a solution. Furthermore I would like to thank him for proof-reading parts of the manuscript.

- Tanja Gilat, Sylvia Löbermann and Ellen Rothermel for their support.

- Rainer Pick and Donald Ouw for their assistance.

- Harald Meyer for technical support.

- Marco Roose for helping with a lot of computer problems.

- The mechanical, optics and electronic workshops for their help. They did great jobs and were really flexible and helpful if something needed do be done a little quicker than usual...

- Sarah Aschemann, Anja Dietrich and Josephine Stadler for their friendly help with various kinds of organisational issues.

- Simon Fölling, Hannes Horst, Marcel Lauterbach, Brian Rankin and Chaytania Ullal for proof-reading (parts of) the manuscript.

- Dominik Wildanger for help with $\mathrm{LT}_{\mathrm{E}} \mathrm{X}$.

- My office and laboratory colleagues Katrin Willig, Birka Hein, Annedore Punge, Benjamin Harke, Marcel Lauterbach and Roman Schmidt for a lot of fruitful, funny and serious scientific and non-scientific discussions. It was a great time!

- Many thanks to all other colleagues for their help and 'simply' for the very nice time I had the pleasure to enjoy with them.

I thank my parents for their unconditional support at all times. 


\section{Lebenslauf}

\section{Persönliche Daten}

Name, Vorname

Fölling, Jonas

Geburtsdatum

05.03.1979

Geburtsort

Familienstand

Oldenburg (Oldb)

Nationalität

ledig

deutsch

\section{Schulausbildung}

$\begin{array}{ll}1985-1989 & \text { Grundschule, Oldenburg } \\ 1989-1990 & \text { Orientierungsstufe Eversten, Oldenburg } \\ 1990-1998 & \text { Albrecht-Altdorfer-Gymnasium, Regensburg }\end{array}$

\section{Zivildienst}

1998-1999

Klinik für Anästhesiologie, Universitätsklinikum Regensburg

\section{Studium}

$1999-2001$

Grundstudium der Physik, Universität Konstanz

$2001-2005$

Haupstudium der Physik, Universität Heidelberg

2002- 2003

Auslandsstudium an der Université d'Ottawa/University of Ottawa, Ottawa, Kanada

seit 2005

Promotion am Max-Planck-Institut für Biophysikalische

Chemie, Abteilung Nanobiophotonik

Göttingen, den 22.10.2008 Jonas Fölling 